\title{
Structure Theorems for Partially Asynchronous Iterations of a Nonnegative Matrix with Random Delays *
}

\author{
Reza Gharavi \\ School of Electrical Engineering \\ Cornell University \\ Ithaca, NY 14853 \\ AND \\ Venkat Anantharam ${ }^{\dagger}$ \\ Department of Electrical Engineering \\ 8 Computer Sciences \\ University of California \\ Berkeley, CA 94720 \\ ananth@vyasa.eecs. berkeley.edu
}

\begin{abstract}
We consider partially asynchronous parallel iteration of a fixed nonnegative matrix with stationary ergodic interprocessor communication delays. We study the iteration via a random graph describing the interprocessor influences. Our major result is an invariant description of the rates of convergence of arbitrary sequences of individual processor-time values. In the course of proving this result a number of other invariant properties of the convergence of the iteration are described. The convergence rates that appear in our results are Lyapunov exponents of certain random matrix products derived from the original matrix and the statistics of the delays.
\end{abstract}

${ }^{*}$ Research supported by the National Science Foundation, IBM, the AT \&T Foundation, and by BellCore Inc.

${ }^{\dagger}$ Address all correspondence to the second author 


\section{Introduction}

In this paper, we are interested in studying the asymptotic behavior of a linear iteration in a partially asynchronous parallel computation environment. Such systems arise in a wide range of applications. We will carry out a detailed analysis of the convergence properties of the computation when the interprocessor delays are modelled as a stationary ergodic process.

We first motivate the problem that will be studied. Consider solving a system of linear equations $M y=c$, where $M$ is an $p \times p$ matrix, $c$ is a $p$ vector, and $y$ is to be determined. Even though direct methods, such as Gaussian elimination, yield exact solutions after a finite number of steps, it may be advantageous to use iterative methods when $M$ is large and sparse. This is because iterative methods can yield very accurate results after a relatively small number of steps and they have smaller memory requirements. Large and sparse $M$ arise often in areas such as discretization of partial linear differential equations, network theory, power distribution systems, structural analysis, and graph theory; see

Section 2.5 of [1], [4], and [5] for detailed examples drawn from such areas. To use the iterative method one first finds matrices $A$ and $D$ such that the equation $y=A y+D c$ has a fixed point solving $M y=c$. The fixed point can then be determined by iteration. See [3] and [5] for a discussion of how to obtain suitable $A$ and $D$ from $M$.

As another important example, consider evaluating the value function associated to any fixed policy in the dynamic programming approach to Markov decision problems. In discounted dynamic programming, for instance, this reduces to finding the fixed point of the equation $y=c+\alpha P y$. where $\alpha \in(0,1)$ is a discount factor, $c$ is the cost vector associated with some stationary policy, and $P$ is the transition probability matrix associated with this policy, see [9], for instance, for a discussion of the theory of discounted dynamic programming. Again, in most applications, $P$ is large and sparse, making iterative approaches to finding the fixed point advantageous over direct methods.

The sequence of iterates of the fixed point equation $y=A y+D c$ converges to the fixed point if and only if the sequence of iterates $y(n+1)=A y(n)$ converges to zero. Therefore, the convergence characteristics of iterations of the type $y(n+1)=A y(n)$ are central to the quantifying the efficiency of the iterative approach to such problems.

We may now define the problem of interest in this paper. In carrying out the iteration in a parallel processing environment, there are $p$ processors, each responsible for comput- 
ing a component of a vector $x(n)$. Due to communication delays among the processors, or between each processor and a shared memory, at each time $n$, processor $i$ may compute $x_{i}(n)$ using possibly outdated values, that is,

$$
x_{i}(n)=\sum_{j=1}^{p} a_{i j} x_{j}\left(n-d_{i j}(n)\right) .
$$

where $d_{i j}(n)>0$ is the communication delay from processor $j$ to processor $i$ at processor $i$ at time $n$. We say then that the computation proceeds asynchronously. Asynchronous implementations of iterations have certain advantages over synchronous ones. For one, since fast processors don't have to wait for messages from every processor, they can execute more iterations, and so a potential speed advantage is gained over synchronous algorithms. See section 6.3.5 of [1] for a discussion of this issue. Secondly, synchronization overhead is reduced. Finally, particularly in distributed systems, such implementations are natural, and sometimes even necessary. See [1] for a thorough discussion of such algorithms.

In this study, we impose two conditions on the delays. Firstly, we assume all the delays to be bounded by some integer $B>0$. Using the terminology of [1], we say that our system is partially asynchronous. Secondly, we assume that the statistics of the delays can be described by a stationary process. By the ergodic decomposition theorem (see section 7.4 of [7]), we lose little generality by assuming ergodicity, so we do so. We impose no other a priori condition on the delays; in particular, messages between two processors may overtake each other and $d_{i i}$ may be bigger than one, which may occur, for example, when processors share a common memory. On the other hand, if it is known that restrictions such as $d_{i i} \equiv 1$ hold, this can be incorporated by appropriate choice of the statistics of the delays in our model.

In this paper we are interested in analyzing the convergence characteristics of the recursion (1) in a partially asynchronous computational environment with stationary and ergodic delays. For most of our results we will assume that the matrix $A$ and the initial conditions are nonnegative.

We now summarize the results of this paper. The results are naturally broken up into two parts, and the paper is organized along these lines. In Part I, we carry out a detailed analysis of the structure of the interprocessor influences, without explicitly introducing the issue of convergence rates of an iteration. In Part II, we use the results proved in Part 
I to prove results about such convergence rates.

We first describe the results of Part I. By a scenario, say $\omega$, we mean a particular realization of the interprocessor delays $\left(d_{i j}(n), n \in \mathbf{Z}\right)$. We assume that this process is stationary and ergodic, with

$$
1 \leq d_{i j}(n) \leq B \text { for all } 1 \leq i, j \leq p \text { and all } n \in \mathbf{Z}
$$

The evolution of the computation is described on a computation graph which has one node $(q, n)$ for each processor $q$ at each time $n \in \mathbf{Z}$, by assigning real numbers to each node $(q, n)$ with $n \geq-B+1$. The values at nodes $(q, n)$ with $n \in\{-B+1, \ldots, 0\}$ are the initial conditions, and the corresponding nodes in the computation graph are called the base. To each node $(q, n)$ with $n \geq 1$ there is associated a subset of the base which consists of those initial conditions that determine the value of that node (value of the datum at that processor at that time). This is called the color of the node. The set of all colors that show up infinitely often is called the spectrum, $\mathcal{S}(\omega)$, of the scenario $\omega$. The first main result is that in an almost sure shift invariant (a.s.s.i.) subset of scenarios the cardinality of the spectrum is a fixed non-random constant, call it $\sigma$.

To any scenario $\omega$ we can associate a shifted scenario $\theta \omega$, which is the one corresponding to the delays $\left\{d_{i j}(n+1), n \in \mathbf{Z}\right\}$. Another way to think of this is to focus on the nodes of the computation graph from times $-B+2$ onwards but with the times renumbered by decreasing them by 1 . Thus each node $u$ gets a color in the shifted scenario. The second main result is that, for $n$ large enough, in an a.s.s.i. subset of scenarios, the color of a node in any shifted scenario depends only on its color in the original scenario. We describe this correspondence explicitly, in the form of a rule which yields $\mathcal{S}\left(\theta^{n} \omega\right)$ from $\mathcal{S}(\omega)$ for all $n \in \mathbf{Z}$. Let $\theta^{n} C_{i}$ denote the unique color in $\mathcal{S}\left(\theta^{n} \omega\right)$ corresponding to $C_{i} \in \mathcal{S}(\omega)$, $1 \leq i \leq \sigma$.

The colors of the spectrum are partially ordered by inclusion. Choose an arbitrary numbering of the $\sigma$ colors of the spectrum as $\left\{C_{1}(\omega), \ldots, C_{\sigma}(\omega)\right\}$. We then get a partial order on $\{1, \ldots, \sigma\}$ induced by the inclusion partial order on colors. Consider the equivalence class of this ordering under reindexing of the colors. The third main result is that, in an a.s.s.i. subset of scenarios, the equivalence class of this partial order is a nonrandom constant.

We identify levels of this inclusion partial order inductively, saying that a color is at level 1 if it contains no other color, and that it is at level $i$ if it is not of level $i-1$ or 
less and any color that it properly contains is of level $i-1$ or less. Let $\mathcal{S}_{i}(\omega)$ denote the subset of colors at level $i$ in scenario $\omega$. A consequence of the previous result is that the number of levels is a non-random constant, call it $\rho$. We define the notion of a pure color of level $i$ as one that is not contained in the union of the colors of level $i$ or less other than itself. The fourth main result is the following equivalent characterization of purity : a color is pure precisely if it is not the union of the colors of level $i-1$ or less that it properly contains. This is a rather remarkable and unexpected result. We note that if in the partial order on $\{1, \ldots, \sigma\}$ corresponding to the inclusion partial order on colors of the spectrum we mark which colors are pure, then the equivalence class under reindexing of the colors of this marked partial order is constant in an a.s.s.i. subset of scenarios.

The color of a node is defined only for nodes with positive time index. Further, even though the number of colors that appear infinitely often is a.s. a nonrandom constant, as shown in the first main result, there can be other colors that show up finitely often. To avoid this problem a subset of the base, called the extended color, is associated to every node in a shift invariant way; this matches the color for all nodes with sufficiently large time indices. Using this concept, it is shown that in describing how the value of a node is determined during a computation it suffices, for each color of the spectrum, to focus on a special subset of nodes, called the filament of nodes associated to that color. Thus, convergence properties of the iteration will be determined by the convergence properties of the values of nodes in the filaments. This result, which is the fifth main result of Part I, involves the construction of certain naturally defined stationary point processes associated to the computation graph. This completes the summary of the results of Part I.

We next describe the results of Part II. For our main results we will assume that the entries of the matrix being iterated, as well as the initial conditions, are all nonnegative. Since the assumed bound on the delays makes $x(n)$ depend linearly on the components of $x(n-1), \ldots, x(n-B)$, it is possible to construct a $B p$-dimensional system with $y(n)=$ $\left[x^{T}(n) x^{T}(n-1) \ldots x^{T}(n-B+1)\right]^{T} \in \mathbf{R}^{B p}$, and a sequence of $B p \times B p$ matrices $A(n)$ satisfying $A(n+1) y(n)=y(n+1)$ for $n \in \mathbf{Z}$. The statistical assumptions on the delays make the sequence of matrices $\{A(n), n \in \mathbf{Z}\}$ stationary and ergodic. In addition, since $A(1)$ attains at most $B^{p^{2}}$ different values and its elements are either zero, one, or those of A, $E(\max (0, \log \|A(1)\|))<\infty$. It is therefore natural to apply Oseledec's multiplicative ergodic theorem (OMET) to our system (see [2],[7]). 
Theorem 1 (Oseledec's Multiplicative Ergodic Theorem (OMET) ) If $\{A(n), n \in \mathbf{Z}\}$ is a stationary ergodic sequence of $B p \times B p$ matrices and $E(\max (0, \log \|A(1)\|))<\infty$, then there exists a nonrandom integer $s \leq B p$ with nonrandom constants $-\infty \leq \lambda_{s}<\ldots<$ $\lambda_{1}<\infty$, and nonrandom nonzero integers $\delta_{1}, \ldots, \delta_{s}$ satisfying $\sum_{k=1}^{s} \delta_{k}=B p$, such that the following hold almost surely:

1. The random sets

$$
V_{k}=\left\{y \in \mathbf{R}^{B p}: \lim _{n \rightarrow \infty} n^{-1} \log \|A(n) \cdots A(1) y\| \leq \lambda_{k}\right\}
$$

are subspaces and if $\theta$ is the shift on the probability space for which $A(n, \theta \omega)=A(n+1, \omega)$, then $A(1, \omega) V_{k}(\omega) \subseteq V_{k}(\theta \omega)$,

2. $\operatorname{dim} V_{k}=\sum_{i \geq k} \delta_{i}$,

3. If $y \in V_{k} \backslash V_{k+1}$, with $V_{s+1}$ defined as $\{0\}$, then

$$
\lim _{n \rightarrow \infty} n^{-1} \log \|A(n) \cdots A(1) y\|=\lambda_{k}
$$

for $k=1, \ldots, s$.

Definition 1 (Lyapunov exponents) For each $k=1, \ldots, s$, the constant $\lambda_{k}$ is called a Lyapunov exponent, $\delta_{k}$ is called the multiplicity of exponent $\lambda_{k}$, and $V_{k}$ is called the Oseledec subspace associated with $\lambda_{k}$.

Thus, depending on the initial condition, the computation can converge at one of only $s$ convergence rates $\lambda_{1}, \ldots, \lambda_{s}$. In particular, $x(n)$ converges to zero for all initial conditions if and only if $\lambda_{1}<0$. (For a sufficient condition on $A$ for this to occur, see [3]).

The collection of nodes

$$
\{(q, m): 1 \leq q \leq p, n-B+1 \leq m \leq n\}
$$

is called the $n$-th $B$-block. The vector $y(n)$ lists the values at the nodes of this $B$-block. Consider now the rate of growth of the norm of the value of $B$-blocks,

$$
\lim _{n \rightarrow \infty} n^{-1} \log \|y(n)\|
$$

for a given nonnegative initial condition $y(0)$. In view of the nonnegativity assumptions, this is the maximum of the rates of growths for the basic initial conditions (which consist 
of setting just one coordinate of the base to 1 and the others to 0 ) corresponding to the nonzero coordinates of $y(0)$. The first main result of Part II may be described as follows: Choose an arbitrary numbering of the colors of the spectrum as $\left(C_{1}(\omega), \ldots, C_{\sigma}(\omega)\right)$. For any $b \in\{0,1\}^{\sigma}$, there is an associated subset of colors, consisting of those $C_{i}(\omega)$ with $b_{i}=1$. Then to any $b \in\{0,1\}^{\sigma}$ we can associate a mark which is either the symbol $\phi$ or one of the Lyapunov exponents $\lambda_{1}, \ldots, \lambda_{s}$. The meaning of the mark $\phi$ is that for no $n \in \mathbf{Z}$ is it true that $\bigcap_{i: b_{i}=1} \theta^{n} C_{i} \backslash \bigcup_{i: b_{i}=0} \theta^{n} C_{i}$ is nonempty. The meaning of mark $\lambda_{i}$ is that there are infinitely many $n \in \mathbf{Z}$ such that $\bigcap_{i: b_{i}=1} \theta^{n} C_{i} \backslash \bigcup_{i: b_{i}=0} \theta^{n} C_{i}$ is nonempty and for any basic initial condition lying in this set, the rate of growth of $B$-blocks for scenario $\theta^{n} \omega$ is the Lyapunov exponent $\lambda_{i}$. Further the equivalence class of this marking upto reindexing of the colors is a non-random constant in an a.s.s.i. subset of scenarios.

It is possible to decompose the matrix that describes the evolution of $B$-blocks in a way compatible with the hierarchical structure of the colors partially ordered by inclusion. This decomposition is block lower triangular in nature. Each of the diagonal blocks of this decomposition, of which there are $\rho+1$, gives a stationary ergodic sequence of random matrices, with associated set of Lyapunov exponents $\Lambda_{i}$. Let us now consider a sequence of nodes with time indices increasing to infinity and an initial condition $y(0)$. We are interested in the rate of growth of the value of the nodes. As before, by virtue of the nonnegativity assumption, it suffices to consider basic initial conditions. Further, by virtue of the finiteness of the number of possible colors, it suffices to consider subsequences of same colored nodes going to infinity. Let us focus on the basic initial condition associated to node $u$ in the base and on a sequence of $C$ colored nodes going to infinity, where $C \in \mathcal{S}(\omega)$.

The second main result of Part II is also the main result of the paper, and may be described as follows : in an a.s.s.i. subset of scenarios, it is possible to assign to every pure color $C \in \mathcal{S}(\omega)$ a number $\lambda_{C}>-\infty$. If $C \in \mathcal{S}_{i}(\omega)$, then $\lambda_{C} \in \Lambda_{i}$. Further, for any color $C \in \mathcal{S}(\omega)$, there is a rate of growth of the processor time values along any sequence of $C$ colored nodes when the basic initial condition corresponds to $u \in W$, call this $\lambda(C, u)$, which does not depend what sequence of $C$ colored nodes we go to infinity along. $\lambda(C, u)$ can be inductively described as follows: If $u \notin C$ the rate of growth is $-\infty$ (this is obvious). If $C$ is at level 1 and $u \in C$, then the rate of growth is precisely $\lambda_{C}$ for all $u \in C$. (Note that every color at level 1 is pure). If $C$ is at level $i$ and is not pure 
and $u \in C, \lambda(C, u)$ is the maximum of $\lambda\left(C_{j}, u\right)$ over all colors $C_{j}$ that are contained in $C$ (hence necessarily at lower levels) and which contain $u$. If $C$ is at level $i$ and is pure, and if $u \in C$, then $\lambda(C, u)$ is the maximum of $\lambda_{C}$ and the maximum of $\lambda\left(C_{j}, u\right)$ over all colors $C_{j}$ that are contained in $C$ (hence necessarily at lower levels) and which contain $u$. If in the partial order on $\{1, \ldots, \sigma\}$ corresponding to the inclusion partial order on colors of the spectrum we mark each pure color $C$ by its corresponding number $\lambda_{C}$, then the equivalence class under reindexing of the colors of this marked partial order is constant in an a.s.s.i. subset of scenarios.

Finally, in Part II, we also are able to use the analysis of Part I to prove some results in the case where the nonnegativity restrictions on the matrix and on the initial conditions are lifted. These results are much weaker than the results proven under the nonnegativity assumptions. In the scalar case, we prove that the Oseledec subspaces are just direct sums of subspaces associated to the colors. In the matrix case, we prove that the colors give an invariant refinement of the Oseledec subspaces. This completes the summary of the results in Part II.

We now proceed to a formal development of the above results. 


\section{PART I}

\section{Cardinality of the Spectrum}

\subsection{Problem Setup}

Let $(\Omega, \mathcal{F}, P)$ be a probability space and $\theta: \Omega \rightarrow \Omega$ be an invertible transformation on $\Omega$. We assume $(P, \theta)$ to be stationary, that is, $P\left(\theta^{-1} F\right)=P(F)$ for all $F \in \mathcal{F}$. We also assume $(P, \theta)$ to be ergodic, that is, $P(F)$ is either zero or one for all $F \in \mathcal{F}$ such that $\theta F=F$. By the ergodic decomposition theorem (see section 7.4 of [7]), we lose little generality by this additional ergodicity assumption.

We characterize the delays by the measurable mapping $d: \mathbf{Z} \times \Omega \rightarrow\{1, \ldots, B\}^{p \times p}$, which is compatible with $\theta$, that is, $d(n, \theta \omega)=d(n+1, \omega)$ for all $\omega \in \Omega$ and $n \in \mathbf{Z}$. Given a scenario $\omega \in \Omega$ and some initial condition $\left\{x_{i}(n) ;-B+1 \leq n \leq 0,1 \leq i \leq p\right\}$, the value of processor $i$ at time $n, x_{i}(n, \omega) \in \mathbf{R}$, evolves according to the relation

$$
x_{i}(n, \omega)=\sum_{j=1}^{p} a_{i j} x_{j}\left(n-d_{i j}(n, \omega)\right), \quad 1 \leq i \leq p, n \geq 1,
$$

where $x_{i}(n, \omega)=x_{i}(n)$ for $-B+1 \leq n \leq 0,1 \leq i \leq p$.

Remark 1 Except in the last section of the paper, Section 9, we will assume that both $A$ and the initial conditions are nonnegative.

\subsection{Computation graph}

The interprocessor influences are described by a computation graph.

Definition 2 (Computation graph) For each scenario $\omega \in \Omega$, define a directed graph $G(\omega)=(U, E(\omega))$ called the computation graph by

$$
\begin{aligned}
U & =\{1, \ldots, p\} \times \mathbf{Z} \\
E(\omega) & =\left\{<\left(q_{1}, n_{1}\right),\left(q_{2}, n_{2}\right)>: a_{q_{2}, q_{1}} \neq 0, n_{1}=n_{2}-d_{q_{2}, q_{1}}\left(n_{2}, \omega\right), n_{2}>0\right\} .
\end{aligned}
$$

Remark 2 To simplify notation, we use $u, v, w, u_{0}, v_{0}, w_{0}$ etc. as typical member of $U$. For $u=(q, n)$, define $T(u)=n$ and $u+i=(q, n+i)$ We then say that node $u$ is shifted by $i$ time units. 
From compatibility of the delay process with $\theta$ we see that, for all $\omega \in \Omega$,

$$
E\left(\theta^{n} \omega\right)=\{<u-n, v-n>:\langle u, v>\in E(\omega)\}, \quad n \in \mathbf{Z} .
$$

Definition 3 (Base) Let $W=\{u \in U:-B+1 \leq T(u) \leq 0\}$. We call $W$ the base of the computation graph.

To set up the computation, initial conditions are specified by assigning values to the nodes in the base.

\subsection{History, Color, and Spectrum}

Definition 4 (History) For any scenario $\omega \in \Omega$, for a node $w \in U$, its history $H(w, \omega)$ is defined to be the set of those nodes $v \in U$ such that there exists a nontrivial directed path from $v$ to $w$ in $G(\omega)$.

Note that, for all $\omega \in \Omega$,

$$
H\left(w, \theta^{n} \omega\right)=H(w+n, \omega)-n, \quad n \in \mathbf{Z} .
$$

Definition 5 (Color) For any scenario $\omega \in \Omega$, for a node $w=(q, n)$ with $n \geq 1$, its color $C(w, \omega)$ is defined as

$$
C(w, \omega)=\{u \in W:(u, v) \in E(\omega) \text { for some } v \in H(w, \omega) \cup\{w\} \text { with } T(v) \geq 1\}
$$

Given an initial condition (an assignment of values to the nodes in the base) the values at the nodes in the color of a node determine the value at that node. Note that any nodes in $W$ that lead to $w$ only through other nodes in $W$ are not included in $C(w, \omega)$. Indeed, their values will not affect the value of $w$.

Definition 6 (Spectrum) For any scenario $\omega \in \Omega$, define a collection of subsets of the base $\mathcal{S}(\omega)$ by

$$
\begin{array}{r}
\mathcal{S}(\omega)=\left\{C \in 2^{W}: \forall n_{0} \geq 1, \exists w \in U\right. \text { such that } \\
\left.T(w) \geq n_{0} \text { and } C(w, \omega)=C\right\} .
\end{array}
$$

$\mathcal{S}(\omega)$ is called the spectrum of the computation graph $G(\omega)$. In words, $C \in \mathcal{S}(\omega)$ if and only if there are infinitely many $C$-colored nodes in $G(\omega)$. 


\subsection{An illustrative example}

We now provide a simple example to better illustrate these concepts. Let $A$ be a $2 \times 2$ matrix with no zero elements. Let $B=2$ and $\Omega=\left\{\omega_{0}, \omega_{1}\right\}$ with $P\left(\omega_{i}\right)=.5$ and $\theta \omega_{i}=\omega_{(i+1) \bmod 2}$. Clearly, $(P, \theta)$ is stationary and ergodic. Let $d\left(n, \omega_{i}\right)=d\left(n \bmod 2, \omega_{i}\right)$ (i.e. the delays are periodic with period 2), with

$$
d\left(1, \omega_{0}\right)=\left(\begin{array}{ll}
1 & 1 \\
1 & 2
\end{array}\right) \quad \text { and } \quad d\left(2, \omega_{0}\right)=\left(\begin{array}{ll}
2 & 2 \\
2 & 2
\end{array}\right) .
$$

To make $d$ compatible with $\theta$, we let $d\left(n, \omega_{1}\right)=d\left((n+1) \bmod 2, \omega_{0}\right)$.

After constructing $G\left(\omega_{0}\right)$ it is easy to show that, for example,

$$
\begin{aligned}
& H\left((2,1), \omega_{0}\right) \cap W=\{(1,0),(2,-1)\} \\
& H\left((1,2), \omega_{0}\right) \cap W=\{(1,0),(2,0)\} \\
& H\left((2,3), \omega_{0}\right) \cap W=\{(1,0),(2,0),(2,-1)\},
\end{aligned}
$$

and also

$$
\begin{aligned}
& C\left((2,1), \omega_{0}\right)=\{(1,0),(2,-1)\} \\
& C\left((1,2), \omega_{0}\right)=C_{1} \\
& C\left((2,3), \omega_{0}\right)=C_{2}
\end{aligned}
$$

where $C_{1}=\{(1,0),(2,0)\}$ and $C_{2}=\{(1,0),(2,0),(2,-1)\}$.

In a similar way, we can show that the colors of $(1,3),(1,2)$, and $(2,2)$ in $G\left(\omega_{0}\right)$ are all $C_{1}$. Then using the periodicity of the delays we can show that

$$
\{(1,2+2 n),(2,2+2 n),(1,1+2 n): n \geq 0\}
$$

is the set of all nodes with color $C_{1}$, while

$$
\{(2,1+2 n): n \geq 1\}
$$

is the set of all nodes with color $C_{2}$. Since only a finite number of nodes with positive time index are not included in the two subsets above, we get $\mathcal{S}\left(\omega_{0}\right)=\left\{C_{1}, C_{2}\right\}$.

Now consider $G\left(\omega_{1}\right)$. It is easy to show that, for example,

$$
\left.H\left((2,2), \omega_{1}\right) \cap W=\{(2,0),(1,-1),(2,-1))\right\} .
$$


As was done for $G\left(\omega_{0}\right)$, we can show that $\mathcal{S}\left(\omega_{1}\right)=\left\{C_{1}^{\prime}, C_{2}^{\prime}\right\}$ with $C_{1}^{\prime}=\{(1,-1),(2,-1)\}$ and $C_{2}^{\prime}=\{(2,0),(1,-1),(2,-1)\}$. Similarly, we can also show that

$$
\{(1,2+2 n),(1,1+2 n),(2,1+2 n): n \geq 0\}
$$

is the set of nodes with color $C_{1}^{\prime}$ in scenario $\omega_{1}$ while

$$
\{(2,2+2 n): n \geq 0\}
$$

is the set of nodes with color $C_{2}^{\prime}$ in this scenario.

\subsection{The spectrum of the time shifted graph}

Note that the spectrum $\mathcal{S}(\omega)$ is defined for each individual scenario $\omega \in \Omega$, and there is no obvious correspondence among colors of $\mathcal{S}(\omega)$ for different $\omega$ 's. In particular, $C \in \mathcal{S}(\omega)$ is not a priori a random function (unless $|\mathcal{S}(\omega)| \equiv 1$ ) as there is no canonical way of assigning $C$ to $\omega$. However, in an a.s.s.i. subset of $\Omega$, there are certain important invariant properties of $\mathcal{S}(\omega)$.

We first show that for all scenarios $\omega \in \Omega$, there are at least as many colors in the spectrum of $G(\theta \omega)$ as there are in the spectrum of $G(\omega)$. We then use ergodicity to show that the cardinality of the spectrum is constant in an a.s.s.i. subset of scenarios.

Definition 7 For any scenario $\omega \in \Omega$, for a color $C \in \mathcal{S}(\omega)$, define

$$
\mathcal{B}_{C}(\omega)=\left\{C^{\prime} \in 2^{W}: \mid\left\{w: C(w, \omega)=C \text { and } C(w-1, \theta \omega)=C^{\prime}\right\} \mid=\infty\right\}
$$

Remark 3 Note that since there are only $2^{B p}$ distinct subsets of $W$ and there are infinitely many nodes with color $C$, we must have $\mathcal{B}_{C}(\omega) \neq \emptyset$ for each $C \in \mathcal{S}(\omega)$.

Proposition 1 For all scenarios $\omega \in \Omega,|\mathcal{S}(\omega)| \leq|\mathcal{S}(\theta \omega)|$.

Proof : We will first show that

$$
\mathcal{S}(\theta \omega)=\bigcup_{C \in \mathcal{S}(\omega)} \mathcal{B}_{C}(\omega)
$$

Let $C^{\prime} \in \mathcal{B}_{C}(\omega)$. By definition, $C^{\prime}$ is the color of infinitely many nodes under scenario $\theta \omega$, so $C^{\prime} \in \mathcal{S}(\theta \omega)$. Conversely, let $C^{\prime} \in \mathcal{S}(\theta \omega)$. Then for all $n>1$, there exists a $w$ with 
$T(w) \geq n$ and $C(w, \theta \omega)=C^{\prime}$. Clearly, there is a $C$ such that for infinitely many such nodes $w$, we have in addition that $C(w+1, \omega)=C$. Then by definition, $C \in \mathcal{S}(\omega)$ and $C^{\prime} \in \mathcal{B}_{C}(\omega)$.

We will next show that

$$
C_{1}, C_{2} \in \mathcal{S}(\omega) \quad \text { with } \quad C_{1} \neq C_{2} \quad \text { implies } \quad \mathcal{B}_{C_{1}}(\omega) \cap \mathcal{B}_{C_{2}}(\omega)=\emptyset
$$

which, in conjunction with Remark 3 and equation (8) completes the proof.

Let $C_{1}, C_{2} \in \mathcal{S}(\omega)$ with $C_{1} \neq C_{2}$. With no loss of generality we can pick $u \in C_{1} \backslash C_{2}$. Let $C^{\prime} \in \mathcal{B}_{C_{1}}(\omega)$. We will show that $C^{\prime} \notin \mathcal{B}_{C_{2}}(\omega)$. We separate the cases $u-1 \in C^{\prime}$ and $u-1 \notin C^{\prime}$.

Case 1: $u-1 \in C^{\prime}$. Pick $w$ such that $C(w-1, \theta \omega)=C^{\prime}$. By definition, there are infinitely such nodes as $C^{\prime} \in \mathcal{B}_{C_{1}}(\omega)$. Since $u-1 \in C^{\prime}=C(w-1, \theta \omega) \subseteq H(w-1, \theta \omega)$, we get $u \in H(w, \omega)$. Further, there is some node $v$ with $T(v) \geq 1,<u-1, v>\in E(\theta \omega)$, and $v \in H(w-1, \theta \omega) \cup\{w-1\}$, by the definition of color in equation (7). This implies that $\langle u, v+1>\in E(\omega), T(v+1) \geq 1$ and $v+1 \in H(w, \omega) \cup\{w\}$, so that, once again by the definition of color, we have $u \in C(w, \omega)$. However, since $u \notin C_{2}$, it follows that $C(w, \omega) \neq C_{2}$. Therefore, for any $w, C(w-1, \theta \omega)=C^{\prime}$ implies $C(w, \omega) \neq C_{2}$. Hence $C^{\prime} \notin \mathcal{B}_{C_{2}}(\omega)$.

Case 2: $u-1 \notin C^{\prime}$. Pick $w$ such that $C(w, \omega)=C_{1}$ and $C(w-1, \theta \omega)=C^{\prime}$. Since there are infinitely many such $w$ 's we further impose $T(w)>B$. Since $u \in C_{1}$ and $C(w, \omega)=C_{1}$, we can find $v \in H(w, \omega)$ with $T(v) \geq 1$ such that $\langle u, v>\in E(\omega)$, by the definition of color. Here we observe that such a node $v$ cannot be $w$ itself, as $T(w)>B$ and the timewise length of any edge can be no bigger than $B$. Since $u-1 \in H(w-1, \theta \omega)$, we must have $T(v)=1$ for any such $v$, because, if not, we would have $u-1 \in C(w-1, \theta \omega)=C^{\prime}$, contradicting the hypothesis.

Now $v-1 \in H(w-1, \theta \omega), C(w-1, \theta \omega)=C^{\prime}$, and $T(v-1)=0$, implies $v-1 \in C^{\prime}$. Suppose it were true that $C^{\prime} \in \mathcal{B}_{C_{2}}(\omega)$. Then we could pick $w_{0}$ such that $C\left(w_{0}, \omega\right)=C_{2}$ and $C\left(w_{0}-1, \theta \omega\right)=C^{\prime}$. Since $v-1 \in C^{\prime}$, we get $v-1 \in H\left(w_{0}-1, \theta \omega\right)$, and so $v \in H\left(w_{0}, \omega\right)$. But recalling that $\langle u, v\rangle \in E(\omega)$, we get $u \in H\left(w_{0}, \omega\right)$. Further, because $T(v) \geq 1$ and $v \in H\left(w_{0}, \omega\right)$, we get $u \in C\left(w_{0}, \omega\right)=C_{2}$, by the definition of color. This contradicts the hypothesis that $u \notin C_{2}$, completing the proof.

As a consequence of Proposition 1 and ergodicity we have the following: 
Theorem 2 There is a nonrandom constant $\sigma \leq 2^{B p}$ such that for an a.s.s.i. subset of scenarios we have $|\mathcal{S}(\omega)|=\sigma$.

Proof : Let $\Omega_{(m)}=\{\omega:|\mathcal{S}(\omega)| \geq m\}$. Since $\Omega_{(1)}=\Omega, \Omega_{\left(2^{B p}+1\right)}=\emptyset$ and $\Omega_{(m)} \subseteq \Omega_{(m-1)}$, there must exist a $\sigma \leq 2^{B p}$ such that $P\left(\Omega_{(\sigma+1)}\right)=0$ but $P\left(\Omega_{(\sigma)}\right)>0$.

On the other hand Proposition 1 implies that $\theta \Omega_{(m)} \subseteq \Omega_{(m)}$ for all $m$, which in turn by ergodicity implies that $P\left(\Omega_{(m)}\right)$ is either zero or one. Therefore, $P\left(\Omega_{(\sigma+1)}\right)=0$ and $P\left(\Omega_{(\sigma)}\right)=1$. By intersecting $\Omega_{\sigma}$ with all shifted copies of itself we get an a.s.s.i. subset of scenarios on which $|\mathcal{S}(\omega)|=\sigma$.

Remark 4 In the example of Subsection 2.4, we have $\sigma=2$.

Remark 5 Let $\Omega_{0}$ denote an a.s.s.i. subset of scenarios on which Theorem 2 holds. At any stage of the paper we will implicitly assume that all scenarios of interest belong to an a.s.s.i. subset that verifies all of the results proved up to that stage. For notational convenience we will write $\Omega_{0}$ for this subset of scenarios. Thus $\Omega_{0}$ varies as we go along. $\diamond$

\section{The spectrum of the time shifted scenario}

\subsection{The filament of a color of the spectrum}

Definition 8 (Filament) For any scenario $\omega \in \Omega$, for a color $C \in \mathcal{S}(\omega)$, its filament $F_{C}(\omega)$ is defined to be the set of all nodes that are in the history of infinitely many nodes of $\operatorname{color} C$.

Remark 6 In the example of subsection 2.4 we have

$$
\begin{aligned}
& F_{C_{1}}\left(\omega_{0}\right)=\{(1,2+2 n),(2,2+2 n): n \in \mathbf{Z}\} \\
& F_{C_{2}}\left(\omega_{0}\right)=\{(1,2+2 n),(2,2+2 n),(2,1+2 n): n \in \mathbf{Z}\},
\end{aligned}
$$

and

$$
\begin{aligned}
& F_{C_{1}^{\prime}}\left(\omega_{1}\right)=\{(1,1+2 n),(2,1+2 n): n \in \mathbf{Z}\} \\
& F_{C_{2}^{\prime}}\left(\omega_{1}\right)=\{(2,2+2 n),(1,1+2 n),(2,1+2 n): n \in \mathbf{Z}\} .
\end{aligned}
$$




\subsection{Basic properties of filaments}

The following definition was introduced earlier in equation (3).

Definition 9 (B-block) For any $n \in \mathbf{Z}$, the collection of nodes $W+n$ is called the $n$-th B-block.

Lemma 1 For any scenario $\omega \in \Omega$, for any color $C \in \mathcal{S}(\omega)$, the following hold :

(1) $F_{C}(\omega)$ contains a node in every B-block. In particular, $\left|F_{C}(\omega)\right|=\infty$.

(2) For all $u \in F_{C}(\omega)$, there exists some $v \in F_{C}(\omega)$ such that $\langle u, v\rangle \in E(\omega)$.

(3) For all $v \in F_{C}(\omega)$, if $\left\langle u, v>\in E(\omega)\right.$ then $u \in F_{C}(\omega)$.

\section{Proof :}

To prove (1), note that since the time-wise length of each edge is bounded by $B$, for any $w \in U, H(w, \omega)$ must contain at least one node in every $B$-block. Fix a $B$-block $W+n$. We know that there are infinitely many $C$-colored nodes and that the history of each of them contains at least one node in $W+n$. But there are only $B p$ nodes in $W+n$, and so at least one node in $W+n$ must be in the history of infinitely many $C$-colored nodes, that is, it must be in $F_{C}(\omega)$.

To prove $(2)$, let $u \in F_{C}(\omega)$. Since $u$ is in the history of infinitely many $C$ colored nodes and $u$ has only finitely many outgoing edges in $E(\omega)$, for some node $v$ such that $\langle u, v>\in E(\omega), v$ must be in the history of infinitely many $C$-colored nodes, i.e. $v \in$ $F_{C}(\omega)$.

To prove (3) note that $v \in F_{C}(\omega)$ means $v$ is in the history of infinitely many $C$ colored nodes. But, since $\langle u, v\rangle \in E(\omega), u$ is also in the history of these nodes. Thus $u \in F_{C}(\omega)$.

A color $C \in \mathcal{S}(\omega)$ can be characterized in terms of its filament $F_{C}(\omega)$ as follows.

Lemma 2 For any scenario $\omega \in \Omega$, for any color $C \in \mathcal{S}(\omega)$, we have :

$$
C=\left\{u \in W \text { : there exists } v \text { with } T(v) \geq 1, v \in F_{C}(\omega) \text {, and }<u, v>\in E(\omega)\right\} \text {. }
$$

In particular

$$
C \subseteq F_{C}(\omega) \cap W .
$$




\section{Proof :}

Let $u \in C$. Then $u \in W$. Further, for every $C$-colored node $w$, there is a node $v$ with $T(v) \geq 1$ such that $v \in H(w, \omega) \cup\{w\}$ and $\langle u, v\rangle \in E(\omega)$, by the definition of color. Since there are infinitely many $C$-colored nodes and only finitely many outgoing edges from $u$, some such $v$ must be in $F_{C}(\omega)$. This shows that the LHS of equation (10) is contained in its RHS.

Conversely, let $u$ be a node that verifies the conditions of the RHS of equation (10) and let $v \in F_{C}(\omega)$ with $T(v) \geq 1$ and $\langle u, v\rangle \in E(\omega)$ be as in that condition. Let $w$ having $C(w, \omega)=C$ be such that $v \in H(w, \omega)$ (infinitely many such $w$ exist). Then we have $u \in H(w, \omega) \cap W$, and we also note that such a verifies the conditions of the RHS of equation (7) in the definition of the color of $w$, which we know is $C$, so $u \in C$. This completes the proof of equation (10).

Finally, by Lemma 1, part (3), the existence of $v$ in $F_{C}(\omega)$ such that $\langle u, v\rangle \in E(\omega)$ implies $u \in F_{C}(\omega)$. This verifies equation (11).

\subsection{Time shifting of the individual colors}

In Theorem 2, we have shown that the number of colors in the spectrum is a nonrandom constant $\sigma$ in an a.s.s.i. subset of scenarios $\Omega_{0}$. There is a simple prescription to determine $\mathcal{S}(\theta \omega)$ for $\omega \in \Omega_{0}$. It involves using the computation graph $G(\omega)$ to construct a subset $\theta C$ of the base for each color $C \in \mathcal{S}(\omega)$, after which $\mathcal{S}(\theta \omega)$ is seen to consist precisely of the sets $\{\theta C: C \in \mathcal{S}(\omega)\}$.

Definition 10 ( $\theta C$ ) For any scenario $\omega \in \Omega$, for any color $C \in \mathcal{S}(\omega)$, define a set of nodes $\theta C$ by the rule:

$$
\theta C=\left\{u \in W+1:\left\langle u, v>\in E(\omega) \text { for some } v \in F_{C}(\omega) \text { with } T(v)>1\right\}-1\right. \text {. }
$$

Note that $\theta C$ is a subset of the base $W$.

Proposition 2 Let $\omega \in \Omega_{0}$. For any color $C \in \mathcal{S}(\omega)$, we have $\mathcal{B}_{C}(\omega)=\{\theta C\}$.

Proof : From Proposition 1 we know that for $\omega \in \Omega_{0}, \mathcal{B}_{C}(\omega)$ has cardinality 1, i.e. there is one and only one subset of the base, call it $C_{0}$, such that, for infinitely many nodes $w \in U$, we have $C(w, \omega)=C$ and $C(w-1, \theta \omega)=C_{0}$. With this notation, $\mathcal{B}_{C}(\omega)=\left\{C_{0}\right\}$. We will show that $C_{0}=\theta C$, where $\theta C$ is defined in equation (12). 
Let $u-1 \in \theta C$. Let $v \in F_{C}(\omega)$ with $T(v)>1$ and $\langle u, v>\in E(\omega)$ be as in the RHS of equation (12). Since $v \in F_{C}(\omega)$, for infinitely many nodes $w \in U$ we have $C(w, \omega)=C$ and $v \in H(w, \omega)$. But since $\langle u, v>\in E(\omega)$, we have $u \in H(w, \omega)$ for all such $w$. Hence $u-1 \in H(w-1, \theta \omega)$ for all such $w$. Since also $T(v-1) \geq 1$ and $v-1 \in H(w-1, \theta \omega)$ for all such $w$ we have, by the definition of color, that $u-1 \in C(w-1, \theta \omega)$ for all such $w$. Since there are infinitely many such $w$, we conclude that $C(w-1, \theta \omega)=C_{0}$ for all but finitely many such $w$. Hence $u-1 \in C_{0}$.

Conversely, let $u \in C_{0}$. For infinitely many nodes $w \in U$, we have $C(w+1, \omega)=C$ and $C(w, \theta \omega)=C_{0}$, and for each such $w$ there is some $v$ with $T(v) \geq 1,<u, v>\in E(\theta \omega)$ and $v \in H(w, \theta \omega) \cup\{w\}$, by the definition of color. Since there are only finitely many choices for such $v$, some such $v$ must be in $H(w, \theta \omega)$ for infinitely many such $w$. For such a node $v$, we have $u+1 \in W+1,<u+1, v+1>\in E(\omega), T(v+1)>1$, and $v+1 \in F_{C}(\omega)$. By the definition of $\theta C$ in equation (12), this means $u \in \theta C$.

Proposition 2 describes how $\mathcal{S}(\theta \omega)$ can be determined from $\mathcal{S}(\omega)$ for $\omega \in \Omega_{0}$. Going the other way is even easier, and works for all $\omega \in \Omega$ and for all colors (not just the ones in the spectrum).

Proposition 3 For $\omega \in \Omega$ and $u \in U$ with $T(u) \geq 1$, let $C(u, \omega)=C$. Then $C(u+$ $\left.1, \theta^{-1} \omega\right)$ can be determined from $C$.

Proof : This is a direct consequence of the definition of color. If $C(w, \omega)=V \subseteq W$, with $V=V^{0} \cup V^{1}$ where $V^{0}=\{u \in V: T(u)=0\}$ and $V^{1}=\{u \in V:-B+1 \leq T(u) \leq-1\}$, then $C\left(w+1, \theta^{-1} \omega\right)=\left(V^{1}+1\right) \cup\left(V^{2}+1\right)$ where $V^{2}=\{u \in U:\langle u, v>\in E(\omega)$ for some $v \in$ $\left.V^{0}\right\}$.

The following two simple observations are useful at several points later in the paper.

Lemma 3 Let $\omega \in \Omega$ and let $C \in \mathcal{S}(\omega)$. If $u \in W$ and $u-1 \in \theta C$, then $u \in C$.

Proof : By equation (12), for some $v \in F_{C}(\omega)$ with $T(v)>1$ we have $\langle u, v>\in E(\omega)$. By equation (10) we have $u \in C$.

Lemma 4 Let $\omega \in \Omega$ and let $C \in \mathcal{S}(\omega)$. If $u \in C$ and $u-1 \notin \theta C$, then

$$
\left\{v: T(v) \geq 1, v \in F_{C}(\omega),<u, v>\in E(\omega)\right\}
$$

is nonempty and $v-1 \in \theta C$ for every $v$ in this set (so that, in particular, $T(v)=1$ for every $v$ in this set). 
Proof : Since $u \in C$, by equation (10) the set of equation (13) is nonempty. If $T(v)>1$ for some $v$ in this set, then, by equation (12) we have $u-1 \in \theta C$, contradicting the hypothesis, so we must have $T(v)=1$ for every $v$ in this set. By Lemma 1, part (2), for any such $v$, there is some $w \in F_{C}(\omega)$ such that $\langle v, w\rangle \in E(\omega)$ and since $T(v)=1$ we must have $T(w)>1$ for any such $w$. By equation (12) this means $v-1 \in \theta C$.

\subsection{Shifted colors}

Let $\omega \in \Omega_{0}$, and let $C \in \mathcal{S}(\omega)$. Then, by Proposition 2, $\theta C \in \mathcal{S}(\theta \omega)$. But, since also $\theta \omega \in \Omega_{0}$, we can define $\theta^{2} C=\theta(\theta C)$. We then have $\theta^{2} C \in \mathcal{S}\left(\theta^{2} \omega\right)$, so that, since $\theta^{2} \omega \in \Omega_{0}$, we can define $\theta^{3} C=\theta\left(\theta^{2} C\right)$. Proceeding in this way, $\theta^{n} C$ is well defined for all $n \geq 0$, for all $C \in \mathcal{S}(\omega)$, for all $\omega \in \Omega_{0}$.

For $\omega \in \Omega_{0}$, our definition of $\theta C$ from $C$ gives an isomorphism from $\mathcal{S}\left(\theta^{-1} \omega\right)$ to $\mathcal{S}(\omega)$, because $\theta^{-1} \omega \in \Omega_{0}$. So there exists an inverse $\theta^{-1}$ from $\mathcal{S}(\omega)$ into $\mathcal{S}\left(\theta^{-1} \omega\right)$ : this is

the unique function, denoted $\theta^{-1}$, from $\mathcal{S}(\omega)$ into $\mathcal{S}\left(\theta^{-1} \omega\right)$ such that, if $C \in \mathcal{S}(\omega)$ and $C^{\prime} \in \mathcal{S}\left(\theta^{-1} \omega\right)$, then $\theta^{-1} \theta C^{\prime}=C^{\prime}$ and $\theta \theta^{-1} C=C$.

Likewise, for $\omega \in \Omega_{0}$, for any $n>0, \theta^{n}$ is an isomorphism from $\mathcal{S}\left(\theta^{-n} \omega\right)$ to $\mathcal{S}(\omega)$, because $\theta^{-n} \omega \in \Omega_{0}$. This has an inverse, denoted $\theta^{-n}$, mapping $\mathcal{S}(\omega)$ to $\mathcal{S}\left(\theta^{-n} \omega\right)$. We denote by $\theta^{-n} C$ the image in $\mathcal{S}\left(\theta^{-n} \omega\right)$ of $C \in \mathcal{S}(\omega)$ under this map. Note that, if $C \in \mathcal{S}(\omega)$ and $C^{\prime} \in \mathcal{S}\left(\theta^{-n} \omega\right)$, then $\theta^{-n} \theta^{n} C^{\prime}=C^{\prime}$ and $\theta^{n} \theta^{-n} C=C$.

So now, for any $\omega \in \Omega_{0}$ and any $n \in \mathbf{Z}$, a map $\theta^{n}: \mathcal{S}(\omega) \mapsto \mathcal{S}\left(\theta^{n} \omega\right)$ is well defined. We claim that for all $m, l \in \mathbf{Z}$, all $C \in \mathcal{S}(\omega)$ and all $\omega \in \Omega_{0}$, we have

$$
\theta^{m+l} C=\theta^{m}\left(\theta^{l} C\right)
$$

and further, if $\mathcal{S}(\omega)=\left\{C_{1}, \ldots, C_{\sigma}\right\}$, then $\mathcal{S}\left(\theta^{n} \omega\right)=\left\{\theta^{n} C_{1}, \ldots, \theta^{n} C_{\sigma}\right\}$, for all $n \in \mathbf{Z}$. The verification of this is straightforward, and is left to the reader.

Next we note that, for $\omega \in \Omega_{0}$, we have

$$
\mid\{w: C(w, \omega)=C, \text { and } C(w-1, \theta \omega) \neq \theta C\} \mid<\infty
$$

This is just a restatement of Proposition 2.

Since also $\theta \omega \in \Omega_{0}$, we also have

$$
\mid\left\{w: C(w, \theta \omega)=\theta C \text { and } C\left(w-1, \theta^{2} \omega\right) \neq \theta^{2} C\right\} \mid<\infty
$$


Combining this with equation (14), we get

$$
\mid\left\{w: C(w, \omega)=C \text { and } C\left(w-2, \theta^{2} \omega\right) \neq \theta^{2} C\right\} \mid<\infty .
$$

Continuing this process, we learn that, for all $\omega \in \Omega_{0}$, for each $C \in \mathcal{S}(\omega)$,

$$
\mid\left\{w: C(w, \omega)=C \text { and } C\left(w-n, \theta^{n} \omega\right) \neq \theta^{n} C\right\} \mid<\infty, \quad \text { for any } n \geq 0 \text {. }
$$

For a scenario $\omega \in \Omega_{0}$, for any color $C \in \mathcal{S}(\omega)$, we claim that

$$
\mid\left\{w: C(w, \omega)=C, \text { and } C\left(w+1, \theta^{-1} \omega\right) \neq \theta^{-1} C\right\} \mid=0 .
$$

This can be seen from Proposition 3. Suppose $\omega \in \Omega_{0}$, and $C \in \mathcal{S}(\omega)$. Then there are infinitely many nodes $w$ with $C(w, \omega)=C$, and, by Proposition 3, for each of these $C\left(w+1, \theta^{-1} \omega\right)$ must be the same. By Proposition 2, this can only hold if $C\left(w+1, \theta^{-1} \omega\right)=$ $\theta^{-1} C$ for all such nodes.

Since also $\theta^{-1} \omega \in \Omega_{0}$, we also have

$$
\mid\left\{w: C\left(w, \theta^{-1} \omega\right)=\theta^{-1} C \text { and } C\left(w+1, \theta^{-2} \omega\right) \neq \theta^{-2} C\right\} \mid=0 .
$$

Combining this with equation (18), we get

$$
\mid\left\{w: C(w, \omega)=C \text { and } C\left(w+2, \theta^{-2} \omega\right) \neq \theta^{-2} C\right\} \mid=0
$$

Continuing this process, we learn that, for all $\omega \in \Omega_{0}$, for each $C \in \mathcal{S}(\omega)$, and for any $n>0$

$$
\mid\left\{w: C(w, \omega)=C, \text { and } C\left(w+n, \theta^{-n} \omega\right) \neq \theta^{-n} C\right\} \mid=0 .
$$

In conjunction with equation (17) we may now write, for a scenario $\omega \in \Omega_{0}$, and a color $C \in \mathcal{S}(\omega)$ :

$$
\mid\left\{w: C(w, \omega)=C \text { and } C\left(w-n, \theta^{n} \omega\right) \neq \theta^{n} C\right\} \mid<\infty, \quad \text { for any } n \in \mathbf{Z} \text {. }
$$

\subsection{Shifted filaments and Shifted colors}

We next characterize the behavior of filaments under the shift in the a.s.s.i. subset of scenarios $\Omega_{0}$.

Lemma 5 Let $\omega \in \Omega_{0}$ and let $C \in \mathcal{S}(\omega)$. Then, for all $n \in \mathbf{Z}$, we have :

$$
F_{\theta^{n} C}\left(\theta^{n} \omega\right)=F_{C}(\omega)-n
$$


Proof : Let $n \in \mathbf{Z}$ be fixed.

We first show that the RHS of equation (23) is contained in its LHS. Let $u \in F_{C}(\omega)$. Then, for infinitely many nodes $w \in U$, with $C(w, \omega)=C$, we have $u \in H(w, \omega)$. By equation (22), for all but finitely many of these nodes, we have $C\left(w-n, \theta^{n} \omega\right)=\theta^{n} C$. We also have $u-n \in H\left(w-n, \theta^{n} \omega\right)$ for all such nodes. Hence $u-n \in F_{\theta^{n} C}\left(\theta^{n} \omega\right)$.

The proof that the LHS of equation (23) is contained in its RHS is identical.

The relation between the shifted colors and shifted filaments in the a.s.s.i. subset of scenarios $\Omega_{0}$ is made clear in the following result.

Lemma 6 Let $\omega \in \Omega_{0}$ and let $C \in \mathcal{S}(\omega)$. Then, for any $n \in \mathbf{Z}$, we have :

$$
\theta^{n} C=\left\{u \in W+n:\left\langle u, v>\in E(\omega) \text { for some } v \in F_{C}(\omega) \text { with } T(v)>n\right\}-n .\right.
$$

In particular

$$
\theta^{n} C \subseteq F_{C}(\omega) \cap(W+n)-n
$$

Proof : Let $u \in \theta^{n} C$. Then by equation (10) there is some node $v \in U$ with $T(v) \geq 1$, $v \in F_{\theta^{n} C}\left(\theta^{n} \omega\right)$, and $\langle u, v\rangle \in E\left(\theta^{n} \omega\right)$. By Lemma 5 , we have $v+n \in F_{C}(\omega)$ for this $v$. Then $u+n \in W+n,\left\langle u+n, v+n>\in E(\omega), v+n \in F_{C}(\omega)\right.$, and $T(v+n)>n$, all of which verifies that the LHS of equation (24) is contained in its RHS.

Conversely, let $u \in W+n$, and let $v$ be a node with $T(v)>n,\langle u, v>\in E(\omega)$, and $v \in F_{C}(\omega)$. By Lemma 5, we have $v-n \in F_{\theta^{n} C}\left(\theta^{n} \omega\right)$. Also, $T(v-n)>0$, $<u-n, v-n>\in E\left(\theta^{n} \omega\right)$, and $u-n \in W$, so that, by equation (10), we have $u-n \in \theta^{n} C$. This verifies that the RHS of equation (24) is contained in its LHS.

Since we have shown that any $u \in \theta^{n} C$ verifies the conditions in the brackets on the RHS of equation (24), in particular any such $u$ is in the history of a node in $F_{C}(\omega)$ and, by Lemma 1, part (3), it must be itself in $F_{C}(\omega)$. From this equation (25) follows.

Remark 7 In the example of subsection 2.4, we have $\mathcal{S}\left(\omega_{0}\right)=\left\{C_{1}, C_{2}\right\}$ and $\mathcal{S}\left(\theta \omega_{0}\right)=$ $\left\{C_{1}^{\prime}, C_{2}^{\prime}\right\}$ Here $\theta C_{1}=C_{1}^{\prime}$ and $\theta C_{2}=C_{2}^{\prime}$. In Remark 6 we explicitly identified the filaments $F_{C_{1}}\left(\omega_{0}\right), F_{C_{2}}\left(\omega_{0}\right), F_{C_{1}^{\prime}}\left(\theta \omega_{0}\right)$, and $F_{C_{2}^{\prime}}\left(\theta \omega_{0}\right)$. It is straightforward to verify the truth of equations (10), (23), and (24) from the expressions in Remark 6. 


\section{The Inclusion Structure of the Spectrum}

In Proposition 2 we have demonstrated an explicit one to one correspondence between the spectrum $\mathcal{S}(\omega)$ and the spectrum $\mathcal{S}(\theta \omega)$ in the a.s.s.i. subset of scenarios $\Omega_{0}$. Here we study this correspondence in more detail.

\subsection{Intersections and inclusions of shifted colors}

We may ask to what extent the set theoretic relations among the colors are maintained under the shift. For example, if two colors $C_{1}$ and $C_{2}$ in the spectrum of a scenario $w \in \Omega_{0}$ intersect, do their shifted versions $\theta C_{1}$ and $\theta C_{2}$ in the spectrum of $\theta \omega$ also intersect? The next proposition demonstrates that several such relationships are maintained under the shift, in an a.s.s.i. subset of scenarios.

Proposition 4 For an a.s.s.i. subset of of scenarios, for any pair of distinct colors $C_{1}$ and $C_{2}$, the following statements are true:

(1). If $C_{1} \cap C_{2}=\emptyset$, then $\theta C_{1} \cap \theta C_{2}=\emptyset$.

(2). If $C_{1} \cap C_{2} \neq \emptyset$, then $\theta C_{1} \cap \theta C_{2} \neq \emptyset$.

(3). If $C_{1} \cap C_{2}=\emptyset$, then $F_{C_{1}} \cap F_{C_{2}}=\emptyset$.

(4). If $C_{1} \cap C_{2} \neq \emptyset$, then $\left|F_{C_{1}} \cap F_{C_{2}}\right|=\infty$ and there exists $C_{3} \in \mathcal{S}(\omega)$ such that $C_{3} \subseteq C_{1} \cap C_{2}$.

(5). If $C_{1} \nsubseteq C_{2}$, then $\theta C_{1} \nsubseteq \theta C_{2}$.

(6). If $C_{1} \subseteq C_{2}$, then $\theta C_{1} \subseteq \theta C_{2}$.

(7). If $C_{1} \subseteq C_{2}$, then $F_{C_{1}} \subseteq F_{C_{2}}$.

Proof : Let $\omega \in \Omega_{0}$. To prove (1), let $C_{1}, C_{2} \in \mathcal{S}(\omega)$ and assume that $\theta C_{1} \cap \theta C_{2} \neq$ $\emptyset$. Let $v \in \theta C_{1} \cap \theta C_{2}$. Suppose first that $T(v+1) \leq 0$. Then there exists $w_{1}$ with $T\left(w_{1}\right)>1, w_{1} \in F_{C_{1}}(\omega),<v+1, w_{1}>\in E(\omega)$ (resp. $w_{2}$ with $T\left(w_{2}\right)>1, w_{2} \in F_{C_{2}}(\omega)$, $\left.<v+1, w_{2}>\in E(\omega)\right)$ which verifies the conditions in the brackets on the RHS of equation (24) because $v \in \theta C_{1}$ (resp. $v \in \theta C_{2}$ ). Then, from equation (10), $w_{1}$ (resp. $w_{2}$ ) meets the conditions in the brackets on the RHS of this equation and verifies that $v+1$ is in $C_{1}$ (resp. $C_{2}$ ). This demonstrates that $C_{1} \cap C_{2} \neq \emptyset$. On the other hand, if $T(v)=0$, pick some $u$ such that $\langle u, v\rangle \in E(\theta \omega)$ (there is at least one such $u$, since every node has at least one other node that leads to it in the computation graph). By equation (25) we 
have $v+1 \in F_{C_{1}}(\omega) \cap F_{C_{2}}(\omega)$, and since $T(v+1)=1>0$, it meets the conditions in the brackets on the RHS of equation (10) verifying that $u+1$ is both in $C_{1}$ and in $C_{2}$. In this case also, $C_{1} \cap C_{2} \neq \emptyset$. This completes the proof of (1).

To prove (2), for any $w \in \Omega_{0}$ define

$$
\mathcal{Y}(\omega)=\left\{\left(C_{a}, C_{b}\right): C_{a}, C_{b} \in \mathcal{S}(\omega), C_{a} \cap C_{b}=\emptyset\right\}
$$

Then (1), which was just proved, shows that

$$
|\mathcal{Y}(\theta \omega)| \geq|\mathcal{Y}(\omega)| \text { for all } \omega \in \Omega_{0}
$$

But now we may use ergodicity, as in the proof of Theorem 2 to conclude that $|\mathcal{Y}(\omega)|$ is almost surely constant. Together with (1), this establishes (2).

From now on, let $\Omega_{1}$ denote an a.s.s.i. subset of $\Omega_{0}$ on which (1) and (2) hold for all distinct $C_{1}, C_{2} \in \mathcal{S}(\omega)$. Note that, for $\omega \in \Omega_{1}$,

$$
C_{1} \cap C_{2}=\emptyset \Longleftrightarrow \theta^{n} C_{1} \cap \theta^{n} C_{2}=\emptyset \text { for all } n \in \mathbf{Z} \text {. }
$$

To prove (3), let $\omega \in \Omega_{1}$ and let $C_{1}, C_{2} \in \mathcal{S}(\omega)$ be such that $C_{1} \cap C_{2}=\emptyset$. Suppose that $F_{C_{1}}(\omega) \cap F_{C_{2}}(\omega) \neq \emptyset$. Let $u \in F_{C_{1}}(\omega) \cap F_{C_{2}}(\omega)$, and let $T(u)=n$. Then, by equation (23) we have $u-n \in F_{\theta^{n} C_{1}}\left(\theta^{n} \omega\right) \cap F_{\theta^{n} C_{2}}\left(\theta^{n} \omega\right)$. Since $T(u-n)=0$, by equation (24) and Lemma 1, part (2), we have $u-n \in \theta^{n} C_{1} \cap \theta^{n} C_{2}$. Hence $\theta^{n} C_{1} \cap \theta^{n} C_{2} \neq \emptyset$, which contradicts equation (26). This establishes (3).

To prove (4), let $\omega \in \Omega_{1}$ and let $C_{1} \cap C_{2} \neq \emptyset$. By equation (26), we have $\theta^{n} C_{1} \cap \theta^{n} C_{2} \neq \emptyset$ for all $n \in \mathbf{Z}$. By equation (25) this implies that $\left|F_{C_{1}}(\omega) \cap F_{C_{2}}(\omega)\right|=\infty$. However, for any $u \in F_{C_{1}}(\omega) \cap F_{C_{2}}(\omega), C(u) \subseteq C_{1} \cap C_{2}$. Since there are infinitely many such $u$, an infinite number of them must have the same color $C_{3} \subseteq C_{1} \cap C_{2}$, where $C_{3} \in \mathcal{S}(\omega)$. This establishes (4). Note that when either $C_{1}$ or $C_{2}$ is a subset of the other, $C_{3}$ may equal the smaller one of them.

To prove (5), let $\omega \in \Omega_{0}$, and let $C_{1}, C_{2} \in \mathcal{S}(\omega)$ be such that $C_{1} \nsubseteq C_{2}$, and pick $u \in C_{1} \backslash C_{2}$. We consider two cases.

In the first case, if $u-1 \in \theta C_{1}$, then we claim that $u-1 \notin \theta C_{2}$. This would establish that $\theta C_{1} \nsubseteq \theta C_{2}$. It suffices to note that since $u \in W$, if $u-1 \in \theta C_{2}$, then by Lemma 3 we have $u \in C_{2}$, a contradiction.

In the second case, if $u-1 \notin \theta C_{1}$, then we claim that there exists a node $v$ such that $T(v)=1,\left\langle u, v>\in E(\omega), v-1 \in \theta C_{1}\right.$, and $v-1 \notin \theta C_{2}$. The existence of such a node 
would establish that $\theta C_{1} \nsubseteq \theta C_{2}$. Since $u \in C_{1}$ and $u-1 \notin \theta C_{1}$, by Lemma 4 we may find $v \in F_{C_{1}}(\omega)$ such that $\langle u, v\rangle \in E(\omega), T(v)=1$, and $v-1 \in \theta C_{1}$. Suppose $v-1 \in \theta C_{2}$. By equation (25) this means $v \in F_{C_{2}}(\omega)$ and so, by equation (10) $u \in C_{2}$, a contradiction.

This completes the proof of (5).

To prove (6), for any $w \in \Omega_{1}$ define

$$
\mathcal{Z}(\omega)=\left\{\left(C_{a}, C_{b}\right): C_{a}, C_{b} \in \mathcal{S}(\omega), C_{a} \neq C_{b}, \text { and } C_{a} \nsubseteq C_{b}\right\}
$$

Then (5), which was just proved, shows that

$$
|\mathcal{Z}(\theta \omega)| \geq|\mathcal{Z}(\omega)| \text { for all } \omega \in \Omega_{0}
$$

But now we may use ergodicity, as in the proof of Theorem 2 to conclude that $|\mathcal{Z}(\omega)|$ is almost surely constant. Together with (5), this establishes (6).

From now on let $\Omega_{1}$ denote an a.s.s.i. subset of $\Omega_{0}$ on which (1) through (6) hold for all distinct $C_{1}, C_{2} \in \mathcal{S}(\omega)$. Note that, for $\omega \in \Omega_{1}$, in addition to equation (26) we also have, for each $C_{1}, C_{2} \in \mathcal{S}(\omega)$,

$$
C_{1} \subseteq C_{2} \Longleftrightarrow \theta^{n} C_{1} \subseteq \theta^{n} C_{2} \text { for all } n \in \mathbf{Z}
$$

To prove (7), let $\omega \in \Omega_{1}$ and let $C_{1}, C_{2} \in \mathcal{S}(\omega)$ be such that $C_{1} \subseteq C_{2}$. Suppose that $F_{C_{1}}(\omega) \nsubseteq F_{C_{2}}(\omega)$, and let $u \in F_{C_{1}}(\omega)$ be such that $u \notin F_{C_{2}}(\omega)$. Let $T(u)=n$. Since $T(u-n)=0$, and $u \in F_{C_{1}}(\omega)$, by equation(24) we have $u \in \theta^{n} C_{1}$, and since $u \notin F_{C_{2}}(\omega)$, by equation(25) we have $u \notin \theta^{n} C_{2}$. This contradicts equation (27). This establishes (7) and completes the proof of the proposition.

Remark 8 From now on $\Omega_{0}$ denotes an a.s.s.i. subset of scenarios for which the claims of Proposition 4 hold. As indicated in Remark 5, we may also assume, without loss of generality, that all $\omega \in \Omega_{0}$ also satisfy Proposition 2 .

\subsection{An example}

The example of this subsection will illustrate some of the properties proved in Proposition 4.

Let $A$ be a $2 \times 2$ matrix with no zero elements. Let $B=4$ and $\Omega=\left\{\omega_{0}, \omega_{1}, \omega_{2}, \omega_{3}\right\}$ with $P\left(\omega_{i}\right)=1 / 4$ and $\theta \omega_{i}=\omega_{(i+1) \bmod 4}$ for $0 \leq i \leq 3$. Clearly, $(P, \theta)$ is stationary and ergodic. 
Let $d\left(n, \omega_{i}\right)=d\left(n \bmod 4, \omega_{i}\right)$ (that is, the delays are periodic with period 4$)$ with

$$
d\left(1, \omega_{0}\right)=d\left(2, \omega_{0}\right)=\left(\begin{array}{ll}
1 & 1 \\
1 & 1
\end{array}\right), \quad d\left(3, \omega_{0}\right)=\left(\begin{array}{ll}
4 & 1 \\
1 & 4
\end{array}\right), \quad d\left(4, \omega_{0}\right)=\left(\begin{array}{ll}
4 & 4 \\
4 & 4
\end{array}\right),
$$

and $d\left(n, \omega_{i}\right)=d\left((n+1) \bmod 4, \omega_{(i-1) \bmod 4}\right)$ to make the delays compatible with $\theta$. Then, by induction on sections of the graph with temporal length of 4 , we can show that $\mathcal{S}\left(\omega_{0}\right)=$ $\left\{C_{1}, C_{2}, C_{3}\right\}$, with

$$
\begin{gathered}
C_{1}=\{(1,0),(2,0)\}, \\
C_{2}=\{(1,0),(2,0),(1,-1)\}, \\
C_{3}=\{(1,0),(2,0),(2,-1)\},
\end{gathered}
$$

and

$$
\begin{gathered}
F_{C_{1}}\left(\omega_{0}\right)=\{(1,4+4 n),(2,4+4 n),(1,1+4 n),(2,1+4 n): n \in \mathbf{Z}\}, \\
F_{C_{2}}\left(\omega_{0}\right)=\{(1,4+4 n),(2,4+4 n),(1,3+4 n),(2,2+4 n),(1,1+4 n),(2,1+4 n): n \in \mathbf{Z}\}, \\
F_{C_{3}}\left(\omega_{0}\right)=\{(1,4+4 n),(2,4+4 n),(2,3+4 n),(1,2+4 n),(1,1+4 n),(2,1+4 n): n \in \mathbf{Z}\} .
\end{gathered}
$$

In this example, we have

$$
\begin{gathered}
\theta C_{1}=\{(1,-1),(2,-1)\}, \\
\theta C_{2}=\{(1,0),(2,0),(1,-1),(2,-1),(1,-2)\}, \\
\theta C_{3}=\{(1,0),(2,0),(1,-1),(2,-1),(2,-2)\} .
\end{gathered}
$$

and, using $G\left(\omega_{1}\right)$, one can verify that $\mathcal{S}\left(\omega_{1}\right)=\left\{\theta C_{1}, \theta C_{2}, \theta C_{3}\right\}$.

We now verify some of the statements of Proposition 4 in this example. To see that Proposition 4, part (2), holds, note that $C_{1} \cap C_{2}=\{(1,0),(2,0)\}$ and $\theta C_{1} \cap \theta C_{2}=$ $\{(1,-1),(2,-1)\}$; to see that Proposition 4, part (4), holds, note that $C_{1} \subseteq C_{2} \cap C_{3}$ and $\theta C_{1} \subseteq \theta C_{2} \cap \theta C_{3}$; to see that Proposition 4, part (5) and part (6), hold note that $C_{1}$ is a subset of both $C_{2}$ and $C_{3}$ and neither $C_{2}$ nor $C_{3}$ is a subset of the other, and respectively, $\theta C_{1}$ is a subset of both $\theta C_{2}$ and $\theta C_{3}$ and neither $\theta C_{2}$ nor $\theta C_{3}$ is a subset of the other.

Remark 9 For certain examples, Proposition 4 allows us to a.s. canonically distinguish the colors of $\mathcal{S}$. For instance, in the example of subsection 2.4, where $|\mathcal{S}|=2$, we had that one color of $\mathcal{S}$ was a proper subset of the other color. This holds for each of the two scenarios $\omega_{0}$ and $\omega_{1}: C_{1} \subseteq C_{2}$ and $C_{1}^{\prime} \subseteq C_{2}^{\prime}$. Therefore, given an $\omega \in\left\{\omega_{0}, \omega_{1}\right\}$, we can 
define random function $C_{a}(\omega)$ to be the $C \in \mathcal{S}(\omega)$ such that $C \subseteq C^{\prime}$ for $C^{\prime} \in \mathcal{S}(\omega) \backslash\{C\}$, and $C_{b}(\omega)$ to be the $C \in \mathcal{S}(\omega)$ such that $C \nsubseteq C_{a}(\omega)$. Note that, in general, if we have two colors in the spectrum, one of which is a proper subset of the other on a set of scenarios of positive probability, then the results we have proved so far show that this must be the case a.s., and we can then similarly canonically distinguish between the smaller color and the larger color.

However, an analogous procedure may not always be possible. For instance, in the example of subsection 4.2 we had for $\omega_{0}$ that $C_{1} \subseteq C_{2} \cap C_{3}$, and neither $C_{2}$ nor $C_{3}$ were subsets of each other. Since $P\left(\omega_{0}\right)=1 / 4$, by parts (3), (4), and (5) of Proposition 4 and ergodicity, such relationship must be preserved among the three colors of the spectrum of all four scenarios. This allows us to canonically differentiate the smaller color from the other two for all scenarios, while the other two colors cannot be canonically differentiated based on the results proved so far.

\subsection{Lexicographic ordering}

Lexicographically order the nodes of the base as

$$
(1,0)<(2,0)<\ldots<(p, 0)<(1,-1)<\ldots<(p,-1)<\ldots<(p,-B+1) .
$$

The colors of the spectrum are just subsets of the base. We may therefore lexicographically order them. On the a.s.s.i. subset of scenarios $\Omega_{0}$ we now have well defined functions $C_{1}(\omega), \ldots, C_{\sigma}(\omega)$.

It is important to realize that $C_{i}(\theta \omega) \neq \theta C_{i}(\omega)$ in general, see Remark 9. Let $\mathcal{P}_{\sigma}$ denote the set of permutations of $\{1, \ldots, \sigma\}$. On $\Omega_{0}$ we define the $\mathcal{P}_{\sigma}$-valued random variable $\pi(\omega)$ by the rule that

$$
\pi(\omega)[i]=j \text { if and only if } \theta C_{i}(\omega)=C_{j}(\theta \omega)
$$

Equation (28) may be alternately expressed as

$$
\theta C_{i}(\omega)=C_{\pi(\omega)[i]}(\theta \omega)
$$

\subsection{The frequency of intersections : an alternative}

The following result will be very useful in the subsequent discussion : 
Lemma 7 For an a.s.s.i. subset of scenarios, the following is true for every pair of disjoint subsets of colors $\mathcal{C}_{1}$ and $\mathcal{C}_{2}$ :

$$
\begin{array}{r}
\text { Either }\left|\left\{n \in \mathbf{Z}: \bigcap_{C_{i} \in \mathcal{C}_{1}} \theta^{n} C_{i} \backslash \bigcup_{C_{j} \in \mathcal{C}_{2}} \theta^{n} C_{j} \neq \emptyset\right\}\right|=\infty \\
\text { or }\left|\left\{n \in \mathbf{Z}: \bigcap_{C_{i} \in \mathcal{C}_{1}} \theta^{n} C_{i} \backslash \bigcup_{C_{j} \in \mathcal{C}_{2}} \theta^{n} C_{j} \neq \emptyset\right\}\right|=0 .
\end{array}
$$

Proof : Let $\omega \in \Omega_{0}$. Thus $|\mathcal{S}(\omega)|=\sigma$. Lexicographically order the subset of the base, and thus the colors, as in subsection 4.3.

Let $\mathcal{H}$ denote the set of all ordered pairs of the form $(\mathcal{I}, \mathcal{J})$ where $\mathcal{I}$ and $\mathcal{J}$ are disjoint subsets of $\{1, \ldots, \sigma\}$. For $h \in \mathcal{H}$ and $\pi \in \mathcal{P}_{\sigma}$ we define $\pi[h]$ to be the pair $(\{\pi[i]: i \in \mathcal{I}\},\{\pi[j]: j \in \mathcal{J}\})$.

Next, for $\omega \in \Omega_{0}$ and $h \in \mathcal{H}$, define

$$
\mathcal{N}(h, \omega)=\max \left\{n: \bigcap_{i \in \mathcal{I}} \theta^{n} C_{i} \backslash \bigcup_{j \in \mathcal{J}} \theta^{n} C_{j} \neq \emptyset\right\}
$$

Then we have

$$
\mathcal{N}(\pi(\omega)[h], \theta \omega)=\mathcal{N}(h, \omega)-1
$$

It follows that, for each $k \in \mathbf{Z}$, we have that $|\{h \in \mathcal{H}: N(h, \omega) \leq k\}|$ is invariant, and therefore has probability 0 or 1 . From this and equation (31) it follows that for each $h \in \mathcal{H}, \mathcal{N}(h, \omega)$ almost surely only takes on the values $\infty$ or $-\infty$. The claim is proved.

Remark 10 In the statement of Lemma 7, when the first alternative in equation (30) holds, it may still not be true that $\bigcap_{C_{i} \in \mathcal{C}_{1}} \theta^{n} C_{i} \backslash \bigcup_{C_{j} \in \mathcal{C}_{2}} \theta^{n} C_{j} \neq \emptyset$ for every $n \in \mathbf{Z}$. For instance, consider the example of subsection 4.2. Let $\mathcal{C}_{1}=\left\{C_{2}, C_{3}\right\}$ and $\mathcal{C}_{2}=\left\{C_{1}\right\}$. Then $\left(C_{2} \cap C_{3}\right) \backslash C_{1}=\emptyset$ whereas $\left(\theta C_{2} \cap \theta C_{3}\right) \backslash \theta C_{1}=\{(1,0),(2,0)\}$.

Remark 11 From now on, $\Omega_{0}$ denotes an a.s.s.i. subset of scenarios on which Lemma 7 holds. Without loss of generality, we will also assume that all $\omega \in \Omega_{0}$ also satisfy all earlier propositions.

\subsection{Invariance of the inclusion structure of colors}

On the a.s.s.i. subset of scenarios $\Omega_{0}$, we know that the number of colors of the spectrum is a nonrandom constant $\sigma$. Choose an arbitrary indexing of the $\sigma$ colors of the spectrum. 
The inclusion partial order on colors induces a partial order on $\{1, \ldots, \sigma\}$. We consider the equivalence class of this order under reindexing of the colors as a random variable $\Xi(\omega)$ defined on $\Omega_{0}$. The main result of this section is the following :

Theorem 3 There is a nonrandom constant such that $\Xi(\omega)$ equals this constant for all $\omega \in \Omega_{0}$.

Proof : Assume that the colors are lexicographically ordered as described at the beginning of subsection 4.3. On the a.s.s.i. subset of scenarios $\Omega_{0}$, we now have well defined functions $C_{1}(\omega), \ldots, C_{\sigma}(\omega)$. We will write $i<_{\omega} j$ if $C_{i}(\omega) \subseteq C_{j}(\omega)$. A partial order on $\{1, \ldots, \sigma\}$, denoted $<$, belongs to the same equivalence class as the partial order $<_{\omega}$ if and only if there is some permutation $\pi \in \mathcal{P}_{\sigma}$ such that, for all $i, j \in\{1, \ldots, \sigma\}$,

$$
i<j \text { if and only if } \pi[i]<_{\omega} \pi[j] .
$$

Recall the definition of the random permutation $\pi(\omega)$, defined for $\omega \in \Omega_{0}$, in equation (28). From equation (29) and parts (5) and (6) of Proposition 4, we see that for for any $i, j \in\{1, \ldots, \sigma\}, i<_{\omega} j$ if and only if $\pi(\omega)[i]<_{\theta \omega} \pi(\omega)[j]$. Comparing with equation (32), this says that the partial order $<_{\omega}$ is in the same equivalence class as the partial order $<_{\theta \omega}$. This is precisely what is being claimed in the theorem.

\section{$5 \quad$ Pure Colors}

In Theorem 3 we have proved that, on the a.s.s.i. subset of scenarios $\Omega_{0}$, the inclusion partial order on the colors of the spectrum is shift invariant, modulo reindexing of the colors. In this section we first introduce some notation for the level sets of this partial order, to facilitate subsequent discussions. The main result of this section is the identification of a particular kind of color, called a pure color, which will play a significant role in our description of convergence rates of the partially asynchronous iteration in Part II.

Definition $11\left(\mathcal{S}_{i}(\omega)\right)$ For $\omega \in \Omega_{0}$, and $i \geq 1$, inductively define the random sets $\mathcal{S}_{i}(\omega) \subseteq \mathcal{S}(\omega)$ as follows:

$$
\mathcal{S}_{i}(\omega)=\left\{\begin{aligned}
\left\{C \in \mathcal{S}(\omega): C^{\prime} \in \mathcal{S}(\omega) \text { and } C^{\prime} \subseteq C \Longrightarrow C^{\prime}=C\right\}, & \text { if } i=1 ; \\
\left\{C \in \mathcal{S}(\omega) \backslash \bigcup_{j=1}^{i-1} \mathcal{S}_{j}(\omega):\right. & \\
\left.C^{\prime} \in \mathcal{S}(\omega) \backslash \bigcup_{j=1}^{i-1} \mathcal{S}_{j}(\omega) \text { and } C^{\prime} \subseteq C \Longrightarrow C^{\prime}=C\right\}, & \text { otherwise. }
\end{aligned}\right.
$$


Note that, since the spectrum is nonempty, $\mathcal{S}_{1}(\omega) \neq \emptyset$. Further, there is some $\rho(\omega) \leq$ $|\mathcal{S}(\omega)|$ such that $\mathcal{S}_{i}(\omega) \neq \emptyset$ for $i=1, \ldots, \rho(\omega)$ and $\left\{\mathcal{S}_{i}(\omega)\right\}_{i=1}^{\rho(\omega)}$ partitions $\mathcal{S}(\omega)$. For $i \geq 1$, let $\sigma_{i}(\omega)$ denote $\left|\mathcal{S}_{i}(\omega)\right|$.

The random variables $\rho(\omega)$ and $\sigma_{i}(\omega)$ can be thought of as functions of $\Xi(\omega)$. From Theorem 3, the following result is now immediate :

Lemma 8 Let $\omega \in \Omega_{0}$. There are nonrandom constants $\rho \geq 1$ and $\sigma_{i}, i \geq 1$, such that $\rho(\omega)$ and $\sigma_{i}(\omega), i \geq 1$, equal $\rho$ and $\sigma_{i}, i \geq 1$ respectively, for all such $\omega$. Also we have :

$$
\begin{aligned}
& \mathcal{S}_{i}(\theta \omega)=\theta \mathcal{S}_{i}(\omega) \text { for all } \omega \in \Omega_{0}, \quad i \geq 1 \\
& \sigma_{i}>0 \text { for } 1 \leq i \leq \rho, \\
& \sigma_{i}=0 \text { for } i \geq \rho+1, \text { and } \\
& \sum_{i=1}^{\rho} \sigma_{i}=\sigma .
\end{aligned}
$$

Proof : Given a partial order on $\{1, \ldots, \sigma\}$, consider the new partial order on $\{1, \ldots, \sigma\}$ that results when we apply a permutation $\pi \in \mathcal{P}_{\sigma}$ to the elements. The number of levels of the original partial order, and the cardinality of each of its levels, are the same as that for the new partial order. Further, the set of elements in level $i$ of the latter partial order is precisely

$$
\{\pi[k]: k \text { is at level } i \text { in the original partial order }\} \text {. }
$$

All the statements of the lemma are now immediate consequences of Theorem 3.

In the next formal definition, for each $C \in \mathcal{S}(\omega)$ we define two subsets of $\mathcal{S}(\omega)$ denoted $\mathcal{S}_{C}(\omega)$ and $\mathcal{S}_{C}^{+}(\omega)$ respectively. $\mathcal{S}_{C}(\omega)$ consists of those colors in $\mathcal{S}(\omega)$ that are properly contained in $C \cdot \mathcal{S}_{C}^{+}(\omega)$ consists of those colors in $\mathcal{S}(\omega)$ at levels no bigger than that of $C$, other than $C$ itself, that have a nonempty intersection with $C$.

Definition $12\left(\mathcal{S}_{C}(\omega)\right.$ and $\left.\mathcal{S}_{C}^{+}(\omega)\right)$ Let $\omega \in \Omega_{0}$ and consider a color $C \in \mathcal{S}_{i}(\omega)$. We define:

$$
\begin{aligned}
& \mathcal{S}_{C}(\omega)=\left\{C^{\prime} \in \mathcal{S}(\omega): C^{\prime} \subseteq C, C^{\prime} \neq C\right\}, \text { and } \\
& \mathcal{S}_{C}^{+}(\omega)=\left\{C^{\prime} \in \bigcup_{j=1}^{i} \mathcal{S}_{j}(\omega): C^{\prime} \cap C \neq \emptyset \text { and } C^{\prime} \neq C\right\} .
\end{aligned}
$$

Note that

$$
\mathcal{S}_{C}(\omega) \subseteq \bigcup_{j=1}^{i-1} \mathcal{S}_{j}(\omega) \quad \mathcal{S}_{C}^{+}(\omega) \subseteq \bigcup_{j=1}^{i} \mathcal{S}_{j}(\omega) \quad \text { and } \quad \mathcal{S}_{C}(\omega) \subseteq \mathcal{S}_{C}^{+}(\omega)
$$


Lemma 9 Let $\omega \in \Omega_{0}$ and consider a color $C \in \mathcal{S}(\omega)$. Then we have :

$$
\begin{aligned}
& \mathcal{S}_{\theta C}(\theta \omega)=\theta \mathcal{S}_{C}(\omega), \\
& \mathcal{S}_{\theta C}^{+}(\theta \omega)=\theta \mathcal{S}_{C}^{+}(\omega),
\end{aligned}
$$

Proof : Let $C \in \mathcal{S}_{i}(\omega)$. For $\omega \in \Omega_{0}$, we know that $\mathcal{S}(\theta \omega)=\{\theta C: C \in \mathcal{S}(\omega)\}$. Hence, we may start with the definition of $\mathcal{S}_{\theta C}(\theta \omega)$ and write

$$
\begin{aligned}
\mathcal{S}_{\theta C}(\theta \omega) & =\left\{\theta C^{\prime}: C^{\prime} \in \mathcal{S}(\omega), \theta C^{\prime} \subseteq \theta C, \theta C^{\prime} \neq \theta C\right\} \\
& =\theta\left\{C^{\prime}: C^{\prime} \in \mathcal{S}(\omega), C^{\prime} \subseteq C, \quad C^{\prime} \neq C\right\} \\
& =\theta \mathcal{S}_{C}(\omega)
\end{aligned}
$$

where the second step is by parts (5) and (6) of Proposition 4. This proves equation (36).

Similarly, since $\theta C \in \mathcal{S}_{i}(\theta \omega)$ by equation (34), we may write

$$
\begin{aligned}
\mathcal{S}_{\theta C}^{+}(\theta \omega) & =\left\{\theta C^{\prime}: C^{\prime} \in \mathcal{S}(\omega), \theta C^{\prime} \in \bigcup_{j=1}^{i} \mathcal{S}_{j}(\theta \omega), \theta C^{\prime} \cap \theta C \neq \emptyset, \theta C^{\prime} \neq \theta C\right\} \\
& =\theta\left\{C^{\prime}: C^{\prime} \in \mathcal{S}(\omega), C^{\prime} \in \bigcup_{j=1}^{i} \mathcal{S}_{j}(\omega), C^{\prime} \cap C \neq \emptyset, C^{\prime} \neq C\right\} \\
& =\theta \mathcal{S}_{C}^{+}(\omega)
\end{aligned}
$$

where the second step uses equation (34) and parts (1) and (2) of Proposition 4. This proves equation (37) and completes the proof of the lemma.

The following definition identifies a key type of color.

Definition 13 (Pure color) Let $\omega \in \Omega_{0}$ and consider a color $C \in \mathcal{S}(\omega)$. $C$ is said to be a pure color if and only if $C \backslash \bigcup_{C^{\prime} \in \mathcal{S}_{C}^{+}(\omega)} C^{\prime} \neq \emptyset$. Otherwise, we say that $C$ is not pure. $\diamond$

For scenarios $\omega \in \Omega_{0}$, if $C_{1} \cap C_{2} \neq \emptyset$ for some $C_{1}, C_{2} \in \mathcal{S}_{1}(\omega)$ with $C_{1} \neq C_{2}$, then, by part (4) of Proposition 4, there is some $C_{3} \in \mathcal{S}(\omega)$ such that $C_{3} \in C_{1} \cap C_{2}$. This contradicts the assumption that $C_{1}, C_{2} \in \mathcal{S}_{1}(\omega)$. In other words, for all $C \in \mathcal{S}_{1}$ we have $\mathcal{S}_{C}^{+}(\omega)=\emptyset$. The conclusion is that

$$
\text { Every } C \in \mathcal{S}_{1}(\omega) \text { is pure. }
$$

Thus, for $\omega \in \Omega_{0}$, there are always some colors that are pure, and the concept is not a vacuous one.

Some basic properties of the notion of purity under the shift are gathered in the following result : 
Lemma 10 For an a.s.s.i. subset of scenarios the following are true, for all $C \in \mathcal{S}(\omega)$, :

$$
C \in \mathcal{S}(\omega) \text { is pure } \Longleftrightarrow \theta C \in \mathcal{S}(\theta \omega) \text { is pure }
$$

and

$$
C \subseteq \bigcup_{C^{\prime} \in \mathcal{S}_{C}^{+}} C^{\prime} \text { implies } F_{C}(\omega) \subseteq \bigcup_{C^{\prime} \in \mathcal{S}_{C}^{+}} F_{C^{\prime}}(\omega)
$$

Proof : Let $\omega \in \Omega_{0}$, and let $C \in \mathcal{S}(\omega)$ be pure, i.e. $C \nsubseteq \bigcup_{C^{\prime} \in \mathcal{S}_{C}^{+}} C^{\prime}$. We will first show that $\theta C \nsubseteq \bigcup_{C^{\prime} \in \mathcal{S}_{C}^{+}} \theta C^{\prime}$. Let $u \in C, u \notin \bigcup_{C^{\prime} \in \mathcal{S}_{C}^{+}} C^{\prime}$. By Lemma 3 we have $u-1 \notin \bigcup_{C^{\prime} \in \mathcal{S}_{C}^{+}} \theta C^{\prime}$. If, in addition, $u-1 \in \theta C$, we are done. If $u-1 \notin \theta C$, by Lemma 4 we may find $v \in F_{C}(\omega)$ such that $\left\langle u, v>\in E(\omega), T(v)=1\right.$, and $v-1 \in \theta C$. If $v-1 \in \bigcup_{C^{\prime} \in \mathcal{S}_{C}^{+}} \theta C^{\prime}$, by equation (25) this mean $v \in \bigcup_{C^{\prime} \in \mathcal{S}_{C}^{+}} F_{C^{\prime}}(\omega)$, and so, by equation (10) $u \in \bigcup_{C^{\prime} \in \mathcal{S}_{C}^{+}} C^{\prime}$, a contradiction. Thus again, we would have $\theta C \nsubseteq \bigcup_{C^{\prime} \in \mathcal{S}_{C}^{+}} \theta C^{\prime}$.

Let

$$
\mathcal{Z}(\omega)=\left\{C \in \mathcal{S}(\omega): C \nsubseteq \bigcup_{C^{\prime} \in \mathcal{S}_{C}^{+}} C^{\prime}\right\}
$$

By equation (37) what we have just shown in the preceding paragraph is that, for $\omega \in \Omega_{0}$,

$$
|\mathcal{Z}(\theta \omega)| \geq|\mathcal{Z}(\omega)|
$$

Equality in equation (42) for an a.s. subset of scenarios follows by the method of proof of Theorem 2. This completes the proof of equation (39).

To verify equation (40), assume $C$ is not pure and that there is some node $u \in F_{C}(\omega)$, $u \notin \bigcup_{C^{\prime} \in \mathcal{S}_{C}^{+}} F_{C^{\prime}}(\omega)$. Let $T(u)=n$. Then, since $T(u-n)=0$ and $u \in F_{C}(\omega)$, by equation (24) we have $u \in \theta^{n} C$. Also, since $u \notin \bigcup_{C^{\prime} \in \mathcal{S}_{C}^{+}} F_{C^{\prime}}(\omega)$, from equation (25) we have $u \notin \bigcup_{C^{\prime} \in \mathcal{S}_{C}^{+}} \theta^{n} C^{\prime}$. Thus we get $\theta^{n} C \nsubseteq \bigcup_{C^{\prime} \in \mathcal{S}_{C}^{+}} \theta^{n} C^{\prime}$, which contradicts equation (39). Thus equation (40) must hold. This completes the proof of the lemma.

Remark 12 From now on $\Omega_{0}$ denotes an a.s.s.i. subset of scenarios on which the conclusions of Lemma 10 hold. As usual, without essential loss of generality, all preceding propositions will be assumed to hold for all the scenarios in $\Omega_{0}$.

In Definition 13 a pure color of level $i$ is defined as one that is not contained in the union of the colors of level $i$ or less other than itself. Rather remarkably, it turns out that an equivalent characterization is that a color is pure if it is not the union of the colors of level $i-1$ or less that it properly contains. This is the main result of this section. 
Theorem 4 For $\omega \in \Omega_{0}$ we have

$$
C \subseteq \bigcup_{C^{\prime} \in \mathcal{S}_{C}^{+}} C^{\prime} \Longleftrightarrow C=\bigcup_{C^{\prime} \in \mathcal{S}_{C}} C^{\prime}
$$

Proof : Let $C \in \mathcal{S}_{i}(\omega)$ and assume that $C \subseteq \cup_{C^{\prime} \in \mathcal{S}_{C}^{+}} C^{\prime}$ and $u \in C \backslash \cup_{C^{\prime} \in \mathcal{S}_{C}} C^{\prime}$ for some $u$. By an argument like the one used in the proof of part (1) of Lemma 1, every nonnegative $B$-block must contain a node in $F_{C}$ which has $u$ in its history. All these nodes have colors which are subsets of $C$. and all but finitely many of these nodes have colors in $\mathcal{S}(\omega)$. Since we assumed that $u \notin \cup_{C^{\prime} \in \mathcal{S}_{C}} C^{\prime}$, all but finitely many of such nodes must have color $C$.

However, by equation (40), the existence of a $C$-colored $F_{C}$ node proved in the previous paragraph implies the existence of a $C$-colored $F_{C^{\prime}}$ node for some $C^{\prime} \in \mathcal{S}_{C}^{+}(\omega)$, which in turn implies $C \subseteq C^{\prime}$. But since $\mathcal{S}_{C}^{+}(\omega) \subseteq \bigcup_{j=1}^{i} \mathcal{S}_{j}(\omega) \backslash\{C\}$, we get a contradiction. This completes the proof of equation (43) and completes the proof of the theorem.

The following immediate corollary of Theorem 4 will be useful later :

Proposition 5 For $\omega \in \Omega_{0}$, if $C \in \mathcal{S}(\omega)$ is not pure, we have $F_{C}(\omega)=\bigcup_{C^{\prime} \in \mathcal{S}_{C}} F_{C^{\prime}}(\omega)$.

Proof : Equation (43) shows that $C=\bigcup_{C^{\prime} \in \mathcal{S}_{C}} C^{\prime}$. In conjunction with equation (39) and equation (34), we can conclude from equation (43) that $\theta^{n} C=\bigcup_{C^{\prime} \in \mathcal{S}_{C}} \theta^{n} C^{\prime}$ for all $n \in \mathbf{Z}$. Suppose there is some node $u \in F_{C}(\omega)$ such that $u \notin \bigcup_{C^{\prime} \in \mathcal{S}_{C}} F_{C^{\prime}}$, and suppose $T(u)=n$. Then $u-n \in \theta^{n} C$, and $u-n \notin \bigcup_{C^{\prime} \in \mathcal{S}_{C}} \theta^{n} C^{\prime}$. This contradiction completes the proof.

Given an indexing of the $\sigma$ colors of the spectrum and the partial order on $\{1, \ldots, \sigma\}$ induced by the inclusion partial order on colors, let us mark those colors that are pure. Consider the equivalence class of such marked partial orders under reindexing of colors as a random variable $\Xi^{(m)}(\omega)$, where the superscript $(m)$ is to remind us that there is a distinction between marked and unmarked indices. Note that $\Xi(\omega)$ can be thought of as a function of $\Xi^{m}(\omega)$, by ignoring the marks. We then have the following invariance result. Note that the proof of this result does not depend on Theorem 4.

Proposition 6 There is a nonrandom constant such that $\Xi^{(m)}(\omega)$ equals this nonrandom constant for $\omega \in \Omega_{0}$. 
Proof: As in the proof of Theorem 3 assume that the colors are lexicographically ordered, so that we have well defined functions $C_{1}(\omega), \ldots, C_{\sigma}(\omega)$ for $\omega \in \Omega_{0}$. A marked partial order on $\{1, \ldots, \sigma\}$ consists of a partial order on $\{1, \ldots, \sigma\}$, denoted $<$ and a subset $\mathcal{M} \subseteq\{1, \ldots, \sigma\} . \Xi^{(m)}(\omega)$ is given by the partial order $<_{\omega}$ as in the proof of Theorem 3 , and the subset $\mathcal{M}_{\omega}$ defined by saying that $i \in \mathcal{M}_{\omega}$ if and only if $C_{i}(\omega)$ is pure. A marked partial order $(<, \mathcal{M})$ belongs to the same equivalence class as $\left(<_{\omega}, \mathcal{M}_{\omega}\right)$ if and only if there is a permutation $\pi \in \mathcal{P}_{\sigma}$ such that, in addition to equation (32) we have

$$
\text { For all } i \in\{1, \ldots, \sigma\}, i \in \mathcal{M} \text { if and only if } \pi[i] \in \mathcal{M}_{\omega} \text {. }
$$

Recall the definition of the random permutation $\pi(\omega)$, defined for $\omega \in \Omega_{0}$ in equation (28). As in the proof of Theorem 3 we see that for any $i, j \in\{1, \ldots, \sigma\}$ we have $i<_{\omega} j$ if and only if $\pi(\omega)[i]<_{\theta \omega} \pi(\omega)[j]$. Also, equation (39) shows that $i \in \mathcal{M}_{\omega}$ if and only if $\pi(\omega)[i] \in \mathcal{M}_{\theta \omega}$. Hence $\left(<_{\theta \omega}, \mathcal{M}_{\theta \omega}\right)$ is in the same equivalence class of marked partial orders as $\left(<_{\omega}, \mathcal{M}_{\omega}\right)$. This is precisely what is being claimed in the proposition.

In Section 8, we shall see that only the pure colors can introduce new convergence rates in an asynchronous computation. 


\section{Finiteness of mean time to refer to the filament}

In this section, we prove a result which will reduce the study in Section 8 of the convergence of processor time values for sequences of nodes of a given color to the convergence of blocks of values on the filaments of that color. Central to this reduction is the construction of a two-sided stationary point process, defined in terms of the concepts introduced so far. In order to introduce this stationary process, we find it convenient to define a notion of extended color for the nodes in the computation graph. Unlike the color, which is defined only for nodes with positive time indices, the extended color is defined for all nodes. Further, there is an a.s. finite random time such that the extended color and the color are the same for all nodes with time indices greater than or equal to this time.

\subsection{Extended Color}

Definition 14 (Extended color) Let $\omega \in \Omega_{0}$. For each node $u \in U$, define the extended color, $\hat{C}(u, \omega)$, as follows: say that $\hat{C}(u, \omega)=C \in \mathcal{S}(\omega)$ if and only if $C\left(u-n, \theta^{n} \omega\right)=$ $\theta^{n} C \in \mathcal{S}\left(\theta^{n} \omega\right)$ for some $n \in \mathbf{Z}$ such that $T(u)>n$.

There are two potential problems with this definition. The first problem is to show that for each $u \in U$ there is some $n$ such that $C\left(u-n, \theta^{n} \omega\right) \in \mathcal{S}\left(\theta^{n} \omega\right)$. The second problem is to show that one has not simultaneously assigned two different colors to some $u \in U$. This second problem is taken care of by Lemma 11 and the first problem by Lemma 12 . Before proving these lemmas, we find it convenient to introduce the following definition.

Definition 15 For $\omega \in \Omega_{0}$, let

$$
N(\omega)=\min \{m \geq 1: C(u, \omega) \in \mathcal{S}(\omega) \text { for all } u \text { with } T(u) \geq m\}
$$

Remark 13 Recall that on $\Omega_{0}$ only finitely many nodes with positive time indices can have a color different from one in the spectrum. Thus, for $\omega \in \Omega_{0}$,

$$
1 \leq N(\omega)<\infty
$$


We now prove that Definition 14 does not end up assigning to a node two different sets as its extended color.

Lemma 11 Let $\omega \in \Omega_{0}$ and $u \in U$. Suppose $n<T(u)$ is such that $C\left(u-n, \theta^{n} \omega\right)=\theta^{n} C$, where $C \in \mathcal{S}(\omega)$. Then $C\left(u-m, \theta^{m} \omega\right)=\theta^{m} C$ for all $m \leq n$.

Proof : Assume that $C\left(u-n, \theta^{n} \omega\right)=\theta^{n} C$. It suffices to show $C\left(u-(n-1), \theta^{n-1} \omega\right)=$ $\theta^{n-1} C$, as the lemma then follows by induction. But, by equation (18), we have

$$
\begin{aligned}
C\left(u-n+1, \theta^{n-1} \omega\right) & =C\left((u-n)+1, \theta^{-1}\left(\theta^{n} \omega\right)\right) \\
& =\theta^{-1} \theta^{n} C=\theta^{n-1} C,
\end{aligned}
$$

as desired.

Thus it remains to ask if all nodes in $U$ have an extended color: The next result answers this question positively, but only in an a.s.s.i. subset of $\Omega_{0}$.

Lemma 12 For an a.s.s.i. subset of scenarios, $\hat{C}(u, \omega)$ is well defined for all $u \in U$.

Proof : Let $\omega \in \Omega_{0}$. Denote $\sup \{T(u): \hat{C}(u, \omega)$ does not exist $\}$ by $\mu(\omega)$. If $T(u)>0$ and $C(u, \omega)=C \in \mathcal{S}(\omega)$ then $\hat{C}(u, \omega)=C$, by taking $n=0$ in Definition 14. Thus, with $N(\omega)$ as in Definition $45, \hat{C}(u, \omega)$ is defined whenever $T(u) \geq N(\omega)$. In view of equation (46), this means that $P(\mu<\infty)=1$.

Now by definition, $\hat{C}(u, \omega)$ exists and equals $C \in \mathcal{S}(\omega)$ if and only if $C\left(u-n, \theta^{n} \omega\right)=$ $\theta^{n} C$ for some $n$. But this is same as $C\left((u-1)-(n-1), \theta^{n-1} \theta \omega\right)=\theta^{n-1} \theta C$. So for all $u$, for all $\omega \in \Omega_{0}$,

$$
\begin{aligned}
& \hat{C}(u, \omega) \text { exists and equals } C \in \mathcal{S}(\omega) \Longleftrightarrow \\
& \qquad \hat{C}(u-1, \theta \omega) \text { exists and equals } \theta C \in \mathcal{S}(\theta \omega) .
\end{aligned}
$$

This implies that $\mu(\theta \omega)=\mu(\omega)-1$, which in turn implies that, on $\Omega_{0}$,

$$
\theta\{\mu<n\}=\{\mu<n-1\}
$$

We therefore have

$$
\begin{aligned}
P(\mu<\infty) & =\lim _{n \rightarrow \infty} P(\mu<n) \\
& =P(\mu<n) \text { for all } n
\end{aligned}
$$


where the second equality is due to equation (48) and the stationarity of $(P, \theta)$. Therefore, since $P(\mu<\infty)=1$, we get $P(\mu<n)=1$ for all $n \in \mathbf{Z}$. Hence $\mu(\omega) \equiv-\infty$ a.s., which proves the claim.

Remark 14 From now on, $\Omega_{0}$ denotes an a.s.s.i. subset of scenarios on which Lemma 12 holds. Without loss of generality, all preceding propositions will be assumed to hold for all scenarios in $\Omega_{0}$.

\subsection{An interpretation of extended color}

Consider a node $u \in U$, and let $n<T(u) . C\left(u-n, \theta^{n} \omega\right)+n$ is seen to be the set of nodes in the $B$-block $W+n$ whose values determine the value of node $u$ at scenario $\omega$. This sentence should be interpreted in terms of a computation that starts with initial conditions for the nodes in $W+n$; note that $n$ may be strictly positive or strictly negative. The way to think about the extended color is that the largest value of $n$ for which $C\left(u-n, \theta^{n} \omega\right)$ equals $\theta^{n} C$ for some $C \in \mathcal{S}(\omega)$ corresponds to the most recent $n$-block preceding $u$ such that the value of $u$ in terms of initial conditions in this $n$-block may be calculated in an "invariant way". Lemma 11 can now be thought of as saying that if initial conditions in the $B$-block $W+n$ lead to the value of $u$ being computed in an invariant way, then this will be true also for initial conditions in any $B$-block $W+m$, where $m \leq n$.

We next make a crucial definition, which is going to be used to "put all computations on an invariant footing".

Definition 16 For $\omega \in \Omega_{0}$, define

$$
\beta(n, \omega)=\sup \left\{m: \forall u \text { with } T(u) \geq n, C\left(u-m, \theta^{m} \omega\right)=\theta^{m} \hat{C}(u, \omega)\right\}
$$

Remark 15 Since $C\left(u-m, \theta^{m} \omega\right)$ is defined only if $m<T(u)$, we have

$$
\beta(n, \omega)<n, \quad n \in \mathbf{Z} .
$$

In the language of the first paragraph of this subsection, the $\beta(n, \omega)$-block $W+\beta(n, \omega)$ is the most recent $B$-block of initial conditions relative to which the values of every one of the nodes $u$ with $T(u) \geq n$ can be computed in an invariant way. 
There is a clearly a potential problem with Definition 16 : the set in brackets on the RHS of equation (49) may be empty, which would mean that $\beta(n, \omega)=-\infty$, making the concept vacuous. The next lemma shows that this cannot be the case, in an a.s.s.i. subset of $\Omega_{0}$

Lemma 13 For an a.s.s.i. subset of scenarios, we have

(1) $\beta(n, \omega)=\beta(n-1, \theta \omega)+1$, and

(2) $\beta(n, \omega)>-\infty$ for all $n \in \mathbf{Z}$.

Proof : Let $\omega \in \Omega_{0}$. To prove (1),

$$
\begin{aligned}
& \beta(n-1, \theta \omega)=\sup \left\{m: \forall u \text { with } T(u) \geq n-1, C\left(u-m, \theta^{m}(\theta \omega)=\theta^{m} \hat{C}(u, \theta \omega)\right\}\right. \\
& \quad=\sup \left\{m: \forall u \text { with } T(u+1) \geq n, C\left((u+1)-(m+1), \theta^{m+1} \omega\right)=\theta^{m+1} \hat{C}(u+1, \omega)\right\} \\
& \quad=\beta(n, \omega)-1,
\end{aligned}
$$

where equation (47) was used in the second equality.

To prove $(2)$, define $\mu(\omega)=\sup \{n: \beta(n, \omega)=-\infty\}$. With $N(\omega)$ as in Definition 45 we have $\beta(n, \omega) \geq 0$ for all $n \geq N(\omega)$. Hence $P(\mu<\infty)=1$. On the other hand by using part (1) of the lemma, we get $\theta\{\mu<n\} \subseteq\{\mu<n-1\}$ on $\Omega_{0}$. Hence $P(\mu<n-1)=P(\mu<n)$. But $\lim _{n \rightarrow \infty} P(\mu<n)=P(\mu<\infty)=1$. Hence $P(\mu<n)=1$ for all $n \in \mathbf{Z}$. Hence $\mu(\omega) \equiv-\infty$ a.s. on $\Omega_{0}$, and part (2) of the lemma is proved.

Remark 16 From now on, $\Omega_{0}$ denotes an a.s.s.i. subset of scenarios for which Lemma 13 holds. Without loss of generality, we will also assume that all of the other results proved so far hold for every scenario in $\Omega_{0}$.

\subsection{A stationary marked point process}

Definition 17 For $\omega \in \Omega_{0}$, inductively define (with $\tau_{0}$ first)

$$
\tau_{i}(\omega)= \begin{cases}\sup \{n \leq 0: \beta(n, \omega)<\beta(n+1, \omega)\} & \text { for } i=0, \\ \sup \left\{n<\tau_{i+1}(\omega): \beta(n, \omega)<\beta(n+1, \omega)\right\} & \text { for } i<0 \\ \inf \left\{n>\tau_{i-1}(\omega): \beta(n, \omega)<\beta(n+1, \omega)\right\} & \text { for } i>0\end{cases}
$$

Remark 17 Recall the interpretation of $\beta(n, \omega)$ given in Remark 15. This yields an interpretation for the $\left(\tau_{i}(\omega), i \in \mathbf{Z}\right)$. As $n$ increases, there will be certain times $n$ at which 
the "most recent block of initial conditions that can be referred to in order to yield the values of all nodes with time index at least $n$ in an invariant way" jumps. The times $\left(\tau_{i}(\omega), i \in \mathbf{Z}\right)$ are precisely the times prior to these jumps.

Lemma 14 For all $\omega \in \Omega_{0}$, for all $i \in \mathbf{Z}, \tau_{i}(\omega)(\omega)$ is finite.

Proof : Let $\omega \in \Omega_{0}$. We first prove that $\tau_{0}(\omega)$ is finite. Since $0 \geq \tau_{0}(\omega)$ by definition, it suffices to show that $\tau_{0}(\omega)>-\infty$. According to part (2) of Lemma $13, \beta(1, \omega)>-\infty$, and so, again by part (2) of Lemma $13, \beta(\beta(1, \omega), \omega)>-\infty$. On the other hand, by equation $(50), \beta(\beta(1, \omega), \omega)<\beta(1, \omega)$ and $\beta(1, \omega)<1$. Therefore, by the definition of $\tau_{0}(\omega)$, we get $-\infty<\beta(1, \omega) \leq \tau_{0}(\omega) \leq 0$. This establishes that $\tau_{0}(\omega)$ is finite.

We next prove that for all $0>i, \tau_{i}(\omega)(\omega)$ is finite. Since, for $0 \geq i$, we have $0 \geq$ $\tau_{i}(\omega)(\omega)$ by definition, it suffices to show that $\tau_{i}(\omega)(\omega)>-\infty$ for all $0 \geq i$. We prove this by induction, starting with the result for $i=0$, which was shown in the previous paragraph. With the inductive hypothesis in place, according to part (2) of Lemma 13 and equation (50), we have $-\infty<\beta\left(\beta\left(\tau_{i}(\omega), \omega\right), \omega\right)<\beta\left(\tau_{i}(\omega), \omega\right)$ and $\beta\left(\tau_{i}(\omega), \omega\right)<$ $\tau_{i}(\omega)$. Therefore, by definition of $\tau_{i-1}(\omega)$, we get $-\infty<\beta\left(\tau_{i}(\omega), \omega\right) \leq \tau_{i-1}(\omega)<\tau_{i}(\omega)$, establishing the truth of the inductive hypothesis for $i-1$ (recall $i \leq 0$ ). This completes the proof that $\tau_{i}(\omega)(\omega)$ is finite for all $0>i$.

Finally we show that $\tau_{i}(\omega)(\omega)$ is finite for all $i>0$. We prove this by induction on $i \geq 0$, starting with the result for $i=0$, which was shown in the first paragraph. With the inductive hypothesis in place, let $\omega^{\prime}=\theta^{\tau_{i}(\omega)+1} \omega$, which is well-defined, since we have $-\infty<\tau_{i}(\omega)$ by inductive hypothesis. With $N\left(\omega^{\prime}\right)$ as in equation (45) we have $\beta\left(N\left(\omega^{\prime}\right), \omega^{\prime}\right) \geq 0$. By repeated applications of part (1) of Lemma 13, this implies $\beta\left(N\left(\omega^{\prime}\right)+\tau_{i}(\omega)+1, \omega\right) \geq \tau_{i}(\omega)+1$. But by $(50), \beta\left(\tau_{i}(\omega)+1, \omega\right)<\tau_{i}(\omega)+1$, and so we get $\beta\left(\tau_{i}(\omega)+1, \omega\right)<\beta\left(N\left(\omega^{\prime}\right)+\tau_{i}(\omega)+1, \omega\right)$. Therefore, we get $\tau_{i}(\omega)+1 \leq \tau_{i+1}(\omega)<$ $N\left(\omega^{\prime}\right)+\tau_{i}(\omega)+1<\infty$. We of course have $\tau_{i+1}(\omega) \geq \tau_{i}(\omega)>-\infty$ by inductive hypothesis. Thus the inductive hypothesis is shown to propagate to $i+1$. This completes the proof that $\tau_{i}(\omega)(\omega)$ is finite for all $i>0$ and completes the proof of the lemma.

An immediate consequence of Lemma 14 is that for $\omega \in \Omega_{0}$,

$$
\lim _{n \rightarrow \infty} \beta(n, \omega)=\infty .
$$

Now by part (1) of Lemma 13, for all $\omega \in \Omega_{0}, \beta(n, \omega)<\beta(n+1, \omega)$ if and only if $\beta(n-1, \theta \omega)<\beta(n, \theta \omega)$. This in conjunction with the stationarity of $(P, \theta)$ implies that: 


$$
\left\{\tau_{i}, i \in \mathbf{Z}\right\} \text { is a stationary point process on } \Omega_{0} \text {. }
$$

A number of random variables associated to this stationary point process are also of interest, and we proceed to define them. These can be thought of as marks associated to the points of this process.

Definition 18 Define the random variables $\nu(n), n \in \mathbf{Z}$, by

$$
\nu(n)=\max \left\{i: \tau_{i}<n\right\}+1, \quad n \in \mathbf{Z},
$$

and define the sequences of random variables $\left\{R_{i}, i \in \mathbf{Z}\right\}$ and $\left\{Z_{i}, i \in \mathbf{Z}\right\}$ by:

$$
\begin{aligned}
& R_{i}=\beta\left(\tau_{i}\right), \\
& Z_{i}=\tau_{i}-R_{i} .
\end{aligned}
$$

See Figure 1 for a picture of these constructions.

Remark $18(\nu(n), n \in \mathbf{Z})$ is just a counting process associated to the stationary point process $\left(\tau_{i}, i \in \mathbf{Z}\right)$, introduced for notational convenience in a couple of subsequent equations. As Figure 1 shows, this definition is somewhat unorthodox, but we prefer to do it this way. $R_{i}$ can be described by saying that the $B$-block $W+R_{i}$ is the most recent $B$-block of initial conditions such that all nodes with time indices at least $\tau_{i}$ can have their values computed in an invariant way relative to initial conditions in this $B$-block. The variables $Z_{i}$ are particularly interesting. They give the "depth to which it is necessary to look back to make an invariant computation for all nodes with time indices at least $\tau_{i}$ ". The main result of this section, which will be proved in the next subsection, is that the Palm stationary expectation of this random variable is finite. This is a key result, used in Section 8 to reduce the problem of the convergence rate of individual processor-time values to the convergence rates of blocks of values from filaments.

We now make a number of additional observations, which are largely straightforward. Firstly, $\nu(\cdot)$ is defined so that $\tau_{\nu(n)-1}<n \leq \tau_{\nu(n)}$. By Lemma 14, this implies that

$$
\lim _{n \rightarrow \infty} \nu(n)=\infty
$$



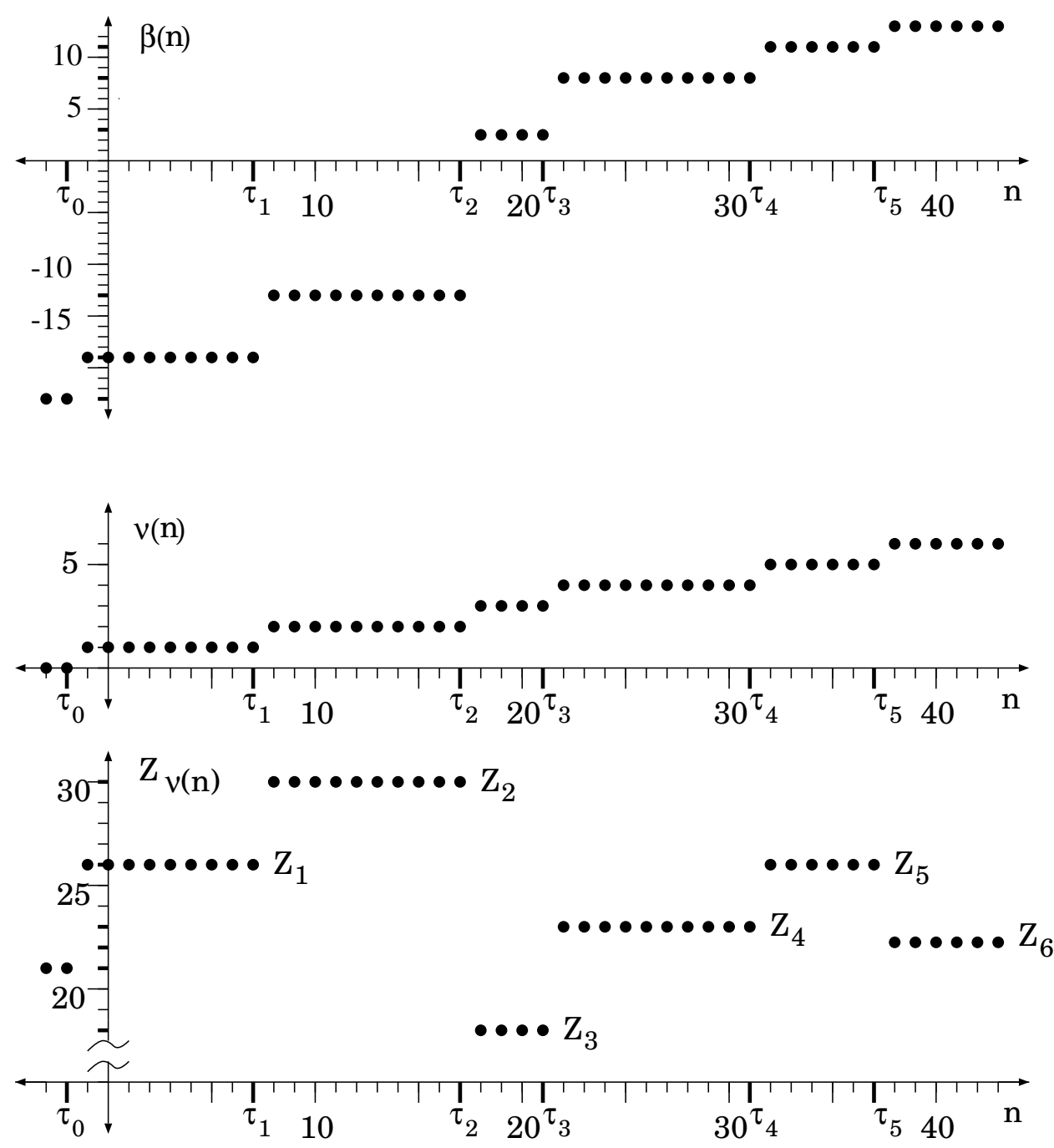

Figure 1: Constructing the processes in Section 6.3. 
Secondly, by definition of $\tau_{i}$, for $\tau_{i-1}<n_{j} \leq \tau_{i}, j=1,2$, we get $\beta\left(n_{1}\right)=\beta\left(n_{2}\right)$. This implies that $\beta(n)=\beta\left(\tau_{\nu(n)}\right)$. Also, we just saw that $n \leq \tau_{\nu(n)}$. Combining the two, we get

$$
n-\beta(n) \leq \tau_{\nu(n)}-\beta\left(\tau_{\nu(n)}\right)=Z_{\nu(n)}
$$

Thirdly, for all $i$,

$$
\tau_{i}<\tau_{i+1} \quad \text { and } \quad R_{i}<R_{i+1}
$$

The first expression is immediate. To prove the second expression, note that

$$
R_{i}=\beta\left(\tau_{i}\right)<\beta\left(\tau_{i}+1\right)=\beta\left(\tau_{i+1}\right)=R_{i+1},
$$

where the inequality is by the definition of $\tau_{i}$.

\section{4 $E_{0}\left(Z_{0}\right)$ is finite}

Our goal in this subsection is to prove Theorem 5, which refers to the Palm probability associated to the stationary sequence $\left\{\tau_{i}: i \in \mathbf{Z}\right\}$. For an introduction to Palm probabilities, see [1]. To that end, we start with proving some intermediate results.

Lemma 15 For all $i \in \mathbf{Z}$, there exists $u_{i} \in U$ with $T\left(u_{i}\right)=\tau_{i}$ such that the largest $n$ that satisfies $C\left(u_{i}-n, \theta^{n} \omega\right)=\theta^{n} \hat{C}\left(u_{i}, \omega\right)$ is $\beta\left(\tau_{i}\right)=R_{i}$.

Proof : Assume otherwise, i.e. assume that for some $i \in \mathbf{Z}$ for all $u$ with $T(u)=\tau_{i}$, there is an $n_{u}>\beta\left(\tau_{i}\right)$ such that $C\left(u-n_{u}, \theta^{n_{u}} \omega\right)=\theta^{n_{u}} \hat{C}(u, \omega)$. Then by Lemma 11 we have that, for all such $u, C\left(u-m, \theta^{m} \omega\right)=\theta^{m} \hat{C}(u, \omega)$ for all $m \leq \beta\left(\tau_{i}\right)+1$. Also, for all $u$ such that $T(u) \geq \tau_{i}+1$, we have $C\left(u-m, \theta^{m} \omega\right)=\theta^{m} \hat{C}(u, \omega)$ for all $m \leq \beta\left(\tau_{i}+1\right)$, by the definition in equation (49). But, by definition of $\tau_{i}$, we have $\beta\left(\tau_{i}\right)+1 \leq \beta\left(\tau_{i}+1\right)$. Thus we have concluded that for all $u$ such that $T(u) \geq \tau_{i}$ we have $C\left(u-m, \theta^{m} \omega\right)=\theta^{m} \hat{C}(u, \omega)$ for all $m \leq \beta\left(\tau_{i}\right)+1$. But this contradicts the definition of $\beta\left(\tau_{i}\right)$ in equation (49).

Definition 19 Define $u_{i}^{*}$ to be the $u_{i}$ having the largest processor number among those whose existence is guaranteed by Lemma 15. Note that $u_{i}^{*}$ is a random function.

Lemma 16 On $\Omega_{0}$, for all $n \in \mathbf{Z}$,

$$
\left|\left\{i: R_{i} \leq n<\tau_{i}\right\}\right| \leq 2^{B p}
$$


Proof : Fix some $\omega \in \Omega_{0}$. We first claim that for $\max \left(R_{i}, R_{j}\right) \leq n<\min \left(\tau_{i}, \tau_{j}\right)$,

$$
i \neq j \quad \Longrightarrow \quad C\left(u_{i}^{*}-n, \theta^{n} \omega\right) \neq C\left(u_{j}^{*}-n, \theta^{n} \omega\right)
$$

We prove this by contradiction. Suppose $C\left(u_{i}^{*}-n, \theta^{n} \omega\right)=C\left(u_{j}^{*}-n, \theta^{n} \omega\right)$. It follows immediately from Lemma 11 that

$$
C\left(u_{i}^{*}-m, \theta^{m} \omega\right)=C\left(u_{j}^{*}-m, \theta^{m} \omega\right), \quad m \leq n
$$

Assume with no loss of generality that $i<j$ so that $R_{i}<R_{j} \leq n<\tau_{i}<\tau_{j}$, see equation (55). By definition of $u_{j}^{*}$, we have $C\left(u_{j}^{*}-R_{j}, \theta^{R_{j}} \omega\right)=\theta^{R_{j}} \hat{C}\left(u_{j}^{*}, \omega\right)$, which by Lemma 11 implies $C\left(u_{j}^{*}-R_{i}, \theta^{R_{i}} \omega\right)=\theta^{R_{i}} \hat{C}\left(u_{j}^{*}, \omega\right)$, as $R_{i}<R_{j}$. But since $R_{i}<n$, equation (57) implies that $C\left(u_{i}^{*}-R_{i}, \theta^{R_{i}} \omega\right)=C\left(u_{j}^{*}-R_{i}, \theta^{R_{i}} \omega\right)=\theta^{R_{i}} \hat{C}\left(u_{j}^{*}, \omega\right)$. Therefore, by definition of extended color, $\hat{C}\left(u_{i}^{*}, \omega\right)=\hat{C}\left(u_{j}^{*}, \omega\right)$. Using equation (57) again (since $\left.R_{j} \leq n\right)$, we get

$$
C\left(u_{i}^{*}-R_{j}, \theta^{R_{j}} \omega\right)=C\left(u_{j}^{*}-R_{j}, \theta^{R_{j}} \omega\right)=\theta^{R_{j}} \hat{C}\left(u_{j}^{*}, \omega\right)=\theta^{R_{j}} \hat{C}\left(u_{i}^{*}, \omega\right),
$$

where the last equality is due to $\hat{C}\left(u_{i}^{*}, \omega\right)=\hat{C}\left(u_{j}^{*}, \omega\right)$. Thus we have found $R_{j}>R_{i}$ such that $C\left(u_{i}^{*}-R_{j}, \theta^{n} \omega\right)=\theta^{R_{j}} \hat{C}\left(u_{i}^{*}, \omega\right)$, which contradicts the definition of $u_{i}^{*}$. This establishes equation (56).

To prove the lemma, assume that, for some $n \in \mathbf{Z}$, there are at least $2^{B p}+1$ distinct $i$ 's such that $R_{i} \leq n<\tau_{i}$, and denote their collection by $I$. Since the maximum number of distinct subsets of the base is $2^{B p}$, for some $k \neq l, k, l \in I$, we get $C\left(u_{k}^{*}-n, \theta^{n} \omega\right)=$ $C\left(u_{l}^{*}-n, \theta^{n} \omega\right)$, which contradicts equation (56).

See Figure 2 for a graphical interpretation of Lemma 16. As a result of this lemma, we get

$$
R_{i+2 B p}>\tau_{i}, \quad i \in \mathbf{Z} .
$$

Indeed, let $R_{i+2^{B p}+1} \leq \tau_{i}$. Then for some $n, R_{i+2^{B p}+1} \leq n<\tau_{i+1}$. By equation (55), we then have

$$
R_{i+1}<R_{i+2}<\cdots<R_{i+2^{B p}+1} \leq n<\tau_{i+1}<\tau_{i+2}<\cdots<\tau_{i+2}^{B p}+1
$$

which contradicts Lemma 16.

Definition 20 Since $\left\{\tau_{i}, i \in \mathbf{Z}\right\}$ is a stationary point process, we can define its intensity $\psi$ by

$$
\psi=P\left(\tau_{i}=n \text { for some } i\right)=P(\beta(n)<\beta(n+1))
$$




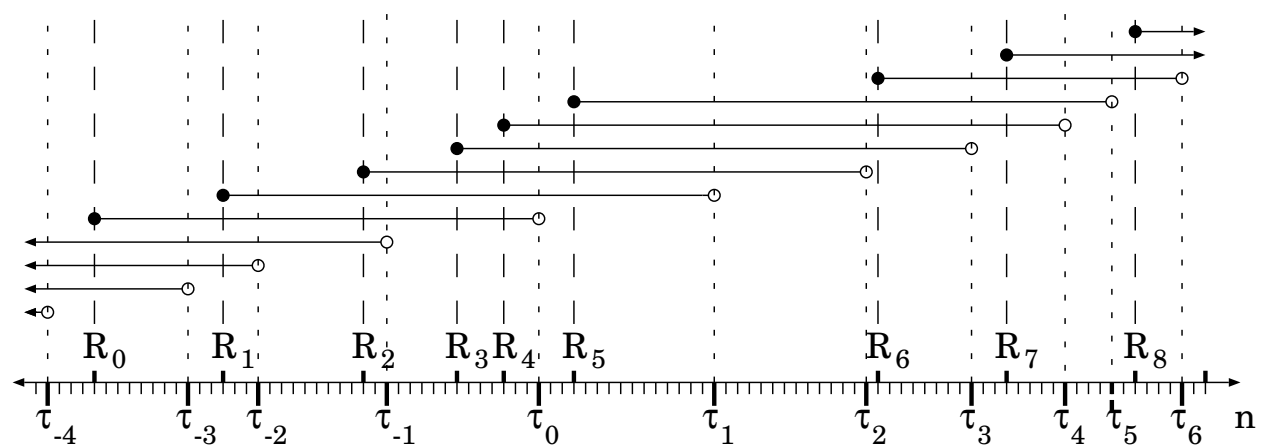

Figure 2: A graphical interpretation of Lemma 16: No matter where one draws a vertical line, it can not intersect more than $2^{B p}$ of the horizontal segments.

which, by stationarity, is constant for all $n \in \mathbf{Z}$.

The next lemma asserts that the intensity is strictly positive.

Lemma $17 \psi>0$.

Proof : Assume $\psi=0$, that is, $P(\beta(n)<\beta(n+1))=0$ for all $n$. This implies that $\sum_{n=1}^{\infty} P(\beta(n)<\beta(n+1))=0$, which, by the Borel-Cantelli Lemma, in turn implies $P(\beta(n)<\beta(n+1)$ i.o. $)=0$, contradicting Lemma 16 .

Consider now the Palm probability induced by the stationary point process $\left\{\tau_{i}, i \in \mathbf{Z}\right\}$. Let $P_{0}$ denote the Palm probability, $\theta_{0}$ denote $\theta^{\tau_{1}}$ (that is, $\theta_{0} \omega=\theta^{\tau_{1}(\omega)} \omega$ ), and $E_{0}$ denote the expectation with respect to $P_{0}$. These are defined on the set of paths where $\tau_{0} \equiv 0$. By well-known facts in Palm theory, (see (4.2.1) of [1]) we immediately conclude that

$$
E_{0}\left(\tau_{1}\right)=\psi^{-1}<\infty
$$

as $\psi>0$ by Lemma 17 . We have that $\left(P_{0}, \theta_{0}\right)$ is stationary and ergodic because $(P, \theta)$ is, by (7.2.1) of [1], and also, since $P\left(\Omega_{0}\right)=1$, by (7.1.1) of [1], we get

$$
P_{0}\left(\Omega_{0}\right)=1
$$

From equation (58) this means that, for all $i \in \mathbf{Z}$,

$$
R_{i+2 B p+1}>\tau_{i}, \quad P_{0} \text {-a.s. }
$$

We are now in a position to show the following central result: 
Theorem $5 E_{0}\left(Z_{0}\right)<\infty$.

Proof : Since $\left(P_{0}, \theta_{0}\right)$ is ergodic, we have $P_{0}$-a.s.

$$
\begin{aligned}
E_{0}\left(Z_{0}\right) & =\lim _{n \rightarrow \infty} n^{-1} \sum_{k=1}^{n} Z_{k} \\
& =\lim _{n \rightarrow \infty} n^{-1}\left[\sum_{k=1}^{2^{B p}} Z_{k}+\sum_{k=2^{B p}+1}^{n} Z_{k}\right] \\
& =\lim _{n \rightarrow \infty} n^{-1}\left[\sum_{k=2^{B p}+1}^{n} Z_{k}\right], \quad \text { since each } Z_{k}<\infty P_{0} \text {-a.s. }
\end{aligned}
$$

But $P_{0}$-a.s.

$$
\begin{aligned}
& \sum_{k=2^{B p}+1}^{n} Z_{k}=\sum_{k=2^{B p}+1}^{n}\left(\tau_{k}-R_{k}\right) \\
& =\sum_{k=2^{B p}+1}^{n} \sum_{j=-\infty}^{\infty} 1\left\{R_{k} \leq j<\tau_{k}\right\} \\
& =\sum_{j=-\infty}^{\infty} \sum_{k=2^{B p}+1}^{n} 1\left\{R_{k} \leq j<\tau_{k}\right\} \quad \text { since } 1\left\{R_{k} \leq j<\tau_{k}\right\} \geq 0 \\
& =\sum_{j=R_{2} B p_{+1}}^{\tau_{n}} \sum_{k=2^{B p}+1}^{n} 1\left\{R_{k} \leq j<\tau_{k}\right\} \\
& \leq \sum_{j=R_{2} B p_{1}}^{\tau_{n}} \sum_{k=-\infty}^{\infty} 1\left\{R_{k} \leq j<\tau_{k}\right\} \\
& =\sum_{j=R_{2} B_{p}+1}^{\tau_{n}}\left|\left\{k: R_{k} \leq j<\tau_{k}\right\}\right| \\
& \leq \sum_{j=R_{2} B p+1}^{\tau_{n}} 2^{B p} \quad \text { by }(60) \\
& <\sum_{j=\tau_{0}}^{\tau_{n}} 2^{B p} \quad \text { by }(61) \\
& =2^{B p}+2^{B p} \sum_{j=0}^{n-1}\left(\tau_{j+1}-\tau_{j}\right) .
\end{aligned}
$$

We therefore obtain

$$
\begin{aligned}
E_{0}\left(Z_{0}\right) & =\lim _{n \rightarrow \infty} n^{-1}\left[\sum_{k=2^{B p}+1}^{n} Z_{k}\right] \quad P_{0} \text {-a.s. } \\
& \leq 2^{B p} \lim _{n \rightarrow \infty} n^{-1} \sum_{j=0}^{n-1}\left(\tau_{j+1}-\tau_{j}\right) \quad P_{0} \text {-a.s. } \\
& =2^{B p} E_{0}\left(\tau_{1}\right) \quad P_{0} \text {-a.s. } \quad \text { since }\left(P_{0}, \theta_{0}\right) \text { is ergodic } \\
& =2^{B p} \psi^{-1}<\infty . \quad \text { by }(59) .
\end{aligned}
$$




\section{I}




\section{PART II}

\section{The convergence of the norm of the value of $B-$ blocks}

\subsection{Introduction}

We are interested in studying the convergence properties of the asynchronous computation described by equation (1) or equation (4). Recall the assumed bound on the delays, see equation (2). We see that $x(n)$ depends linearly on the components of $x(n-1), \ldots, x(n-$ $B)$. Thus it is possible to construct a $B p$-dimensional system with

$$
y(n)=\left[x^{T}(n) x^{T}(n-1) \ldots x^{T}(n-B+1)\right]^{T} \in \mathbf{R}^{B p},
$$

and a sequence of $B p \times B p$ matrices $A(n)$ satisfying

$$
A(n+1) y(n)=y(n+1) \text { for } n \in \mathbf{Z} .
$$

Note that, in defining $y(n)$, the way we have listed the values of the nodes in the $B$-block $W+n$ is compatible with the lexicographic ordering of the nodes of $W$ that was introduced in Subsection 4.3. The statistical assumptions on the delays make the sequence of matrices $\{A(n), n \in \mathbf{Z}\}$ stationary and ergodic. For any scenario $\omega \in \Omega$, let $M(n, \omega)$ denote $A(n, \omega) \cdots A(1, \omega)$. By Oseledec's multiplicative ergodic theorem, stated as Theorem 1, the number

$$
L(y, \omega)=\lim _{n \rightarrow \infty} n^{-1} \log \|M(n, \omega) y\|,
$$

is well defined for almost all scenarios, and

$$
L(y, \omega) \in\left\{\lambda_{1}, \ldots, \lambda_{s}\right\} \text { if } y \neq 0 .
$$

Here $-\infty \leq \lambda_{s}<\ldots<\lambda_{1}<\infty$, are the nonrandom constants we called the Lyapunov exponents, see Definition 1.

Remark 19 Recall that, until Section 9 all entries of $A$ and all initial conditions are assumed to be nonnegative. Henceforth, $\delta$ will denote $\min \left\{a_{i j}: a_{i j}>0\right\}$. We have $\delta>0$. Also, $\alpha$ will denote $\max _{\omega}\|A(1, \omega)\|$, where $A(n)$ is defined as in equation (63). Since $|\{A(1, \omega): \omega \in \Omega\}| \leq(B)^{p^{2}}$ for any delay function $d$, the max is attained for some $\omega \in \Omega$, and $\alpha<\infty$. 
Given $y, \tilde{y} \in \mathbf{R}_{+}^{B p}$ we say $y \geq \tilde{y}$ if and only if $y_{i} \geq \tilde{y}_{i}$ for all $i$. We say $y>\tilde{y}$ if in addition $y_{j}>\tilde{y}_{j}$ for at least one $j$. It is straightforward to see that

$$
y \geq \tilde{y} \quad \Longrightarrow \quad L(y, \omega) \geq L(\tilde{y}, \omega)
$$

and

$$
L\left(\alpha_{1} y_{1}+\alpha_{2} y_{2}, \omega\right)=\max _{i} L\left(y_{i}, \omega\right) \text { for any } \alpha_{1}, \alpha_{2}>0
$$

Definition 21 (Basic Initial Conditions) The basic initial condition associated to the node $u \in W$ is denoted $e_{u}$, and is the initial condition where the value of node $u$ is set equal to 1, while the values of the other nodes in the base are set equal to 0.

Definition 22 Let $V \subseteq W$ be a subset of the base. We write $\mathbf{R}^{V}$ (resp. $\mathbf{R}_{+}^{V}$ ) for the subspace of $\mathbf{R}^{B p}$ consisting of the vectors (resp. the vectors with nonnegative components) whose components off $V$ are zero. Namely

$$
\begin{aligned}
& \mathbf{R}^{V}=\left\{\sum_{u \in V} a_{u} e_{u}: a_{u} \in \mathbf{R}, u \in V\right\} . \\
& \mathbf{R}_{+}^{V}=\left\{\sum_{u \in V} a_{u} e_{u}: a_{u} \geq 0, u \in V\right\} .
\end{aligned}
$$

From equation (67) we see that the rate of growth of the norms of the vector of values in $B$-blocks for an arbitrary nonnegative initial condition is just the maximum of such rates of growth for each of the basic initial conditions. An immediate consequence is that the set of nonnegative vectors that lie in a Lyapunov subspace is just the direct sum, with nonnegative coefficients, of certain basic initial conditions.

\subsection{Subsets of colors of the spectrum}

For $\omega \in \Omega_{0}$, any basic initial condition $e_{u}$, given by a node $u \in W$, is naturally associated to a subset of the colors of the spectrum, namely those colors that contain it. For any subset of colors of the spectrum, the basic initial conditions to which it corresponds will be the ones associated to nodes that lie in every one of the colors of this subset, and in none of the colors of the complementary subset. These observations motivate the considerations of this Subsection. 
Definition 23 Consider the lexicographic ordering of subsets of the base, introduced in Subsection 4.3. Then, for $\omega \in \Omega_{0}$, we may write $\mathcal{S}(\omega)=\left\{C_{1}(\omega), \ldots, C_{\sigma}(\omega)\right\}$. Given $b \in\{0,1\}^{\sigma}$, we write $\mathcal{S}(b, \omega)$ for $\left\{C_{i}(\omega): b_{i}=1\right\}$,

Definition 24 For $\omega \in \Omega_{0}, n \in \mathbf{Z}$, and any subset of colors $\mathcal{S} \subseteq \mathcal{S}(\omega)$, we write $\mathcal{I}(n, \mathcal{S}, \omega)$ for $\bigcap_{C \in \mathcal{S}} \theta^{n} C \backslash \bigcup_{C \in \mathcal{S}(\omega) \backslash \mathcal{S}} \theta^{n} C$.

Definition 25 For $b \in\{0,1\}^{\sigma}$ and $\pi \in \mathcal{P}_{\sigma}$ we let $\pi[b] \in\{0,1\}^{\sigma}$ be defined by

$$
\pi[b]_{j}=1 \text { if and only if } b_{\pi^{-1}[j]}=1 .
$$

Recall the definition of the random permutation $\pi(\omega) \in \mathcal{P}_{\sigma}$, in equation (28). Then we have the following result

Lemma 18 For all $\omega \in \Omega_{0}$, for all $b \in\{0,1\}^{\sigma}$,

$$
\theta \mathcal{S}(b, \omega)=\mathcal{S}(\pi(\omega)[b], \theta \omega), \text { and }
$$

$$
\mathcal{I}(n, \mathcal{S}(b, \omega), \omega)=\mathcal{I}(n-1, \mathcal{S}(\pi(\omega)[b], \theta \omega), \theta \omega) \text { for all } n \in \mathbf{Z}
$$

Proof : Let $\omega \in \Omega_{0}$. To prove (1), we write

$$
\begin{aligned}
\theta \mathcal{S}(b, \omega) & =\theta\left\{C_{i}(\omega): b_{i}=1\right\} \\
& =\left\{\theta C_{i}(\omega): b_{i}=1\right\} \\
& =\left\{C_{\pi(\omega)[i]}(\theta \omega): b_{i}=1\right\} \\
& =\left\{C_{i}(\theta \omega): b_{\pi(\omega)^{-1}[i]}=1\right\} \\
& =\mathcal{S}(\pi(\omega)[b], \theta \omega)
\end{aligned}
$$

where we go from the second line to the third line using equation (29). This proves (1).

To prove part (2) of the lemma, we write, for $\omega \in \Omega_{0}$ and $n \in \mathbf{Z}$,

$$
\begin{aligned}
\mathcal{I}(n, \mathcal{S}(b, \omega), \omega) & =\bigcap_{C \in \mathcal{S}(b, \omega)} \theta^{n} C \backslash \cup_{C \in \mathcal{S}(\omega) \backslash \mathcal{S}(b, \omega)} \theta^{n} C \\
& =\cap_{C \in \theta \mathcal{S}(b, \omega)} \theta^{n-1} C \backslash \cup_{C \in \mathcal{S}(\theta \omega) \backslash \theta \mathcal{S}(b, \omega)} \theta^{n-1} C \\
& =\mathcal{I}(n-1, \theta \mathcal{S}(b, \omega), \theta \omega) \\
& =\mathcal{I}(n-1, \mathcal{S}(\pi(\omega)[b], \theta \omega), \theta \omega)
\end{aligned}
$$

where the last step is by part (1) of the lemma. This proves the lemma. 


\subsection{Associating modes to subsets of colors}

The main result of this section is that, if $A$ is a nonnegative matrix and the initial conditions are nonnegative, it is possible to associate modes to subsets of colors in an invariant way. The final form of this result is presented later as Theorem 6, but is essentially based on the following result.

Proposition 7 Let $A$ be positive. Then, for an a.s.s.i. subset of scenarios the following hold.

(1) If $\mathcal{I}(n, \mathcal{S}(b, \omega), \omega) \neq \emptyset$ for some $n$, then $\mathcal{I}(n, \mathcal{S}(b, \omega), \omega) \neq \emptyset$ for infinitely many $n$.

(2) Moreover, if $n_{1}<n_{2}$ are such that $\mathcal{I}\left(n_{i}, \mathcal{S}(b, \omega), \omega\right) \neq \emptyset$, for $i=1,2$, then:

(2a) For all $y \in \mathbf{R}_{+}^{\mathcal{I}\left(n_{1}, \mathcal{S}(b, \omega), \omega\right)}, y \neq 0$, we have $L\left(y, \theta^{n_{1}} \omega\right)=\lambda \in\left\{\lambda_{1}, \ldots, \lambda_{s}\right\}$ with $\lambda>-\infty$. Further, $\lambda$ is the same for all such $y$.

(2b) For all $y \in \mathbf{R}_{+}^{\mathcal{I}\left(n_{2}, \mathcal{S}(b, \omega), \omega\right)}, y \neq 0$, we have $L\left(y, \theta^{n_{2}} \omega\right)=\lambda$. This is the same $\lambda$ as the one in part (2a), and is also the same for all such $y$.

Proof : Let $\omega \in \Omega_{0}$. The claim (1) is a special case of the result of Lemma 7, given by taking $\mathcal{C}_{1}=\mathcal{S}(b, \omega)$ and $\mathcal{C}_{2}=\mathcal{S}(\omega) \backslash \mathcal{S}(b, \omega)$ in that lemma.

To prove part (2) of the lemma, let $n_{1}<n_{2}$ be such that $\mathcal{I}\left(n_{i}, \mathcal{S}(b, \omega), \omega\right) \neq \emptyset$ and let $\omega_{1}=\theta^{n_{1}} \omega$, and $\mathcal{S}=\theta^{n_{1}} \mathcal{S}(b, \omega)$. Let $u \in \mathcal{I}\left(n_{1}, \mathcal{S}(b, \omega), \omega\right)$ and set $y(0)=e_{u}$. Since $u \in C$ for some $C \in \mathcal{S} \subseteq \mathcal{S}\left(\omega_{1}\right)$, in each B-block $W+n$, there exists a node $\left(q_{n}, m_{n}\right)$ with $u$ in its history, and hence $x_{q_{n}}\left(m_{n}, \omega_{1}\right) \geq \min \left(\delta^{n}, \delta^{\lfloor n / B\rfloor}\right)$. In view of equation (67) this proves that $L\left(y, \omega_{1}\right)>-\infty$ for all $y \in \mathbf{R}_{+}^{\mathcal{I}\left(n_{1}, \mathcal{S}(b, \omega), \omega\right)}, y \neq 0$. If such $u$ is unique, this proves part (2a) of the proposition.

If such $u$ is not unique, assume $u_{1}, u_{2} \in \mathcal{I}\left(n_{1}, \mathcal{S}(b, \omega), \omega\right), u_{1} \neq u_{2}$. Let $N_{1}$ denote the random time $N\left(\omega_{1}\right)$, defined in equation (45). We have $C\left(v, \omega_{1}\right) \in \mathcal{S}\left(\omega_{1}\right)$ whenever $T(v) \geq N_{1}$. Therefore, whether we set $y(0)=e_{u_{1}}$ or set $y(0)=e_{u_{2}}$, exactly the same set of nodes in $W+N_{1}+B$ will have nonzero (positive) values, namely those nodes with color in $\mathcal{S}$, (and thus with both $u_{1}$ and $u_{2}$ in their histories), as all other nodes in $W+N_{1}+B$ have colors in $\mathcal{S}(\omega) \backslash \mathcal{S}$ and hence will have value zero. So if we let $y^{i}=M\left(N_{1}+B, \omega_{1}\right) e_{u_{i}}$, exactly the same components of $y^{1}$ and $y^{2}$ will be positive (with the rest zero). Now define

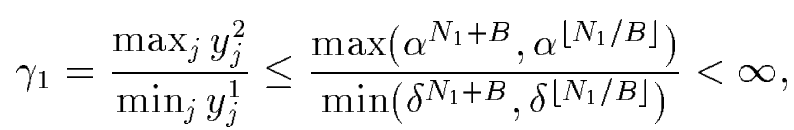


where the max and min are over the nonzero components of $y^{i}$. Similarly, define $\gamma_{2}<\infty$ as $\gamma_{1}$ but with superscripts 1 and 2 interchanged. Since exactly the same components of $y^{1}$ and $y^{2}$ are nonzero (and positive) we get

$$
\gamma_{1}^{-1} y^{2} \leq y^{1} \leq \gamma_{2} y^{2}
$$

and since

$$
L\left(e_{u_{i}}, \omega_{1}\right)=\lim n^{-1} \log \left\|A\left(n, \omega_{1}\right) \cdots A\left(N_{1}+B+1, \omega_{1}\right) y^{i}\right\|,
$$

by equations (66) and (67) we get

$$
L\left(e_{u_{2}}, \omega_{1}\right)=L\left(\gamma_{1}^{-1} e_{u_{2}}, \omega_{1}\right) \leq L\left(e_{u_{1}}, \omega_{1}\right) \leq L\left(\gamma_{2} e_{u_{2}}, \omega_{1}\right)=L\left(e_{u_{2}}, \omega_{1}\right)
$$

That is, $L\left(e_{u_{1}}, \omega_{1}\right)=L\left(e_{u_{2}}, \omega_{1}\right)$ for all $u_{1}, u_{2} \in \mathcal{I}\left(n_{1}, \mathcal{S}(b, \omega), \omega\right)$, which in view of equation (67) completes the proof of part (2a) of the proposition.

To prove part (2b) of the proposition, let $\omega_{2}=\theta^{n_{2}} \omega=\theta^{n_{2}-n_{1}} \omega_{1}$. Let $N_{2}$ denote $N\left(\omega_{2}\right)$ defined as in equation (45). For a node $v$ with $T(v) \geq N_{2}$, we then have $C\left(v, \omega_{2}\right) \in \mathcal{S}\left(\omega_{2}\right)$. Further, by equation (18), we would also have $C\left(v+n_{2}-n_{1}, \omega_{1}\right)=\theta^{n_{2}-n_{1}} C\left(v, \omega_{2}\right) \in$ $\mathcal{S}\left(\omega_{1}\right)$. Now pick $y \in \mathbf{R}_{+}^{\mathcal{I}\left(n_{1}, \mathcal{S}(b, \omega), \omega\right)}, y \neq 0$, and $y^{\prime} \in \mathbf{R}_{+}^{\mathcal{I}\left(n_{2}, \mathcal{S}(b, \omega), \omega\right)}, y^{\prime} \neq 0$, and let $y^{1}=M\left(N_{2}+B+n_{2}-n_{1}, \omega_{1}\right) y$ and $y^{2}=M\left(N_{2}+B, \omega_{2}\right) y^{\prime}$. Then $y^{1}$ and $y^{2}$ have exactly the same nonzero (positive) components. This may be seen as follows : the entries of $y^{1}$ are being computed as values of nodes of the $B$-block $W+N_{2}+B+n_{2}-n_{1}$ relative to initial conditions in $W$, at scenario $\omega_{1}$. Because $C\left(v+n_{2}-n_{1}, \omega_{1}\right) \in \mathcal{S}\left(\omega_{1}\right)$ for every node $v$ with $T(v) \geq N_{2}$, the nonzero components of $y^{1}$ will be precisely those corresponding to nodes having color in $\mathcal{S}$. But we see that the entries of $y^{1}$ could equally well be computed as values of nodes in the $B$-block $W+N_{2}+B+n_{2}$ relative to initial conditions in the $B$ block $W+n_{1}$ at scenario $\omega$, in which case the nonzero components of $y^{1}$ will be precisely those corresponding to nodes with colors in $\mathcal{S}(b, \omega)=\left\{C_{i}(\omega): b_{i}=1\right\}$, using equation (18). An analogous argument shows that the nonzero components of $y^{2}$ are also precisely those corresponding to nodes with colors in $\mathcal{S}(b, \omega)=\left\{C_{i}(\omega): b_{i}=1\right\}$. This now implies the existence of $\beta_{1}, \beta_{2}<\infty$ such that

$$
\beta_{1}^{-1} y^{1} \leq y^{2} \leq \beta_{2} y^{1}
$$

The rest of the proof is similar to that of part $(2 \mathrm{a})$ once we note that

$$
L\left(y, \omega_{1}\right)=\lim n^{-1} \log \left\|A\left(n, \omega_{1}\right) \cdots A\left(N_{2}+B+n_{2}-n_{1}+1, \omega_{1}\right) y^{1}\right\|
$$




$$
\begin{gathered}
\operatorname{and} L\left(y^{\prime}, \omega_{2}\right)=\lim n^{-1} \log \left\|A\left(n, \omega_{2}\right) \cdots A\left(N_{2}+B+1, \omega_{2}\right) y^{2}\right\| \\
=\lim n^{-1} \log \left\|A\left(n+n_{2}-n_{1}, \omega_{1}\right) \cdots A\left(N_{2}+B+n_{2}-n_{1}+1, \omega_{1}\right) y^{2}\right\| .
\end{gathered}
$$

Remark 20 From now on, $\Omega_{0}$ will denote an a.s.s.i. subset of scenarios for which Proposition 7 holds. Without loss of generality, we will assume that all preceding results hold for all scenarios in $\Omega_{0}$,

\subsection{Invariance of the modal decomposition}

Let $\mathcal{L}$ denote the collection of all maps from $\{0,1\}^{\sigma}$ to $\left\{\lambda_{1}, \ldots, \lambda_{s}, \phi\right\}$, where $\phi$ is a symbol. An element of $\mathcal{L}$ can be thought of as a marking on the lattice of subsets of $\{1, \ldots, \sigma\}$. In view of proposition 7 , the following definition makes sense :

Definition 26 For $\omega \in \Omega_{0}$, define $\Lambda(\omega):\{0,1\}^{\sigma} \rightarrow\left\{\lambda_{1}, \ldots, \lambda_{s}, \phi\right\}$ by

$$
\Lambda(\omega)(b)=\left\{\begin{array}{rc}
L\left(y, \theta^{n} \omega\right) \quad & \text { for any } y \in \mathbf{R}_{+}^{\mathcal{I}(n, \mathcal{S}(b, \omega), \omega)}, y \neq 0 \\
\phi, & \text { if } \mathcal{I}(n, \mathcal{S}(b, \omega), \omega) \neq \emptyset \text { for some } n \in \mathbf{Z} \\
& \text { if } \mathcal{I}(n, \mathcal{S}, \omega)=\emptyset \text { for all } n \in \mathbf{Z} .
\end{array}\right.
$$

Then, for all $\omega \in \Omega_{0}, \Lambda(\omega)$ as defined above is a member of $\mathcal{L}$. $\phi$ can be thought of as a symbol indicating that certain subsets of the spectrum do not correspond to any basic initial condition. We next identify elements of $\mathcal{L}$ that differ only by a permutation of $\{1, \ldots, \sigma\}$. Given any $\Lambda \in \mathcal{L}$ define $[\Lambda]$ to be the corresponding equivalence class. The main result of this section can now be expressed as follows :

Theorem 6 There exists a unique equivalence class $\left[\Lambda^{*}\right]$ of markings on the lattice of subsets of $\{1, \ldots, \sigma\}$ by marks in $\left\{\lambda_{1}, \ldots, \lambda_{s}, \phi\right\}$ such that for an a.s.s.i. subset of scenarios we have $[\Lambda(\omega)]=\left[\Lambda^{*}\right]$.

Proof : Let $\omega \in \Omega_{0}$. We first claim that $[\Lambda(\theta \omega)]=[\Lambda(\omega)]$. To see this it suffices to note that, by part (2) of Lemma 18 , for any $b \in\{0,1\}^{\sigma}$ we have

$$
\mathcal{I}\left(n+1, \mathcal{S}\left(\pi(\omega)^{-1}[b], \omega\right), \omega\right)=\mathcal{I}(n, \mathcal{S}(b, \theta \omega), \theta \omega)
$$


Now, by Definition 26, $\Lambda(\theta \omega)(b)=\phi$ if and only if $\mathcal{I}(n, \mathcal{S}(b, \theta \omega), \theta \omega)=\emptyset$ for all $n \in \mathbf{Z}$. By equation (74) this is true if and only if $\mathcal{I}\left(n+1, \mathcal{S}\left(\pi(\omega)^{-1}[b], \omega\right), \omega\right)=\emptyset$ for all $n \in \mathbf{Z}$, which, by Definition 26 again, is true if and only if $\Lambda(\omega)\left(\pi(\omega)^{-1}[b]\right)=\phi$. Similarly, by Definition 26, $\Lambda(\theta \omega)(b)=\lambda \in\left\{\lambda_{1}, \ldots, \lambda_{s}\right\}$ if and only if $\mathcal{I}(n, \mathcal{S}(b, \theta \omega), \theta \omega) \neq \emptyset$ for some $n \in \mathbf{Z}$, and, for some $y \in \mathbf{R}_{+}^{\mathcal{I}(n, \mathcal{S}(b, \theta \omega), \theta \omega)}, y \neq 0$, we have $L\left(y, \theta^{n} \theta \omega\right)=\lambda$. But, by equation (74), the very same $y$ is then in $\mathcal{I}\left(n+1, \mathcal{S}\left(\pi(\omega)^{-1}[b], \omega\right), \omega\right)$ and we have $L\left(y, \theta^{n+1} \omega\right)=\lambda$, so by Definition 26 we have $\Lambda(\omega)\left(\pi(\omega)^{-1}[b]\right)=\lambda$. We have proved that $[\Lambda(\theta \omega)]=[\Lambda(\omega)]$.

We get a partition of $\Omega_{0}$ with elements

$$
\Omega_{[\Lambda]}=\left\{\omega \in \Omega_{0}:[\Lambda(\omega)]=[\Lambda]\right\}
$$

What we just proved is that $\theta \Omega_{[\Lambda]} \subseteq \Omega_{[\Lambda]}$. Since there are only finitely many equivalence classes in $\mathcal{L}$, and since $\Omega_{0}$ has probability 1 , it is now immediate from ergodicity that there is one and only one equivalence class $\left[\Lambda^{*}\right]$ such that $P\left(\Omega_{\left[\Lambda^{*}\right]}\right)=1$. This completes the proof of the theorem.

\subsection{An example}

We illustrate the concepts and results of this section by means of a simple example. Consider the example of Subsection 2.4. With our lexicographic ordering convention of Subsection 4.3 and the notation introduced in subsection 2.4 we have

$$
C_{1}\left(\omega_{0}\right)=C_{2}, C_{2}\left(\omega_{0}\right)=C_{1}, C_{1}\left(\omega_{1}\right)=C_{2}^{\prime}, \text { and } C_{2}\left(\omega_{1}\right)=C_{1}^{\prime}
$$

Note that $\theta C_{1}\left(\omega_{0}\right)=C_{1}\left(\omega_{1}\right)$. Thus $\pi\left(\omega_{0}\right)$ as defined in subsection 4.3 is the identity permutation.

We now calculate the marking $\Lambda\left(\omega_{0}\right)$. As always, $\Lambda\left(\omega_{0}\right)(00)=\phi$. Note that

$$
\mathcal{I}\left(0, \mathcal{S}\left(10, \omega_{0}\right), \omega_{0}\right)=C_{2} \backslash C_{1}=\{(2,-1)\} \neq \emptyset
$$

Thus $\Lambda\left(\omega_{0}\right)(10)$ equals some Lyapunov exponent $\lambda_{\alpha}$. To evaluate $\Lambda\left(\omega_{0}\right)(01)$ note that

$$
\begin{aligned}
& \mathcal{I}\left(n, \mathcal{S}\left(01, \omega_{0}\right), \omega_{0}\right)=\theta^{n} C_{2}\left(\omega_{0}\right) \backslash \theta^{n} C_{1}\left(\omega_{0}\right) \\
& =\left\{\begin{array}{l}
C_{1} \backslash C_{2} \text { if } n \text { is even } \\
C_{1}^{\prime} \backslash C_{2}^{\prime} \text { if } n \text { is odd . }
\end{array}\right. \\
& =\emptyset \quad \text { for all } n \text {. }
\end{aligned}
$$

Thus $\Lambda\left(\omega_{0}\right)(01)=\phi$. To evaluate $\Lambda\left(\omega_{0}\right)(11)$ we observe that $\mathcal{I}\left(0, \mathcal{S}\left(11, \omega_{0}\right), \omega_{0}\right)=C_{1} \neq \emptyset$. Thus $\Lambda\left(\omega_{0}\right)(11)=\lambda_{\beta}$ for some Lyapunov exponent $\lambda_{\beta}$. 
We may similarly determine $\Lambda\left(\omega_{1}\right)$, and find that

$$
\Lambda\left(\omega_{1}\right)(00)=\phi, \Lambda\left(\omega_{1}\right)(10)=\lambda_{\alpha}, \Lambda\left(\omega_{1}\right)(01)=\phi, \Lambda\left(\omega_{1}\right)(11)=\lambda_{\beta}
$$

Note that $\left[\Lambda\left(\omega_{1}\right)\right]=\left[\Lambda\left(\omega_{0}\right)\right]$.

\section{Convergence rate of sequences of processor-time values}

\subsection{A sequence of random invariant subspaces}

Let $\omega \in \Omega_{0}$. Recall the sets $\mathcal{S}_{i}(\omega), 1 \leq i \leq \rho$, which were introduced in Definition 11. We now show that these sets naturally induce a nested family of random subspaces of $\mathbf{R}^{B p}$.

Definition 27 For $\omega \in \Omega_{0}$, for $i=1, \ldots, \rho$, define

$$
U_{i}(\omega)=\bigcup_{j=1}^{i} \bigcup_{C \in \mathcal{S}_{j}(\omega)} F_{C}(\omega) .
$$

Note that this is a nested sequence of sets. Let $\bar{U}_{i}(\omega)=U \backslash U_{i}(\omega)$.

Let

$$
U_{i}(n, \omega)=U_{i}(\omega) \cap(W+n)-n, \bar{U}_{i}(n, \omega)=\bar{U}_{i}(\omega) \cap(W+n)-n=W \backslash U_{i}(n, \omega)
$$

Note that by equations (23) and (34), we have

$$
U_{i}(n, \theta \omega)=U_{i}(n+1, \omega), \quad n \in \mathbf{Z},
$$

Further, by part (3) of Lemma 1, if $\langle u, v\rangle \in E(\omega)$ for some $v \in U_{i}(\omega)$ then $u \in U_{i}(\omega)$, so we have

$$
A(n+1, \omega) \mathbf{R}^{\bar{U}_{i}(n, \omega)} \subseteq \mathbf{R}^{\bar{U}_{i}(n+1, \omega)}, \quad n \in \mathbf{Z}
$$

where we recall that $A(n)$ is defined in equation (63).

Equation (77) motivates the following definition :

Definition 28 For $\omega \in \Omega_{0}$ and $n \in \mathbf{Z}$, define

$U_{1}^{*}(n, \omega)=U_{1}(n, \omega), \quad U_{i}^{*}(n, \omega)=U_{i}(n, \omega) \backslash U_{i-1}(n, \omega)$ for $i=2, \ldots, \rho, \quad$ and $U_{\rho+1}^{*}(n, \omega)=\bar{U}_{\rho}(n, \omega)$. 
From equation (77) we have, for all $n \in \mathbf{Z}$ and all $1 \leq i \leq \rho+1$,

$$
A(n+1, \omega) \mathbf{R}_{i}^{U_{i}^{*}(n, \omega)} \subseteq \oplus_{j=i}^{\rho+1} \mathbf{R}^{U_{j}^{*}(n+1, \omega)} .
$$

\subsection{A decomposition of $A(n)$}

The nested random subspaces of $\mathbf{R}^{B p}$ introduced in Definition 28 lead to a lower triangular decomposition of the matrix $A(n+1, \omega)$. Indeed, from equation (79) we may immediately write

$$
A(n+1)=\sum_{i=1}^{\rho+1} \sum_{j=1}^{i} A_{i j}(n+1),
$$

where $A_{i j}(n+1): \mathbf{R}^{B p} \rightarrow \mathbf{R}^{B p}$ are uniquely defined by

$$
\left[A_{i j}\right]_{u v}(n+1)= \begin{cases}A_{u v}(n+1) & \text { if } v \in U_{j}^{*}(n, \omega) \text { and } u \in U_{i}^{*}(n+1, \omega), \\ 0 & \text { otherwise. }\end{cases}
$$

By definition $A(n, \theta \omega)=A(n+1, \omega)$, so by virtue of equation (76), we see that

$$
A_{i j}(n, \theta \omega)=A_{i j}(n+1, \omega)
$$

as well. Therefore, for $i=1, \ldots, \rho+1,\left\{A_{i i}(n), n \in \mathbf{Z}\right\}$ is a stationary ergodic sequence of $B p \times B p$ matrices. Since $\left\|A_{i i}(1)\right\| \leq\|A(1)\|$ for all $i$, we can apply OMET, see Theorem 1 , endowing this sequence of random matrices with its own set of Lyapunov exponents and Oseledec subspaces.

Definition 29 For $i=1, \ldots, \rho+1$, define $\Lambda_{i}$ to be the set of Lyapunov exponents of sequence $\left\{A_{i i}(n), n \in \mathbf{Z}\right\}$.

Definition 30 Let $U_{0} \subseteq U$ be an arbitrary subset of nodes. Let $(y(n), n \geq 0)$ be $\mathbf{R}^{B p}$ valued vectors resulting from a computation started with initial conditions $y(0)$ for the nodes in $W$, defined as in equation (62). We will write $y^{U_{0}}(n)$ for the $\mathbf{R}^{B p}$-valued vector resulting from $y(n)$ by setting the values corresponding to the nodes that are not in $U_{0} \cap$ $(W+n)$ equal to zero. If $u \in W+n$ we will write $y_{u}(n)$ for the value of $y(n)$ in the coordinate corresponding to node $u$.

Definition 30 explains expressions like $y_{u_{k}}\left(n_{k}\right)$ and $y^{F_{C}}(n)$, which show up in the statement and proof of the main theorem in the subsequent subsections 


\subsection{Main theorem}

The following theorem is the main result of the paper :

Theorem 7 The following are true in an a.s.s.i. subset of scenarios :

(1) To each pure color $C \in \mathcal{S}(\omega)$ there is an associated number $\lambda_{C}$ such that $\lambda_{C} \in \Lambda_{i}$ if $C \in \mathcal{S}_{i}(\omega)$, and $\lambda_{C}>-\infty$. Further, $\lambda_{C}(\omega)=\lambda_{\theta C}(\theta \omega)$.

(2) Let $C \in \mathcal{S}(\omega)$ be an arbitrary color. If the computation is started in the basic initial condition corresponding to $u \in W$, and if $\left\{n_{k}\right\}_{k=1}^{\infty}$ is an increasing sequence such that every $W+n_{k}$ contains at least one $C$-colored node, then for any sequence $u_{k} \in W+n_{k}$ of $C$-colored nodes, the limit

$$
\lim _{k \rightarrow \infty} n_{k}^{-1} \log y_{u_{k}}\left(n_{k}\right)=\lambda(C, u)
$$

exists. Further, this limit is the same for all such sequences and can be computed as follows:

(i) If $u \notin C$, then $\lambda(C, u)=-\infty$.

(ii) If $C$ is pure and $u \in C$, then

$$
\lambda(C, u)=\max \left\{\lambda_{C}, \max \left\{\lambda\left(C^{\prime}, u\right): C^{\prime} \subseteq C\right\}\right\} .
$$

(iii) If $C$ is not pure and $u \in C$, then

$$
\lambda(C, u)=\max \left\{\lambda\left(C^{\prime}, u\right): C^{\prime} \subseteq C\right\} .
$$

(3) For an arbitrary positive initial condition $y(0)$ for nodes in the base $W$, for $C \in$ $\mathcal{S}(\omega)$, and for an increasing sequence $\left\{n_{k}\right\}_{k=1}^{\infty}$, and a $C$-colored sequence of nodes $u_{k} \in$ $W+n_{k}$ as above, the limit

$$
\lim _{k \rightarrow \infty} n_{k}^{-1} \log y_{u_{k}}\left(n_{k}\right)=\lambda(C, y)
$$

exists and can be computed as

$$
\lambda(C, y)=\max _{u \in W: y_{u}>0} \lambda(C, u)
$$

(4) Given an arbitrary indexing of the $\sigma$ colors of the spectrum and the partial order on $\{1, \ldots, \sigma\}$ induced by the inclusion partial order on colors, consider the marked partial 
order where each pure color is marked by its corresponding $\lambda_{C}$ and the non-pure colors are marked by an arbitrary symbol. Let $\Xi^{\lambda}$ denote the equivalence class of this marked partial order up to reindexing of the colors, thought of as a random variable. Then this random variable is a.s. constant.

Remark 21 There are examples where one has a color of the spectrum that does not show up in infinitely many $B$-blocks. This explains why the statement of part (2) of Theorem 7 is the way it is. Here is such an example. Let $A$ be a $2 \times 2$ matrix with no zero elements. Let $B=3$ and $\Omega=\left\{\omega_{0}, \ldots, \omega_{5}\right\}$ with $P\left(\omega_{i}\right)=1 / 6$ and $\theta \omega_{i}=\omega_{(i+1) \bmod 6 \text {. }}$ Then $(P, \theta)$ is stationary and ergodic. Let $d\left(n, \omega_{i}\right)=d\left(n \bmod 6, \omega_{i}\right)$ (i.e. the delays are periodic with period 6), with

$$
\begin{gathered}
d\left(1, \omega_{0}\right)=d\left(2, \omega_{0}\right)=d\left(4, \omega_{0}\right)=d\left(5, \omega_{0}\right)=\left(\begin{array}{ll}
3 & 3 \\
3 & 3
\end{array}\right), \\
d\left(3, \omega_{0}\right)=\left(\begin{array}{ll}
3 & 1 \\
1 & 2
\end{array}\right), \quad d\left(6, \omega_{0}\right)=\left(\begin{array}{ll}
3 & 2 \\
1 & 1
\end{array}\right) .
\end{gathered}
$$

To make $d$ compatible with $\theta$, we let $d\left(n, \omega_{(i+1) \bmod 6}\right)=d\left((n+1) \bmod 6, \omega_{i}\right)$.

Using the periodicity of the delays, one can show that $\mathcal{S}\left(\omega_{0}\right)=\left\{C_{a}, C_{b}, C_{c}, C_{d}\right\}$, where $C_{a}=\{(1,-2),(2,-2)\}, C_{b}=\{(1,-1),(2,-1)\}, C_{c}=C_{a} \cup C_{b}$, and $C_{d}=C_{c} \cup\{(1,0)\}$. Then, for example $W+6 n, n \geq 1$, contain no $C_{c}$-colored nodes. Note that $C_{c}$ is not pure. In fact, one can show that for every pure color, in all but finitely many nonnegative $B$-blocks, there must be a node having that color. To see this, let $C$ be a pure color. First pick $n \geq B$ large enough so that every node in $W+n$ has a color in $\mathcal{S}(\omega)$, i.e. choose $n \geq N(\omega)+B-1$ in the notation of equation (45). Let $m \geq n+B$ be such that $W+n$ contains a node of color $C$, call it $w$. Such $m$ must exist, because $C$, being a color of the spectrum, shows up as the color of infinitely many nodes at scenario $\omega$. List the nodes in $W+n$ that influence $w$ as $\left\{v_{i}, a \in \mathcal{A}\right\}$, and let $C_{a}$ denote $C\left(v_{a}, \omega\right)$, so that $C_{a} \in \mathcal{S}(\omega)$ for all $a \in \mathcal{A}$. We also have $C_{a} \subseteq C$ for all $a \in \mathcal{A}$ and $\cup_{a \in \mathcal{A}} C_{a}=C$. It follows that at least one of the $C_{a}$ must equal $C$, otherwise we would have $C=\cup_{i \in \mathcal{S}_{C}} C_{i}$, contradicting the purity of $C$. Thus we have shown that every $B$-block $W+n$ with $n \geq N(\omega)+B-1$ must contain a node of color $C$.

We now sketch how we prove Theorem 7 . It suffices to prove parts (1) and (2) of the theorem. Indeed, once this is done, part (3) of the theorem is obvious from the positivity 
of the matrix and the initial conditions, which has been assumed throughout. Part (4) of the theorem is a direct consequence of the invariance $\lambda_{C}(\omega)=\lambda_{\theta C}(\omega)$, equation (39), and a standard ergodicity argument along the lines used several times before, see the proofs of Theorem 3 and Proposition 6.

The proof of parts (1) and (2) of the theorem is broken into two stages. In the first stage, which is the content of Subsection 8.4, we will show that, almost surely, if for some $C \in \mathcal{S}(\omega)$ and an initial condition $y(0)$ for the nodes of the base, we have that $\lim _{n \rightarrow \infty} n^{-1} \log \left\|y^{F_{C}}(n)\right\|$ exists, then for any sequence $u_{k} \in W+n_{k}$ of $C$-colored nodes as in the statement of part (2) of Theorem 7 , we have

$$
\lim _{k \rightarrow \infty} n_{k}^{-1} \log y_{u_{k}}\left(n_{k}\right) \text { exists and equals } \lim _{n \rightarrow \infty} n^{-1} \log \left\|y^{F_{C}}(n)\right\|
$$

In the second stage, which is the content of Subsection 8.5, we will prove that for all $C \in \mathcal{S}(\omega), \lim _{n \rightarrow \infty} n^{-1} \log \left\|y^{F_{C}}(n)\right\|$ exists for any basic initial condition $y(0)=e_{u}$. We will also show that there are exponents $\lambda_{C}(\omega)$ for pure $C \in \mathcal{S}(\omega)$ as described in part (1) of Theorem 7 and such that if we denote $\lim _{n \rightarrow \infty} n^{-1} \log \left\|y^{F_{C}}(n)\right\|$ by $\lambda(C, u)$, then $\lambda(C, u)$ can be described in terms of these $\lambda_{C}, C$ pure, as in part (2) of the Theorem 7.

\subsection{The first half of the proof of the main theorem}

In this section we carry out the proof of the first half of Theorem 7 along the lines of the sketch at the end of the previous subsection. Theorem 5 implies that for any $\epsilon>0$ we have $\sum_{i=1}^{\infty} P_{0}\left(Z_{i}>i \epsilon\right)<\infty$. By the Borel-Cantelli Lemma, this implies that $Z_{i} / i \rightarrow 0$ $P_{0}$-a.s.. But then, by (7.1.2) of [1] we get $\lim _{i \rightarrow \infty} Z_{i} / i=0$-a.s., which by equation (53) implies $\lim _{n \rightarrow \infty} Z_{\nu(n)} / \nu(n)=0$ P-a.s. Using the fact that $\nu(n) \leq n+1$ for all $n>0$, this leads us to:

$$
\lim _{n \rightarrow \infty} Z_{\nu(n)} / n=0 \quad P \text {-a.s. }
$$

Remark 22 From now on, let $\Omega_{0}$ denote an a.s.s.i. subset of scenarios on which the convergence in equation (84) holds. Without loss of generality, we will assume that all previously proved results hold for every scenario in $\Omega_{0}$.

The main result of this subsection is the following :

Proposition 8 Let $\omega \in \Omega_{0}$. For $C \in \mathcal{S}(\omega)$, let $\left\{n_{k}\right\}_{k=1}^{\infty}$ be an increasing sequence such that every $W+n_{k}$ contains at least one $C$-colored node. Pick any sequence of $C$-colored 
nodes $u_{k} \in W+n_{k}$. Suppose $\lim _{n \rightarrow \infty} n^{-1} \log \left\|y^{F_{C}}(n)\right\|$ exists and equals $\zeta \in \mathbf{R} \cup\{-\infty\}$. Then we have

$$
\lim _{k \rightarrow \infty} n_{k}{ }^{-1} \log y_{u_{k}}\left(n_{k}\right)=\zeta
$$

Proof : If $n_{k}$ is large enough, $C\left(u_{k}, \omega\right) \in \mathcal{S}(\omega)$. So $\hat{C}\left(u_{k}, \omega\right)=C\left(u_{k}, \omega\right)=C$. Since $u_{k} \in W+n_{k}$, we have $T\left(u_{k}\right) \geq n_{k}-B$. By equation (51) for all large enough $k$ we have $\beta\left(n_{k}-B\right)>B-1$. By equation (49) we have

$$
\begin{aligned}
C\left(u_{k}-\beta\left(n_{k}-B\right), \theta^{\beta\left(n_{k}-B\right)} \omega\right) & =\theta^{\beta\left(n_{k}-B\right)} \hat{C}\left(u_{k}, \omega\right) \\
& =\theta^{\beta\left(n_{k}-B\right)} C .
\end{aligned}
$$

According to the interpretation of extended color in Subsection 6.2, the values of nodes in $\theta^{\beta\left(n_{k}-B\right)} C+\beta\left(n_{k}-B\right)$ completely determine the value of node $u_{k}$, and $u_{k}$ is influenced by every one of these. By equation (25) it follows that the values at the nodes in $F_{C}(\omega) \cap\left(W+\beta\left(n_{k}-B\right)\right)$ completely determine the value of node $u_{k}$. Note that since $\beta\left(n_{k}-B\right)-B+1>0$, every such node has a positive time index. Since the number of paths from nodes in $F_{C}(\omega) \cap\left(W+\beta\left(n_{k}-B\right)\right)$ to $u_{k}$ is bounded by $\left(p^{2}\right)^{n_{k}-\beta\left(n_{k}-B\right)+B-1}$ and each path is of length at most $n_{k}-\beta\left(n_{k}-B\right)+B-1$, we get the upper bound

$$
y_{u_{k}}\left(n_{k}\right) \leq\left(\gamma p^{2}\right)^{n_{k}-\beta\left(n_{k}-B\right)+B-1}\left\|y^{F_{C}}\left(\beta\left(n_{k}-B\right)\right)\right\|
$$

where $\gamma=\max _{i j} A_{i j}$.

Similarly, since there is a path to $u_{k}$ from the node in $F_{C}(\omega) \cap\left(W+\beta\left(n_{k}-B\right)\right)$ having the largest absolute value, and this is true even if this node is not in $\theta^{\beta\left(n_{k}-B\right)} C$, we have the lower bound

$$
y_{u_{k}}\left(n_{k}\right) \geq \min (1, \delta)^{\left\lceil n_{k}-B-\beta\left(n_{k}-B\right)\right\rceil}\left\|y^{F_{C}}\left(\beta\left(n_{k}-B\right)\right)\right\|
$$

Taking logarithms on both sides, the claim will follow if

$$
\lim _{k \rightarrow \infty} n_{k}^{-1}\left(n_{k}-B-\beta\left(n_{k}-B\right)\right)=0 \quad P \text {-a.s.. }
$$

By equation (54) we have $Z_{\nu\left(n_{k}-B\right)} \geq n_{k}-B-\beta\left(n_{k}-B\right)$, so equation (85) follows from equation (84). 


\subsection{Second half of the proof of the Main Theorem}

In this Subsection we complete the proof of Theorem 7, by carrying out the second step in the sketch of the proof outlined at the end of Subsection 8.3. Namely, we show that, in an a.s.s.i. subset of scenarios, it is possible to assign to every pure color $C \in \mathcal{S}(\omega)$ a nonrandom number $\lambda_{C}$ such that

$$
\begin{gathered}
\lambda_{C} \in \Lambda_{i} \text { if } C \in \mathcal{S}_{i}(\omega), \\
\lambda_{C}>-\infty, \text { and } \\
\lambda_{C}(\omega)=\lambda_{\theta C}(\theta \omega),
\end{gathered}
$$

and such that, for any $C \in \mathcal{S}(\omega)$ and any $u \in W, \lim _{n \rightarrow \infty} n^{-1} \log \left\|y^{F_{C}}(n)\right\|$ exists with the initial condition $y(0)=e_{u}$, and, if we write $\lambda(C, u)$ for this limit, it can be expressed in terms of the $\lambda_{C}, C$ pure, exactly as given by parts (2i), (2ii), and (2iii) of Theorem 7 .

We proceed by induction on the levels of the inclusion partial order on colors of the spectrum, demonstrating all of the above statements as we go along. Let $\omega \in \Omega_{0}$, and consider a color $C \in \mathcal{S}_{1}(\omega)$. By equation (38), we know that $C$ is pure. We first define $\lambda_{C}$ for such $C$. To do so, consider the computation started with a basic initial condition $y(0)=e_{u}$ where $u \in C$ is chosen arbitrarily. Since $u \in C$, by equation (11) we have $u \in F_{C}$, so we have $u \in U_{1}^{*}(0, \omega)$ in the notation of Definition 28. We define

$$
\lambda_{C}=\lim _{n \rightarrow \infty} \frac{1}{n} \log \left\|A_{11}(n) A_{11}(n-1) \ldots A_{11}(1) e_{u}\right\|,
$$

where $\left(A_{11}(n), n \in \mathbf{Z}\right)$ are defined in equation (80). Note that, by Definition 29, we have $\lambda_{C} \in \Lambda_{1}$. Further, $\lambda_{C}$ is well defined, because equation (89) gives the same number, irrespective of the choice of $u \in C$. This may be argued as follows : Suppose there is some $\tilde{u} \in C, \tilde{u} \neq u$. We claim that if $n$ is large enough then $A_{11}(n) A_{11}(n-1) \ldots A_{11}(1) e_{u}$ and $A_{11}(n) A_{11}(n-1) \ldots A_{11}(1) e_{\tilde{u}}$ have the same strictly positive coordinates, these being the ones corresponding to nodes with color $C$. To see this, consider any node $v \in U_{1}^{*}(n, \omega)$. If $n$ is sufficiently large, then $C(v+n, \omega) \in \mathcal{S}(\omega)$. Suppose $C(v+n, \omega)=C$. Then $v+n$ is influenced by every node in $C$. Thus the coordinate corresponding to $v+n$ is strictly positive in both cases. This may be seen as follows : since $v+n$ is influenced by $u$, there is a path

$$
u=v_{0}, v_{1}, \ldots, v_{k}=v+n
$$


such that $\left\langle v_{i}, v_{i+1}>\in E(\omega)\right.$ for all $0 \leq i \leq k-1$. Since $v+n \in U_{1}(\omega)$, we have, by part (3) of Lemma 1, that $v_{i} \in U_{1}(\omega)$ for all $0 \leq i \leq k$. What this means is that this influence of $u$ on $v+n$ propagates through the $(1,1)$ blocks of $A(n)$, and so the coordinate of $A_{11}(n) A_{11}(n-1) \ldots A_{11}(1) e_{u}$ corresponding to the node $v$ is strictly positive. The same is true with initial condition $e_{\tilde{u}}$.

Suppose $C(v+n, \omega)=C^{\prime} \neq C$. By Definition 28 of $U_{1}^{*}(n, \omega)$ we know that $v+n \in F_{C^{\prime \prime}}$ for some $C^{\prime \prime} \in \mathcal{S}_{1}(\omega)$. Thus we must have $C^{\prime} \subseteq C^{\prime \prime}$. Since $C^{\prime \prime} \in \mathcal{S}_{1}(\omega)$, this can only hold if $C^{\prime}=C^{\prime \prime}$. This means $C^{\prime} \in \mathcal{S}_{1}(\omega)$. Now, if there is a node $u \in C$ which influences $v+n$, this means $C^{\prime} \cap C \neq \emptyset$, which, by part (4) of Proposition 4, contradicts the fact that both $C \in \mathcal{S}_{1}(\omega)$ and $C^{\prime} \in \mathcal{S}_{1}(\omega)$. This means that $v+n$ is influenced by none of the nodes in $C$. Thus the coordinate corresponding to $v+n$ is zero in both cases. That $\lambda_{C}$ as defined in equation (89) does not depend on the choice of $u \in C$ is now immediate. We have shown that equation (86) holds for $i=1$. Note that, for any nonnegative nonzero initial condition $y(0)$ supported on the nodes of $C$, we would also have

$$
\lambda_{C}=\lim _{n \rightarrow \infty} \frac{1}{n} \log \left\|A_{11}(n) A_{11}(n-1) \ldots A_{11}(1) y(0)\right\|,
$$

and we could have used equation (91) as the definition of $\lambda_{C}$.

To see that $\lambda_{C}>-\infty$, recall the definition of $\delta>0$ in Definition 19. We showed in the previous paragraph that, for any $u \in C$, if we start the computation with the initial condition $y(0)=e_{u}$, then for $n$ large enough the nonzero coordinates of $A_{11}(n) A_{11}(n-$ 1)... $A_{11}(1) y(0)$ are precisely those corresponding to the nodes in $U_{1}^{*}(n, \omega)+n$ which have color $C$. It suffices to note such nodes must exist for $n$ large enough. Indeed, in part (1) of Lemma 1 we proved that every $B$-block contains at least one node which is in $F_{C}(\omega)$. If $n$ is sufficiently large, any such node must have color $C$. This may be argued along the lines used earlier : such a node must have color $C^{\prime} \in \mathcal{S}(\omega)$, if $n$ is large enough and, since it is in $F_{C}$, we must have $C^{\prime} \subseteq C$, but since $C \in \mathcal{S}_{1}(\omega)$ this can only happen if $C^{\prime}=C$. Putting these observations together, at least one component of $A_{11}(n) A_{11}(n-1) \ldots A_{11}(1) e_{u}$ must be at least $\delta^{\lfloor n / B\rfloor}$. This verifies equation (87) for $i=1$.

We turn to demonstrating equation (88) for $i=1$. Given $u \in C$, let $y(0)=e_{u}$. We generate an initial condition $\tilde{y}(0)$ supported on $\theta C$ as follows : If $v \in \theta C$ is such that $<u, v+1>\in E(\omega)$, we give $v$ the value of $y_{v+1}(1, \omega)$, i.e. we set $\tilde{y}_{v}(0)=y_{v+1}(1, \omega)$. Further if $u-1 \in \theta C$, which by equation (12) is equivalent to $\langle u, v>\in E(\omega)$ for some $v \in F_{C}(\omega)$ with $T(v)>1$, we give $u-1$ the value 1 , i.e. we set $\tilde{y}_{u-1}(0)=1$. All other 
coordinates of $\tilde{y}(0)$ are set equal to 0 . Note that $\tilde{y}(0) \neq 0$, because if $u \in C$ with $u-1 \notin \theta C$ then by Lemma 4 there must be some node $v$ with $T(v)=0$ such that $\langle u, v+1\rangle \in E(\omega)$ and $v \in \theta C$. By equation (91) we have

$$
\lambda_{\theta C}(\theta \omega)=\lim _{n \rightarrow \infty} \frac{1}{n} \log \left\|A_{11}(n, \theta \omega) A_{11}(n-1, \theta \omega) \ldots A_{11}(1, \theta \omega) \tilde{y}(0)\right\| .
$$

Recall that $U_{1}^{*}(n, \theta \omega)=U_{1}^{*}(n+1, \omega)$, see equation (76). As argued earlier, for $n$ large enough, the strictly positive components of $A_{11}(n, \theta \omega) A_{11}(n-1, \theta \omega) \ldots A_{11}(1, \theta \omega) \tilde{y}(0)$ are precisely the nodes $w$ in $U_{1}^{*}(n, \theta \omega)$ such that $C(w+n, \theta \omega)=\theta C$, and these are in fact precisely those for which $w+n \in F_{\theta C}(\theta \omega) \cap(W+n)$. By equations (23) and (76) these are also precisely those for which $w+n+1 \in F_{C}(\omega) \cap(W+n+1)$, which, as argued earlier, are precisely those for which $C(w+n+1, \omega)=C$, and this holds precisely for those nodes in $U_{1}^{*}(n+1, \omega)$ for which $A_{11}(n) A_{11}(n-1) \ldots A_{11}(1) e_{u}$ is stricly positive. Since, by equation (82), we have $A_{11}(m, \theta \omega)=A_{11}(m+1, \omega)$ for all $m \geq n$, the truth of equation (88) for $i=1$ follows immediately.

Finally, we need to check, for any $C \in \mathcal{S}_{1}(\omega)$ and any $u \in W$, that $\lim _{n \rightarrow \infty} \frac{1}{n} \log \left\|y^{F_{C}}(n)\right\|$ with initial condition $y(0)=e_{u}$ exists, and that, if we call this limit $\lambda(C, u)$, it can be expressed as in parts (2i), (2ii), and (2iii) of Theorem 7. We just have to consider two cases. If $u \notin C$ then $y^{F_{C}}(n)=0$, so this limit exists and equals $-\infty$, i.e. $\lambda(C, u)=-\infty$, and this is in accordance with part (2i) of Theorem 7. If $u \in C$, we just saw that for $n$ sufficiently large the nonzero components of $y^{U_{1}}(n)$ are precisely those corresponding to nodes of color $C$, and that every node in $F_{C}$ has color $C$, so that, in fact, the vector on the RHS of equation (89) is just $y^{F_{C}}(n)$, so this limit exists and equals $\lambda_{C}$, i.e. $\lambda(C, u)=\lambda_{C}$, and this is in accordance with part (2ii) of Theorem 7, since $C$ is pure. The case of part (2iii) of Theorem 7 doesn't occur when $i=1$.

Let us now assume that all the claims of Theorem 7 have been demonstrated for all colors at levels $i-1$ or less, and let $C \in \mathcal{S}(\omega)$ be a color at level $i$, i.e. $C \in \mathcal{S}_{i}(\omega)$.

Suppose $C$ is not pure. Then there is no issue of defining $\lambda_{C}$. Let $u \in W$, and let $y(0)=e_{u}$. If $u \notin C$ and $v \in F_{C}(\omega)$, then $u$ does not influence $v$. This is because we must have $C(v, \omega) \subseteq C$. It follows that $\lim _{n \rightarrow \infty} \frac{1}{n} \log \left\|y^{F_{C}}(n)\right\|$ with initial condition $y(0)=e_{u}$ exists and equals $-\infty$, i.e. $\lambda(C, u)=-\infty$, and this is consistent with part (2i) of Theorem 7. Now suppose $u \in C$. Because $C$ is not pure, by Proposition 5 we have

$$
F_{C}(\omega)=\bigcup_{C^{\prime} \in \mathcal{S}_{C}} F_{C^{\prime}}(\omega)
$$


From this it follows that

$$
\max _{C^{\prime} \in \mathcal{S}_{C}}\left\|y^{F_{C^{\prime}}}(n)\right\| \leq\left\|y^{F_{C}}(n)\right\| \leq \sum_{C^{\prime} \in \mathcal{S}_{C}}\left\|y^{F_{C^{\prime}}}(n)\right\|
$$

It follows that $\lim _{n \rightarrow \infty} \frac{1}{n} \log \left\|y^{F_{C}}(n)\right\|$ with initial condition $y(0)=e_{u}$ exists and equals $\max _{C^{\prime} \in \mathcal{S}_{C}} \lim _{n \rightarrow \infty} \frac{1}{n} \log \left\|y^{F_{C^{\prime}}}(n)\right\|$. By the inductive hypothesis, this is precisely $\max _{C^{\prime} \in \mathcal{S}_{C}} \lambda\left(C^{\prime}, u\right)$, which is exactly what part (2iii) of Theorem 7 claims. The case of part (2ii) of Theorem 7 does not occur, because $C$ is not pure.

Now suppose $C \in \mathcal{S}_{i}(\omega)$ and $C$ is pure.

We first claim that there exists some $u \in C$ such that $\hat{C}(u, \omega)=C$. To see this, let $m \geq B$ and let $w \in W+m$ be a node with $C(w, \omega)=C$ (since $C$ is a color one can find such $w)$. Then, by Definition 14 we have $\hat{C}(w, \omega)=C$. List the nodes in $C$ as $\left\{v_{a}, a \in \mathcal{A}\right\}$. By the definition of color, these are precisely the nodes in $W$ that influence $w$ at scenario $\omega$. Let $C_{a}$ denote $\hat{C}\left(v_{a}, \omega\right)$. By part $(2)$ of Lemma 13 we have $\beta(-B+1, \omega)>-\infty$, where $\beta(-B+1, \omega)$ is defined in equation (49). Thus, if $k \leq \beta(-B+1, \omega)$ we have $C\left(v_{a}-k, \theta^{k} \omega\right)=\theta^{k} C_{a}$, and $C\left(w, \theta^{k} \omega\right)=\theta^{k} C$. Note that the nodes that influence $w-k$ at scenario $\theta^{k} \omega$ are precisely $\left\{v_{a}-k, a \in \mathcal{A}\right\}$. Thus we have $\theta^{k} C_{a} \subseteq \theta^{k} C$ for all $a \in \mathcal{A}$ and also $\theta^{k} C=\cup_{a \in \mathcal{A}} \theta^{k} C_{a}$. By equation (39) and parts (5) and (6) of Proposition 4 we must have $C_{a}=C$ for some $a \in \mathcal{A}$, which is what we claimed.

We next observe if $u \in C$ with $\hat{C}(u, \omega)=C$, then $u \in U_{i}^{*}(0, \omega)$. This is because, first of all $u \in C$ implies $u \in U_{i}(0, \omega)$ by equation (11) and the defintion of $U_{i}(0, \omega)$, see Definition 27 and equation (75). Secondly, if we had $u \in U_{i-1}(0, \omega)$ we would have $u \in F_{C^{\prime}}(\omega)$ for some $C^{\prime} \in \cup_{j=1}^{i-1} \mathcal{S}_{j}(\omega)$, which would mean, by equation $(23)$, that $u-k \in F_{\theta^{k} C^{\prime}}\left(\theta^{k} \omega\right)$ for all $k \in \mathbf{Z}$, but this would mean that $C\left(u-k, \theta^{k} \omega\right) \subseteq \theta^{k} C^{\prime}$ for all $k<T(u)$, which in turn means that $\hat{C}(u, \omega) \subseteq C^{\prime}$, contradicting the assumption that $\hat{C}(u, \omega)=C$.

We similarly observe that if $u \in C$ with $\hat{C}(u, \omega)=C$, then $u \in C \backslash \cup_{C^{\prime} \in \mathcal{S}_{C}^{+}} C^{\prime}$. This is argued exactly as in the preceding paragraph. Indeed, if we suppose that $u \in \cup_{C^{\prime} \in \mathcal{S}_{C}^{+}} C^{\prime}$ this would lead to the conclusion that $\hat{C}(u, \omega) \subseteq C^{\prime}$ for some $C^{\prime} \in \mathcal{S}_{C}^{+}$, contradicting the hypothesis that $\hat{C}(u, \omega)=C$.

We may now define $\lambda_{C}$. Pick $u \in C$ with $\hat{C}(u, \omega)=C$, which we just showed can be done. Define

$$
\lambda_{C}=\lim _{n \rightarrow \infty} \frac{1}{n} \log \left\|A_{i i}(n) A_{i i}(n-1) \ldots A_{i i}(1) e_{u}\right\|,
$$

where $\left(A_{i i}(n), n \in \mathbf{Z}\right)$ are defined in equation (80). By Definition 29 we have $\lambda_{C} \in \Lambda_{i}$. 
Further, $\lambda_{C}$ is well defined, because equation (93) gives the same number irrespective of the choice of $u$. To see this, suppose there is some $\tilde{u} \in C$ with $\hat{C}(\tilde{u}, \omega)=C$ and $\tilde{u} \neq u$. We claim that, if $n$ is large enough, then $A_{i i}(n) A_{i i}(n-1) \ldots A_{i i}(1) e_{u}$ and $A_{i i}(n) A_{i i}(n-1) \ldots A_{i i}(1) e_{\tilde{u}}$ have the same strictly positive components, these being the nodes in $U_{i}(n, \omega)+n$ that have color $C$. This may be shown as follows : First, let $v$ be a node in $U_{i}(n, \omega)+n$ that is influenced by $u$. If $n$ is large enough we have $C(v, \omega) \in \mathcal{S}(\omega)$, and let us denote this by $C^{\prime \prime}$. Thus $u \in C^{\prime \prime}$. Since $v \in U_{i}(n, \omega)+n$, we have $v \in F_{C^{\prime}}(\omega)$ for some $C^{\prime} \in \cup_{j=1}^{i} \mathcal{S}_{j}(\omega)$. Then $C^{\prime \prime} \subseteq C^{\prime}$, so we have $C^{\prime \prime} \in \cup_{j=1}^{i} \mathcal{S}_{j}(\omega)$. Since $u \in C^{\prime \prime}$, and $u \in C \backslash \cup_{C^{\prime} \in \mathcal{S}_{C}^{+}} C^{\prime}$, we have $C^{\prime \prime}=C$, i.e. $C(v, \omega)=C$. The same argument goes through with $u$ replaced by $\tilde{u}$. Conversely, suppose $v$ is a node in $U_{i}(n, \omega)+n$ that has color $C$. Then certainly $v$ is influenced by $u$. Also, $v \in U_{i}^{*}(n, \omega)+n$, because if $v \in U_{i-1}(n, \omega)+n$ we would have $v \in F_{C^{\prime}}(\omega)$ for some $C^{\prime} \in \cup_{j=1}^{i-1} \mathcal{S}_{j}(\omega)$, implying that $C \subseteq C^{\prime}$, a contradiction. However, in order to show that the component corresponding to $v$ in $A_{i i}(n) A_{i i}(n-1) \ldots A_{i i}(1) e_{u}$ is strictly positive we need to argue that there is a sequence of nodes $u=v_{0}, v_{1}, \ldots, v_{n}=v$, where $<v_{m}, v_{m+1}>\in E(\omega)$ and $v_{m} \in U_{i}^{*}(m, \omega)$ for each $0 \leq m \leq n-1$. We note that it suffices to show that $u \in U_{i}^{*}(0, \omega)$ in order to conclude this. This is because, by virtue of equation (77), if $u \in U_{i}^{*}(0, \omega)$ then every influence from $u$ to the node $v \in U_{i}^{*}(n, \omega)$ can propagate only through nodes in $U_{i}^{*}$, and we know that there is at least one such influence. That $u \in U_{i}^{*}(0, \omega)$ may be seen by first observing that $u \in C$ together with equation (11) implies that $u \in U_{i}(0, \omega)$, and secondly, if we had $u \in U_{i-1}(0, \omega)$ we would have $u \in F_{C^{\prime}}(\omega)$ for some $C^{\prime} \in \cup_{j=1}^{i-1} \mathcal{S}_{j}(\omega)$ and so for all $n \geq 1, u$ would influence nodes with time index at least $n$ that have color $C^{\prime}$ but we saw already that this cannot be the case. Now, a parallel argument goes through word for word with $u$ replaced by $\tilde{u}$, and this completes the proof that the definition of $\lambda_{C}$ in equation (93) does not depend on the choice of $u$. We have shown that equation (86) holds. Note that, for any nonnegative nonzero initial condition $y(0)$ supported on the nodes in $C$ which have extended color $C$, we would also have

$$
\lambda_{C}=\lim _{n \rightarrow \infty} \frac{1}{n} \log \left\|A_{i i}(n) A_{i i}(n-1) \ldots A_{i i}(1) y(0)\right\|,
$$

and we could have used this as a definition of $\lambda_{C}$.

To see that $\lambda_{C}>-\infty$, it suffices to prove that if we choose $u \in C$ with $\hat{C}(u, \omega)=C$, then for each $n$ sufficiently large we have some node $v \in U_{i}^{*}(n, \omega)$ such that $v+n$ is influenced by $u$. Indeed, we already argued that if this were true then any such influence 
would propagate through nodes in $U_{i}^{*}(m, \omega)+m, m=0,1, \ldots, n$. If we recall the definition of $\delta>0$ in Remark 19 and recall that the timewise length of any edge in $E(\omega)$ is at most $B$, it would follow that the component corresponding to $v$ in $A_{i i}(n) A_{i i}(n-1) \ldots A_{i i}(1) e_{u}$ has value at least $\delta\left\lfloor\frac{n}{B}\right\rfloor$, and this implies that $\lambda_{C}>-\infty$.

Now, we know that, for every $n \in \mathbf{Z}, W+n$ contains a node in $F_{C}(\omega)$, by part (1) of Lemma 1. We can use an argument identical to that used to prove this and show that, for every $n \geq 1, W+n$ contains a node in $F_{C}(\omega)$ that is influenced by $u$. It suffices for this to note that $u \in C$ and the influence of $u$ must propagate to infinitely many nodes of color $C$. Any such node is a node in $U_{i}^{*}(n, \omega)$ that is influenced by $u$, and this completes the proof that equation (87) holds.

We now show that equation (88) holds. Given $u \in C$ with $\hat{C}(u, \omega)=C$, consider the initial condition $y(0)=e_{u}$. We construct an initial condition for sample point $\theta \omega$ denoted by $\tilde{y}(0)$ and supported on the nodes in $\theta C$ that have extended color $\theta C$ at scenario $\theta \omega$. If $u-1 \in \theta C$, then, noting that $\hat{C}(u-1, \theta \omega)=\theta C$ by equation $(47)$, we set $\tilde{y}_{u-1}(0)=1$. If $u-1 \notin \theta C$ then Lemma 4 tells us that there is some node $v \in \theta C$ with $T(v)=0$ and $\langle u, v+1\rangle \in E(\omega)$. Since $v \in \theta C$ we have $v \in F_{\theta C}(\theta \omega)$ by equation (11), and so $\hat{C}(v, \theta \omega) \subseteq \theta C$. Since $u$ influences $v+1$ at scenario $\omega$ we have $C \subseteq \hat{C}(v+1, \omega)$, so that $\theta C \subseteq \hat{C}(v, \theta \omega)$. These two observations yield that $\hat{C}(v, \theta \omega)=C$. For any such $v$, we set $\tilde{y}_{v}(0)=y_{v+1}(1, \omega)$, with the notation being as in Definition 30 . Note that $\tilde{y}(0) \neq 0$. Further, $\tilde{y}(0)$ is supported on the nodes in $\theta C$ that have extended color $\theta C$ at scenario $\theta \omega$.

By equation (94) we have

$$
\lambda_{\theta C}(\theta \omega)=\lim _{n \rightarrow \infty} \frac{1}{n} \log \left\|A_{i i}(n, \theta \omega) A_{i i}(n-1, \theta \omega) \ldots A_{i i}(1, \theta \omega) \tilde{y}(0)\right\| .
$$

Exactly as in the case $i=1$, we see that, for $n$ large enough, subset of $U_{i}^{*}(n, \theta \omega)$ that corresponds to the strictly positive components of this expression corresponds precisely to those nodes $w$ such that $w \in U_{i}^{*}(n, \theta \omega)$ and $C(w+n, \theta \omega)=\theta C$, and these are precisely the nodes for which $w \in U_{i}^{*}(n+1, \omega)$ and $C(w+n+1, \omega)=C$. This means that the strictly positive components of $A_{i i}(n, \theta \omega) A_{i i}(n-1, \theta \omega) \ldots A_{i i}(1, \theta \omega) \tilde{y}(0)$ are precisely the same as the strictly positive components of $A_{i i}(n) A_{i i}(n-1) \ldots A_{i i}(1) e_{u}$. Since, by equation (82), we have $A_{i i}(m, \theta \omega)=A_{i i}(m+1, \omega)$ for all $m \geq n$, we have established the truth of equation (87).

Finally we need to check that, given $u \in W$, the $\operatorname{limit}_{n \rightarrow \infty} \frac{1}{n} \log \left\|y^{F_{C}}(n)\right\|$ with 
initial condition $y(0)=e_{u}$ exists, and that, if we call this limit $\lambda(C, u)$, then it can be expressed as in parts (2i), (2ii), and (2iii) of Theorem 7. If $u \notin C$, then $y^{F_{C}}(n)=0$, so this limit exists and equals $-\infty$, and this is in accordance with part (2i) of Theorem 7 .

Suppose $u \in C \backslash \cup_{C^{\prime} \in \mathcal{S}_{C}} C^{\prime}$. If $v \in F_{C}(\omega) \cap(W+n)$, then $C(v, \omega) \in \mathcal{S}(\omega)$ if $n$ is large enough, and if $C(v, \omega)=C^{\prime}$, then $C^{\prime} \subseteq C$. If $C^{\prime} \neq C$, then $C^{\prime} \in \cup_{C^{\prime} \in \mathcal{S}_{C}} C^{\prime}$, and so $v$ is not influenced by $u$. If $C^{\prime}=C$ then $v$ is influenced by $u$. The conclusion is that, with $y(0)=e_{u}$, for all $n$ large enough, the strictly positive components of $y^{F_{C}}(n)$ are precisely those corresponding to the nodes in $F_{C}(\omega) \cap(W+n)$ which have color $C$. This means that for any $u, \tilde{u} \in C \backslash \cup_{C^{\prime} \in \mathcal{S}_{C}} C^{\prime}$ there are positive constants $0<\gamma_{1} \leq \gamma_{2}<\infty$ such that

$$
\gamma_{1}\left\|\tilde{y}^{F_{c}}(n)\right\| \leq\left\|y^{F_{C}}(n)\right\| \leq \gamma_{2}\left\|\tilde{y}^{F_{c}}(n)\right\|
$$

where $\tilde{y}(0)=e_{\tilde{u}}$ and $y(0)=e_{u}$. Now, if we had $\tilde{u} \in C$ with $\hat{C}(\tilde{u}, \omega)=C$, we know from earlier arguments that

$$
\tilde{y}^{F_{c}}(n)=A_{i i}(n) \ldots A_{i i}(1) e_{\tilde{u}}
$$

and from this and equations (93) and (95) it follows that

$$
\lim _{n \rightarrow \infty} \frac{1}{n} \log \left\|y^{F_{C}}(n)\right\|=\lambda_{C} .
$$

Further, this is precisely what is claimed by part (2ii) of Theorem 7 in this case.

It remains to consider the case of $u \in \cup_{C^{\prime} \in \mathcal{S}_{C}} C^{\prime}$. Letting $y(0)=e_{u}$ as usual, we write

$$
y^{F_{C}}(n)=y_{1}^{F_{C}}(n)+y_{2}^{F_{C}}(n)
$$

where $y_{1}^{F_{C}}(n)$ is the projection of $y^{F_{C}}(n)$ setting the components corresponding to nodes that are not of color $C$ equal to 0 , and so $y_{2}^{F_{C}}(n)$ is the projection of $y^{F_{C}}(n)$ setting the components corresponding to nodes that are of color $C$ equal to 0 .

Let us first focus on $\left\|y_{2}^{F_{C}}(n)\right\|$. We first claim that, for all sufficiently large $n$, we have

$$
\left\|y_{2}^{F_{C}}(n)\right\| \geq \max _{C^{\prime} \in \mathcal{S}_{C}}\left\|y^{F_{C^{\prime}}}(n)\right\| .
$$

To see this, first observe that part (7) of Proposition 4 implies that $\cup_{C^{\prime} \in \mathcal{S}_{C}} F_{C^{\prime}} \subseteq F_{C}$. Further, if $v \in F_{C^{\prime}} \cap(W+n)$ for some $C^{\prime} \in \mathcal{S}_{C}$ and if $n$ is large enough, then $C(v, \omega)=$ $C^{\prime \prime} \in \mathcal{S}(\omega)$ where $C^{\prime \prime} \subseteq C^{\prime}$, which means in particular that $C(v, \omega) \neq C$. This proves equation (98). From equation (98) we have

$$
\varliminf_{n \rightarrow \infty} \frac{1}{n} \log \left\|y_{2}^{F_{C}}(n)\right\| \geq \max _{C^{\prime} \in \mathcal{S}_{C}} \lambda\left(C^{\prime}, u\right),
$$


by using the inductive hypothesis.

Next we observe that, if $n$ is sufficiently large and $v \in F_{C} \cap(W+n)$ with $C(v, \omega)=$ $C^{\prime} \in \mathcal{S}(\omega)$ and $C^{\prime} \neq C$, then $C^{\prime} \in \mathcal{S}_{C}$. By inductive hypothesis and Proposition 8 applied to such $C^{\prime}$ we get

$$
\varlimsup_{n \rightarrow \infty} \frac{1}{n} \log \left\|y_{2}^{F_{C}}(n)\right\| \leq \max _{C^{\prime} \in \mathcal{S}_{C}} \lambda\left(C^{\prime}, u\right),
$$

We now focus on $\left\|y_{1}^{F_{C}}(n)\right\|$. If $v \in F_{C} \cap(W+n+1)$ and $C(v, \omega)=C$ and $u \in W+n$ is such that $\langle u, v\rangle \in E(\omega)$ then $u \in F_{C} \cap(W+n)$, by part (3) of Lemma 1. If $C(u, \omega)=C$ then $u \notin U_{i-1}(n, \omega)+n$ because if we had $u \in F_{C^{\prime}}(\omega)$ for some $C^{\prime} \in \cup_{j=1}^{i-1} \mathcal{S}_{j}(\omega)$ we would have $C \subseteq C^{\prime}$, a contradiction. Thus, if $C(u, \omega)=C$ then $u \in U_{i}^{*}(n, \omega)+n$. This means that there is a matrix $A_{C}(n+1)$ such that we have

$$
y_{1}^{F_{C}}(n+1)=A_{i i}(n+1) y_{1}^{F_{C}}(n)+A_{C}(n+1) y_{2}^{F_{C}}(n)
$$

Iterating this, we obtain that for any $l \geq 1$ we have :

$y_{1}^{F_{C}}(n+l)=\sum_{k=1}^{l} A_{i i}(n+l) \ldots A_{i i}(n+k+1) A_{C}(n+k) y_{2}^{F_{C}}(n+k-1)+A_{i i}(n+l) \ldots A_{i i}(n+1) y_{1}^{F_{C}}(n)$

Let $\lambda^{0}(C, u)$ denote $\max \left\{\lambda\left(C^{\prime}, u\right): C^{\prime} \in \mathcal{S}_{C}\right\}$. For any $\epsilon>0$, from equation (100), for all $n$ sufficiently large, we have

$$
\left\|y_{2}^{F_{C}}(n)\right\| \leq \exp \left[n\left(\lambda^{0}(C, u)+\epsilon\right)\right]
$$

Pick $\tilde{u} \in C$ with $\hat{C}(\tilde{u}, \omega)=C$, and consider the initial condition $\tilde{y}(0)=e_{\tilde{u}}$. We argued earlier that, if $n$ is sufficiently large, the strictly positive components of $\tilde{y}^{F_{C}}(n)$ correspond precisely to the nodes in $F_{C} \cap(W+n)$ which have color $C$, and that $\tilde{y}^{F_{C}}(n)=$ $A_{i i}(n) \ldots A_{i i}(1) e_{\tilde{u}}$. From equation (93), for all $n$ sufficiently large, we have

$$
\exp \left[n\left(\lambda_{C}-\epsilon\right)\right] \leq\left\|\tilde{y}^{F_{C}}(n)\right\| \leq \exp \left[n\left(\lambda_{C}+\epsilon\right)\right]
$$

By Proposition 8 the convergence rate along any sequence of $C$ colored nodes is the same as the convergence rate of $\left\|\tilde{y}^{F_{C}}(n)\right\|$. Hence, if $\left\langle\tilde{y}^{F_{C}}(n)\right\rangle$ denotes the smallest strictly positive element of $\tilde{y}^{F_{C}}(n)$ we have, using equation (93), that

$$
\lim _{n \rightarrow \infty} \frac{1}{n} \log <\tilde{y}^{F_{C}}(n)>=\lambda_{C}
$$


Recall the definition of $\alpha$ in Remark 19. We have, for all sufficiently large $n$,

$$
\begin{aligned}
A_{C}(n+1) y_{2}^{F_{C}}(n) & \leq \frac{\left\|A_{C}(n+1) y_{2}^{F_{C}}(n)\right\|}{<\tilde{y}^{F_{C}}(n+1)>} \tilde{y}^{F_{C}}(n+1) \\
& \leq \frac{\alpha \exp \left[n\left(\lambda^{0}(C, u)+\epsilon\right)\right]}{\exp \left[(n+1)\left(\lambda_{C}-\epsilon\right)\right]} \tilde{y}^{F_{C}}(n+1) \\
& =\alpha \exp \left[n\left(\lambda^{0}(C, u)-\lambda_{C}+2 \epsilon\right)+\epsilon-\lambda_{C}\right] \tilde{y}^{F_{C}}(n+1),
\end{aligned}
$$

where the second step is by equations (102) and (103).

Since $u \in C$, the strictly positive entries of $y_{1}^{F_{C}}(n)$ correspond precisely to those nodes in $F_{C} \cap(W+n)$ that have color $C$. The strictly positive entries of $\tilde{y}^{F_{C}}(n)$ were also shown to correspond precisely to these nodes. Thus, there is some $0<M_{n}<\infty$ such that

$$
\frac{1}{M_{n}} \tilde{y}^{F_{C}}(n) \leq y_{1}^{F_{C}}(n) \leq M_{n} \tilde{y}^{F_{C}}(n)
$$

From equations (96), (101), (105), and (106), if $n$ is sufficiently large, we have that, for all $l \geq 1$,

$$
\begin{gathered}
y_{1}^{F_{C}}(n+l) \leq \alpha \sum_{k=1}^{l} \exp \left[(n+k-1)\left(\lambda^{0}(C, u)-\lambda_{C}+2 \epsilon\right)+\epsilon-\lambda_{C}\right] \cdot \\
{\left[A_{i i}(n+l) \ldots A_{i i}(n+k+1) \tilde{y}^{F_{C}}(n+k)\right]} \\
+M_{n}\left[A_{i i}(n+l) \ldots A_{i i}(n+1) \tilde{y}^{F_{C}}(n)\right] \\
=\left(\alpha \sum_{k=1}^{l} \exp \left[(n+k)\left(\lambda^{0}(C, u)-\lambda_{C}+2 \epsilon\right)-\lambda^{0}(C, u)-\epsilon\right]+M_{n}\right) \\
\tilde{y}^{F_{C}}(n+l) .
\end{gathered}
$$

Using equation (103) in the last expression yields

$\left\|y_{1}^{F_{C}}(n+l)\right\| \leq \exp \left[(n+l)\left(\lambda_{C}+\epsilon\right)\right]\left(\alpha \sum_{k=1}^{l} \exp \left[(n+k)\left(\lambda^{0}(C, u)-\lambda_{C}+2 \epsilon\right)-\lambda^{0}(C, u)-\epsilon\right]+M_{n}\right)$.

We now consider two cases.

First, suppose $\lambda^{0}(C, u) \geq \lambda_{C}$. Then, for any $\epsilon>0$, we have $\lambda^{0}(C, u)-\lambda_{C}+2 \epsilon>0$, and equation (108) leads to

$$
\begin{aligned}
\left\|y_{1}^{F_{C}}(n+l)\right\| & \leq \exp \left[(n+l)\left(\lambda_{C}+\epsilon\right)\right]\left(\alpha l \exp \left[(n+l)\left(\lambda^{0}(C, u)-\lambda_{C}+2 \epsilon\right)-\lambda^{0}(C, u)-\epsilon\right]+M_{n}\right) \\
& =\alpha l \exp \left[(n+l)\left(\lambda^{0}(C, u)+3 \epsilon\right)-\lambda^{0}(C, u)-\epsilon\right]+M_{n} \exp \left[(n+l)\left(\lambda_{C}+\epsilon\right)\right] .(109)
\end{aligned}
$$

Hence

$$
\varlimsup_{n \rightarrow \infty} \frac{1}{n} \log \left\|y_{1}^{F_{C}}(n+l)\right\| \leq \lambda^{0}(C, u)+3 \epsilon .
$$


Since $\epsilon>0$ is arbitrary, we have

$$
\varlimsup_{n \rightarrow \infty} \frac{1}{n} \log \left\|y_{1}^{F_{C}}(n+l)\right\| \leq \lambda^{0}(C, u) .
$$

Together with equations (97), (99), and (100) we get

$$
\lim _{n \rightarrow \infty} \frac{1}{n} \log \left\|y^{F_{C}}(n)\right\|=\lambda^{0}(C, u),
$$

in this case, and this is consistent with the claim of part (2ii) of Theorem 7.

Next, suppose $\lambda_{C}>\lambda^{0}(C, u)$. We choose $\epsilon>0$ so that

$$
\lambda_{C}-\epsilon>\lambda^{0}(C, u)+\epsilon .
$$

From equation (108) we have

$$
\left\|y_{1}^{F_{C}}(n+l)\right\| \leq \exp \left[(n+l)\left(\lambda_{C}+\epsilon\right)\right]\left(\alpha l \exp \left[n\left(\lambda^{0}(C, u)-\lambda_{C}+2 \epsilon\right)-\lambda^{0}(C, u)-\epsilon\right]+M_{n}\right)
$$

Fix $n$ (sufficiently large) and let $l \rightarrow \infty$. From equation (110) we see that

$$
\varlimsup_{l \rightarrow \infty} \frac{1}{n+l} \log \left\|y_{1}^{F_{C}}(n+l)\right\| \leq \lambda_{C}+\epsilon .
$$

By letting $\epsilon \rightarrow 0$, we have

$$
\varlimsup_{l \rightarrow \infty} \frac{1}{n+l} \log \left\|y_{1}^{F_{C}}(n+l)\right\| \leq \lambda_{C} .
$$

On the other hand, from equations (101) and (106) we have

$$
\begin{aligned}
y_{1}^{F_{C}}(n+l) & \geq \frac{1}{M_{n}} A_{i i}(n+l) \ldots A_{i i}(n+1) \tilde{y}^{F_{C}}(n) \\
& =\frac{1}{M_{n}} \tilde{y}^{F_{C}}(n+l) .
\end{aligned}
$$

Equation (112) together with equation (93) yields, on letting $l \rightarrow \infty$ for fixed $n$ sufficiently large, that

$$
\varliminf_{l \rightarrow \infty} \frac{1}{l} \log \left\|y_{1}^{F_{C}}(n+l)\right\| \geq \lambda_{C}
$$

From equations (97), (99), (100), (111), and (113) we have

$$
\lim _{n \rightarrow \infty} \frac{1}{n} \log \left\|y^{F_{C}}(n)\right\|=\lambda_{C},
$$

and, because $\lambda_{C}>\lambda^{0}(C, u)$ this is consistent with part (2ii) of Theorem 7 .

The case of part (2iii) of Theorem 7 does not arise, because $C$ is pure.

With this, we have completed the proof Theorem 7 by induction. 


\section{Removing the nonnegativity assumptions}

The results proved in the preceding sections can be used to shed some light on the evolution of asynchronous iterations with stationary ergodic interprocessor delays when the matrix being iterated and the initial condition may have negative entries. In this section, we develop such results. In the case where $A$ is a scalar $(p=1)$ every color in the spectrum corresponds to a mode of evolution of the computation, and the Oseledec subspaces can be constructed as unions of these modes. When $A$ is a non-scalar matrix $(p>1)$, we construct a family of invariant subspaces, based on the colors, and each of these can be shown to be decomposed by the Oseledec subspaces in an invariant way.

\subsection{The case of scalar $A(p=1)$.}

Since each node is influenced by exactly one other node, we have $C(u, \omega) \in\{(1,-B+$ $1), \ldots,(1,0)\}$. Let $F_{c}(\omega)$ denote the filament of the color $c$, where $c \in\{(1,-B+$ $1), \ldots,(1,0)\}$.

Remark 23 We could simplify notation by dropping the processor index, but for clarity we prefer not to.

Theorem 8 (1). For all $\omega \in \Omega$ it holds that if $u \in F_{c}(\omega)$ with $T(u) \geq 1$ then $C(u, \omega)=c$.

(2). For an a.s.s.i. subset of scenarios it holds that for any two distinct nodes $u_{1}$ and $u_{2}$ such that $u_{1}, u_{2} \in F_{c}(\omega)$, we have $u_{1} \in H\left(u_{2}, \omega\right)$ if $T\left(u_{1}\right)<T\left(u_{2}\right)$.

Proof : To prove (1) note that $u \in F_{c}(\omega)$ implies $C(u, \omega) \subseteq\{c\}$, that is, $C(u, \omega)=c$. To prove (2), let $T\left(u_{1}\right)=n_{1}$ and $T\left(u_{2}\right)=n_{2}$, so that $n_{1}<n_{2}$ by assumption. Then $T\left(u_{1}-n_{1}\right)=0$ and $T\left(u_{2}-n_{1}\right)=n_{2}-n_{1}>0$. By equation (22) we know that

$$
\left|\left\{w: C(w, \omega)=c, C\left(w-n_{1}, \theta^{n_{1}} \omega\right) \neq \theta^{n_{1}} c\right\}\right|<\infty .
$$

Since $u_{1} \in F_{c}(\omega)$ it is in the history of infinitely many nodes of color $c$ at scenario $\omega$. By equation (114) there exists some node $w_{1}$ such that

$$
C\left(w_{1}, \omega\right)=c, C\left(w_{1}-n_{1}, \theta^{n_{1}} \omega\right)=\theta^{n_{1}} c, \text { and } u_{1} \in H\left(w_{1}, \omega\right) .
$$

Similarly there exists some node $w_{2}$ such that

$$
C\left(w_{2}, \omega\right)=c, C\left(w_{2}-n_{1}, \theta^{n_{1}} \omega\right)=\theta^{n_{1}} c, \text { and } u_{2} \in H\left(w_{2}, \omega\right)
$$


From equation (115) and because $T\left(u_{1}-n_{1}\right)=0$ and $u_{1}-n_{1} \in H\left(w_{1}-n_{1}, \theta^{n_{1}} \omega\right)$, it follows that

$$
\theta^{n_{1}} c=(1,0)
$$

From equation (116) we then have $C\left(w_{2}-n_{1}, \theta^{n_{1}} \omega\right)=(1,0)=u_{1}-n_{1}$. Thus $u_{1}-n_{1} \in$ $H\left(w_{2}-n_{1}, \theta^{n_{1}} \omega\right)$. But we also have, from equation (116), that $u_{2}-n_{1} \in H\left(w_{2}-n_{1}, \theta^{n_{1}} \omega\right)$. From this, because each node can be influenced by only one other node, it follows that $u_{1}-n_{1} \in H\left(u_{2}-n_{1}, \theta^{n_{1}} \omega\right)$, i.e. that $u_{1} \in H\left(u_{2}, \omega\right)$, completing the proof.

For $-B+1 \leq m \leq 0$, let $e_{m}$ denote the initial condition with the value of node $(1, m)$ set to one and the values of all other nodes in the base set to zero. If $(1, m) \in W \backslash \mathcal{S}(\omega)$, then $(1, m)$ influences only finitely many nodes, so for scenarios in $\Omega_{0}$ we have $L\left(e_{m}, \omega\right)=$ $-\infty$. On the other hand, if $u_{1}, u_{2} \in F_{m}(\omega)$ where $(1, m) \in \mathcal{S}(\omega)$, and $T\left(u_{1}\right)<T\left(u_{2}\right)$, then by Part (2) of Theorem 8, on an a.s. subset of scenarios there exists a directed path from $u_{1}$ to $u_{2}$ in $G(\omega)$. All such paths have at least $\left\lceil\left(T\left(u_{2}\right)-T\left(u_{1}\right)\right) / B\right\rceil$ edges in them. Hence $\underline{\lim } n^{-1} \log \left\|M(n, \omega) e_{m}\right\|>-\infty$. By Theorem 1 the limit on the left exists almost surely. Therefore, in an a.s. subset of scenarios,

$$
L\left(e_{m}, \omega\right)>-\infty \text { if and only if } m \in \mathcal{S}(\omega) .
$$

Also note that, because each processor is influenced by only one other processor, $M(n, \omega) e_{i}$ is orthogonal to $M(n, \omega) e_{j}$ for all $i \neq j, n>0$, and thus,

$$
\begin{aligned}
\max \left(\left\|M(n, \omega) e_{i}\right\|,\left\|M(n, \omega) e_{j}\right\|\right) & \leq\left\|M(n, \omega)\left(e_{i}+e_{j}\right)\right\| \\
& \leq 2 \max \left(\left\|M(n, \omega) e_{i}\right\|,\left\|M(n, \omega) e_{j}\right\| .\right.
\end{aligned}
$$

Therefore, for an a.s. subset of scenarios,

$$
L\left(\sum_{i=1}^{B} \alpha_{i} e_{i}, \omega\right)=\max \left\{L\left(e_{i}, \omega\right): \alpha_{i} \neq 0\right\} .
$$

These observations lead to a description of the subspaces of OMET in terms of the colors, in the scalar case. In the statement of the following theorem $s, \delta_{k}, 1 \leq k \leq s$, and $\lambda_{k}, 1 \leq k \leq s$ are the nonrandom constants of OMET and $V_{k}(\omega), 1 \leq k \leq s$ are the random invariant subspaces of OMET.

Theorem 9 For an a.s.s.i. subset of scenarios there exists a partition $\left\{\mathcal{S}^{k}(\omega), k=\right.$ $1, \ldots, s\}$ of $W$ such that: 
(1). $\left|\mathcal{S}^{k}(\omega)\right|=\delta_{k}$ and

(2). $\mathcal{S}^{s}(\omega)=W \backslash S(\omega)$ if $\lambda_{s}=-\infty$ while $\mathcal{S}(\omega)=W$ if $\lambda_{s}>-\infty$, and

(3). $V_{k}(\omega)=\bigoplus_{i \geq k}\left(\bigoplus_{j \in \mathcal{S}^{i}(\omega)} \operatorname{spane}_{j}\right)$.

Proof : Fix some $\omega \in \Omega_{0}$. We will prove the claims assuming that $\lambda_{s}=-\infty$. The case $\lambda_{s}>-\infty$ has a very similar proof.

Define $\mathcal{S}^{k}(\omega)$ to be $\left\{i: L\left(e_{i}, \omega\right)=\lambda_{k}\right\}$. Since $L\left(e_{i}, \omega\right) \in\left\{\lambda_{1}, \ldots, \lambda_{s}\right\}$ for $i=-B+$ $1, \ldots, 0,\left\{\mathcal{S}^{k}(\omega) ; k=1, \ldots, s\right\}$ partitions $W$. We next show that

$$
\bigoplus_{i \geq k}\left(\bigoplus_{j \in \mathcal{S}^{i}(\omega)} \operatorname{span} e_{j}\right)=\left\{y: L(y, \omega) \leq \lambda_{k}\right\}
$$

If $y$ is in the set on the LHS of equation (120), then

$$
y=\sum_{i \geq k} \sum_{j \in \mathcal{S}^{i}(\omega)} \alpha_{j} e_{j}
$$

where some of the $\alpha_{j}$ 's may be zero. Then by definition of $\mathcal{S}^{k}(\omega)$ and equation (119), $L(y, \omega) \leq \lambda_{k}$.

To show the other inclusion, let $y$ be in the set on the right hand side of equation (120) and write $y=\sum_{j \in \Lambda} \alpha_{j} e_{j}$ with $\alpha_{j} \neq 0$ for all $j \in \Lambda$. Then by equation (119) we get $\Lambda \subseteq \bigcup_{i \geq k} \mathcal{S}^{i}(\omega)$ as $\lambda_{i} \leq \lambda_{k}$ for all $i \geq k$. This completes the proof of equation (120).

Now note that the set on the RHS of equation (120) is just $V_{k}(\omega)$, in the notation of Theorem 1. Therefore,

$$
\bigoplus_{i \geq k}\left(\bigoplus_{j \in \mathcal{S}^{i}(\omega)} \operatorname{span} e_{j}\right)=V_{k}(\omega)
$$

This proves (3). (2) follows from equation (118). To show (1), note that by equation (121), $\left|\mathcal{S}^{k}(\omega)\right|=\operatorname{dim} V_{k}(\omega)-\operatorname{dim} V_{k+1}(\omega)$. But this last expression is precisely $\delta_{k}$.

\subsection{The case of general non-scalar matrices}

In the non-scalar matrix case we cannot in general expect the simple picture where the Oseledec subspaces are composed of modes corresponding to the individual colors. Indeed, even for deterministic delays the situation is significantly deeper. For example, let

$$
A=\left(\begin{array}{cc}
1 & 1 \\
-2 & 4
\end{array}\right)
$$


$B=1$, and $d_{i j}=1$ for all $1 \leq i, j \leq 2$, which corresponds to a synchronous iteration. There is clearly only one color $\{(1,0),(2,0)\}$ in $\mathcal{S}(\omega)$, but there are two growth rates, namely the $\log$ of eigenvalues 2 and 3 .

There is a sense in which the colors provide a decomposition of the computation into components, despite the fact that each color may itself involve several modes of evolution, as in the example above. For instance, initial conditions whose support is disjoint from any color can never influence nodes of that color. The following theorem gives a construction of certain invariant subspaces associated to the colors and describes how these provide a finer level of decomposition of the Oseledec subspaces into invariant subspaces. In the statement of the theorem, $P_{k}(\omega)$ denotes the projection onto the subspace $\bar{V}_{k}(\omega)$ which is the orthogonal complement of $V_{k+1}(\omega)$ in $V_{k}(\omega)$, in the notation of Theorem 1, where $V_{s+1}(\omega)=\{0\}$, so that $\bar{V}_{s}(\omega)=V_{s}(\omega)$.

Theorem 10 For an a.s.s.i. subset of scenarios, for any subset $\mathcal{S} \subseteq \mathcal{S}(\omega)$,

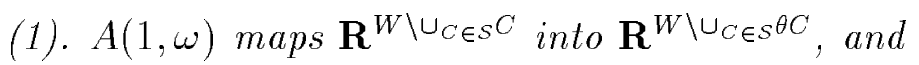

(2). For all $k=1, \ldots, s$, for which $\lambda_{k}>-\infty$,

$$
\operatorname{dim} P_{k}(\omega) \mathbf{R}^{W \backslash \cup_{C \in \mathcal{S}} C}=\operatorname{dim} P_{k}(\theta \omega) \mathbf{R}^{W \backslash \cup_{C \in \mathcal{S}^{\theta C}}}
$$

Proof : To prove (1), let $v \in \bigcup_{C \in \mathcal{S}} \theta C$. It suffices to show that the value at node $v+1$ need nor refer to the values of any of the nodes in $W \backslash \bigcup_{C \in \mathcal{S}} C$. Let $C \in \mathcal{S}$ be such that $v \in \theta C$. Recall that, by the definition of $\theta C$ and equation (10), there is some node $w$ with $T(w)>1$ and $w \in F_{C}(\omega)$ such that $\langle v+1, w>\in E(\omega)$.

Suppose $T(v)<0$. Then it suffices to show that $v+1 \notin W \backslash \bigcup_{C \in \mathcal{S}} C$. The existence of the node $w$ as in the preceding paragraph implies that $v+1 \in C$. Thus $v+1 \notin W \backslash \bigcup_{C \in \mathcal{S}} C$, as required.

Suppose $T(v)=0$. Let $u$ be any node in the base such that $<u, v+1>\in E(\omega)$, i.e. the value at node $u$ influences the value at node $v+1$. The existence of the node $w$ as in the first paragraph shows that $u$ is in the history of a node of color $C$, and since we further have $u$ directly leading to a node with time index 1 , i.e. $v+1$, we have that $u \in C$, from the definition of color in equation (5). This completes the proof of (1).

To prove (2), consider the subspaces $V_{k}(\omega) \cap \mathbf{R}^{W \backslash \cup_{C \in S^{C}}}$ for $1 \leq k \leq s$. Since by OMET $V_{k+1}(\omega) \subseteq V_{k}(\omega)$, there exists a basis $\left\{v_{1}, \ldots, v_{i_{s}}\right\}$ for $V_{s}(\omega) \cap \mathbf{R}^{W \backslash \cup_{C \in \mathcal{S}} C}$, a basis $\left\{v_{1}, \ldots, v_{i_{s}}, v_{i_{s}+1}, \ldots, v_{i_{s-1}}\right\}$ for $V_{s-1}(\omega) \cap \mathbf{R}^{W \backslash \cup_{C \in S^{C}}}$, and so on. Note that it is possible to 
have $i_{k}=i_{k+1}$. Also note that $\operatorname{dim} P_{k}(\omega) \mathbf{R}^{W \backslash \cup_{C \in S} C}=i_{k}-i_{k+1}$ and that $P_{k}(\omega) \mathbf{R}^{W \backslash \cup_{C \in S} C}$ is spanned by the vectors $P_{k}(\omega) v_{i_{k+1}+1}, \ldots, P_{k}(\omega) v_{i_{k}}$. Now fix $k$ with $\lambda_{k}>-\infty$. We first show by contradiction that,

$$
\operatorname{dim} P_{k}(\omega) \mathbf{R}^{W \backslash \cup_{C \in \mathcal{S}} C} \leq \operatorname{dim} P_{k}(\theta \omega) \mathbf{R}^{W \backslash \cup_{C \in \mathcal{S}} \theta C}
$$

Note that for $v \in V_{k}(\omega) \backslash V_{k+1}(\omega)$ and $v^{\prime}=v-P_{k}(\omega) v \in V_{k+1}(\omega)$,

$$
\begin{aligned}
P_{k}(\theta \omega) A(1, \omega) v & =P_{k}(\theta \omega) A(1, \omega)\left(P_{k}(\omega) v+v^{\prime}\right) \\
& =P_{k}(\theta \omega) A(1, \omega) P_{k}(\omega) v
\end{aligned}
$$

where the second equality is due to the fact that $A(1, \omega) V_{k+1}(\omega) \subseteq V_{k+1}(\theta \omega)$. In particular, $P_{k}(\theta \omega) A(1, \omega) v_{j}=P_{k}(\theta \omega) A(1, \omega) P_{k}(\omega) v_{j}$ for $j=i_{k+1}+1, \ldots, i_{k}$.

We now claim that the vectors $\left\{P_{k}(\theta \omega) A(1, \omega) v_{j} ; j=i_{k+1}+1, \ldots, i_{k}\right\}$ are linearly independent. If they are, then we would have

$$
\begin{aligned}
\operatorname{dim} P_{k}(\theta \omega) \mathbf{R}^{W \backslash \cup_{C \in \mathcal{S}^{\theta}} C} & \geq \operatorname{dim} P_{k}(\theta \omega) A(1, \omega) \mathbf{R}^{W \backslash \cup_{C \in \mathcal{S}} C} \\
& \geq i_{k}-i_{k+1} \\
& =\operatorname{dim} P_{k}(\omega) \mathbf{R}^{W \backslash \cup_{C \in S} C}
\end{aligned}
$$

which establishes equation (123).

Therefore, suppose that the vectors $\left\{P_{k}(\theta \omega) A(1, \omega) v_{j} ; j=i_{k+1}+1, \ldots, i_{k}\right\}$ are not linearly independent. Then, there exist constants $\alpha_{j}$, not all equal to zero, such that

$$
\sum_{j=i_{k+1}+1}^{i_{k}} \alpha_{j} P_{k}(\theta \omega) A(1, \omega) v_{j}=0
$$

This implies

$$
P_{k}(\theta \omega) A(1, \omega) \sum_{j=i_{k+1}+1}^{i_{k}} \alpha_{j} P_{k}(\omega) v_{j}=0
$$

But since $\left\{P_{k}(\omega) v_{j} ; j=i_{k+1}+1, \ldots, i_{k}\right\}$ are linearly independent in $\bar{V}_{k}(\omega)$,

$$
\sum_{j=i_{k+1}+1}^{i_{k}} \alpha_{j} P_{k}(\omega) v_{j} \in \bar{V}_{k}(\omega) \backslash\{0\} \subseteq V_{k}(\omega) \backslash V_{k+1}(\omega),
$$

so that $\sum_{j=i_{k+1}+1}^{i_{k}} \alpha_{j} P_{k}(\omega) v_{j} \notin V_{k+1}(\omega)$, whereas equation (124) implies

$$
A(1, \omega) \sum_{j=i_{k+1}+1}^{i_{k}} \alpha_{j} P_{k}(\omega) v_{j} \in V_{k+1}(\theta \omega)
$$


which in view of equation (125), contradicts OMET.

To show that equality holds a.s., let $l=|\mathcal{C}|$ and denote the set

$$
\left\{\omega: \sum_{\substack{S \subseteq \mathcal{S}(\omega) \\|\mathcal{S}|=l}} \operatorname{dim} P_{k}(\omega) \mathbf{R}^{W \backslash \cup_{C \in \mathcal{S}} C} \leq t\right\}
$$

by $Q_{l k}(t)$. Then, in view of equation $(123), \theta Q_{l k}(t) \subseteq Q_{l k}(t)$. Using an ergodic theoretic argument similar to ones used earlier, we get $P\left(Q_{l k}^{\prime}\left(t^{\prime}\right)\right)=1$ for some $t^{\prime}=t^{\prime}(l, k)$, where $P\left(Q_{l k}^{\prime}(t)\right)$ is defined like $Q_{l k}(t)$ with the inequality replaced by equality. Using equation (123) again, part (2) follows, since strict inequality in equation (123) is impossible for any $\mathcal{S}$ and any $k$.

Note that in the last part of the proof, we had to sum over all $l$-member subsets of $\mathcal{S}(\omega)$, as there is no canonical way of associating different individual $l$-member subsets of $\mathcal{S}(\omega)$ for different $\omega$ 's.

\subsection{An Example}

Here we give an example to show the need for having $\lambda_{k}>-\infty$ in the statement of Theorem 10 .

Let $A$ be a $2 \times 2$ matrix with no zero elements. Let $B=2$ and $\Omega=\left\{\omega_{0}, \omega_{1}\right\}$ with $P\left(\omega_{i}\right)=.5$ and $\theta \omega_{i}=\omega_{(i+1) \bmod 2}$. Then $(P, \theta)$ is stationary and ergodic. Let $d\left(n, \omega_{i}\right)=$ $d\left(n \bmod 2, \omega_{i}\right)$ (i.e., the delays are periodic with period 2$)$, with

$$
d\left(1, \omega_{0}\right)=\left(\begin{array}{ll}
1 & 1 \\
1 & 1
\end{array}\right) \quad \text { and } \quad d\left(2, \omega_{0}\right)=\left(\begin{array}{ll}
2 & 1 \\
1 & 2
\end{array}\right) .
$$

To make $d$ compatible with $\theta$, we let $d\left(n, \omega_{1}\right)=d\left((n+1) \bmod 2, \omega_{0}\right)$.

It is easy to show that $\mathcal{S}\left(\omega_{0}\right)=\{C\}$, where $C=\{(1,0),(2,0)\}$, and that $F_{C}\left(\omega_{0}\right)=$

$U \backslash W$. We thus get $\theta C=W$, and thus $\operatorname{dim} \mathbf{R}^{W \backslash C}=2$ while $\operatorname{dim} \mathbf{R}^{W \backslash \theta C}=0$. Upon closer inspection, we note that $\mathbf{R}^{W \backslash C}$ is in the nullspace of $A\left(1, \omega_{0}\right)$ (since its last two columns are all zero), and thus in the nullspace of $M\left(n, \omega_{0}\right), n>1$, as well. Therefore, the smallest exponent, $\lambda_{s}$, is $-\infty$, and so we get $\mathbf{R}^{W \backslash C} \subseteq V_{s}\left(\omega_{0}\right)$.

\section{Acknowledgments}

This results in this paper are from the $1995 \mathrm{PhD}$. dissertation of the first author at the School of Electrical Engineering at Cornell University, written under the supervision of 
the second author. Preparing the results for archival publication has been a task difficult to find time for. The second author would like to thank Prof. Onesimo Hernandez-Lerma of CINVESTAV, Mexico City, and Prof. John Lewis of DIAS, Dublin, for their kind hospitality in providing working environments during his sabbatical leave in Spring 1998, which enabled this task to be completed.

\section{References}

[1] Baccelli, F. and Bremaud, P. Palm Probabilities and Stationary Queues, Lecture Notes in Statistics, Vol. 41, Springer-Verlag, Berlin, 1980.

[2] Bertsekas, D. P. and Tsitsiklis, J. N., Parallel and Distributed Computation, Prentice Hall, Englewood Cliffs, New Jersey, 1989.

[3] Cohen, J. E. Kesten, H. and Newman, C. M. (editors), Random Matrices and their Application, American Mathematical Society, Providence, Rhode Island, 1986.

[4] Chazan, D. and Miranker, W. "Chaotic relaxations", Linear Algebra and its Applications, Vol. 2, 1969, pp. 199-222.

[5] Evans, D. J. (editor), Sparsity and its Applications, Cambridge University Press", Cambridge, Great Britian, 1985.

[6] Golub, G. H. and Van Loan, C. F., Matrix Computations, John Hopkins University Press, Baltimore, Washington, 1989.

[7] R. M. Gray, Probability, Random Processes, and Ergodic Properties, Springer Verlag, Berlin, New York, 1988.

[8] Kesten, H., "Oseledec's Multiplicative Ergodic Theorem: A proof", in Random Matrices and their Application, edited by Cohen, J. E. Kesten, H. and Newman, C. M., American Mathematical Society, Providence, Rhode Island, 1986.

[9] Kumar, P. R., and Varaiya, P., Stochastic Systems : Estimation, Identification, and Adaptive Control, Prentice Hall, 1986. 
[10] Oseledec, V. I., "A multiplicative ergodic theorem. Liapunov characteristic numbers for dynamical systems", Transactions of the Moscow Mathematical Society, Vol. 19, 1968, pp. 197-231. 